J. DIFFERENTIAL GEOMETRY

53 (1999) 465-516

\title{
CONSTANT HERMITIAN SCALAR CURVATURE EQUATIONS ON RULED MANIFOLDS
}

\author{
YING-JI HONG
}

In this paper we prove an existence result for Kähler metrics with constant Hermitian scalar curvature (CHSC) on ruled manifolds. This is part of a research program suggested by Simon Donaldson, [6], which is closely related to Tian's work, [16], and Yau's conjecture on EinsteinKähler metrics. The main result (Theorem A) of this paper has been announced in [9], where a partial proof has been given. We recall the statement (and assumptions) of Theorem A as follows:

[†] Assume that $\left(M: \omega_{M}\right)$ is an $m$-dimensional compact Kähler manifold with constant Hermitian scalar curvature and $\pi: E \longrightarrow M$ is a simple holomorphic vector bundle of rank $n$ over $M$ with an EinsteinHermitian metric $H_{E}$. Let $A$ denote the Einstein-Hermitian connection on $E$ induced by $H_{E}$. Let $\mathbb{P}(E)$ denote the projectivization of $E$ over $M$. Then $\mathbb{P}(E)$ is an $(m+n-1)$-dimensional complex manifold. Let $L$ be the universal line bundle over $\mathbb{P}(E)$. Then the Einstein-Hermitian metric $H_{E}$ induces a Hermitian metric $H_{L^{*}}$ on $L^{*}$ over $\mathbb{P}(E)$. Thus there is a representative

$$
\left(\frac{i}{2 \pi} F_{H_{L^{*}}}\right)=\frac{i}{2 \pi} \cdot \bar{\partial} \partial \log H_{L^{*}}
$$

of the Euler class $e\left(L^{*}\right)$ of $L^{*}$ on $\mathbb{P}(E)$ induced by the Hermitian metric $H_{L^{*}}$. Note that the representative $\left(\frac{i}{2 \pi} F_{H_{L^{*}}}\right)$ of $e\left(L^{*}\right)$ on $\mathbb{P}(E)$ induces the Fubini-Study metric on each fiber of $\check{\pi}: \mathbb{P}(E) \longrightarrow M$. Thus, for each $k \in \mathbb{N}$ large enough,

$$
\left(\frac{i}{2 \pi} F_{H_{L^{*}}}\right)+k \cdot \check{\pi}^{*} \omega_{M}
$$

Received December 21, 1998, and, in revised form, March 7, 2000. 
is a Kähler form on $\mathbb{P}(E)$.

Theorem A. Under the assumption [†] we assume further that there is no nontrivial infinitesimal deformation of Kähler forms in the Kähler class $\left[\omega_{M}\right]$ on $M$ with constant Hermitian scalar curvature. Then, for each $k \in \mathbb{N}$ large enough, there exists a Kähler form on $\mathbb{P}(E)$ in the Kähler class

$$
\left[\left(\frac{i}{2 \pi} F_{H_{L^{*}}}\right)+k \cdot \check{\pi}^{*} \omega_{M}\right]
$$

with constant Hermitian scalar curvature.

Remark. When $E$ is stable, it is known that there exists an Einstein-Hermitian connection on $E$ by the results of Donaldson ([4] and [5]), Uhlenbeck and Yau ([17]). Conversely, when $E$ is simple and admits an Einstein-Hermitian connection, it is known that $E$ must be stable $([10])$.

Instead of proving Theorem A directly, we shall actually establish the following result in this paper.

Theorem B. Under the assumption [\$] we assume further that there is no nontrivial infinitesimal deformation of Kähler forms in the Kähler class $\left[\omega_{M}\right]$ on $M$ with constant Hermitian scalar curvature. Then there exists $\gamma_{o}>0$ such that the following statement is true:

Given $\gamma>\gamma_{o}$ there exists $q_{\gamma} \in \mathbb{N}$ such that the following statement is true: Given $q>q_{\gamma}$ there exists $N_{(\gamma: q)} \in \mathbb{N}$ such that for each $N \geq N_{(\gamma: q)}$ there exists correspondingly $k_{(\gamma: q: N)} \in \mathbb{N}$ such that, for each $k \geq k_{(\gamma: q: N)}$, there exists a unique Kähler form

$$
{ }_{N} \omega_{[k]}+i \bar{\partial} \partial \psi
$$

lying in the Kähler class

$$
\left[\left(\frac{i}{2 \pi} F_{H_{L^{*}}}\right)+k \cdot \check{\pi}^{*} \omega_{M}\right]
$$

with

$$
\|\psi\|_{H^{[2 \gamma+4]}} \leq k^{-q}
$$

carrying constant Hermitian scalar curvature. Note that $\psi$ depends on the parameters $N$ and $k$.

Here ${ }_{N} \omega_{[k]}$ is defined as follows:

$$
{ }_{N} \omega_{[k]} \equiv{ }_{o} \omega_{[k]}+i \bar{\partial} \partial \phi_{0}+\sum_{\theta=1}^{N} \frac{i \bar{\partial} \partial \phi_{\theta}}{k^{\theta}}
$$


with each $\phi_{\theta}, 0 \leq \theta \leq N$, being defined via the Induction Scheme introduced in [9]. $\|\psi\|_{H^{[2 \gamma+4]}}$ is the $H^{[2 \gamma+4]}$-norm of $\psi$ with respect to the Hermitian form (metric) $\breve{\omega}$ on $\mathbb{P}(E)$ :

$$
\|\psi\|_{H^{[2 \gamma+4]}} \equiv\|\psi\|+\|\nabla \psi\|+\cdots+\left\|\nabla^{2 \gamma+4} \psi\right\|
$$

with $\|\bullet\|$ being the $L^{2}$-norm of $\bullet$ with respect to the Hermitian form (metric) $\check{\omega}$ on $\mathbb{P}(E)$.

Note that Theorem A follows from Theorem B. Actually Theorem B provides high regularity and good approximations for the solution of the constant Hermitian scalar curvature equation, depending on $k \in \mathbb{N}$ large enough, on $\mathbb{P}(E)$. Thus standard theory of elliptic partial differential equations suffices to show that the solutions found in Theorem B are smooth.

Roughly speaking, the idea behind Theorem A and Theorem B is as follows: Suppose that there is a family of Kähler metrics, depending on $k \in \mathbb{N}$, on $\mathbb{P}(E)$ with constant Hermitian scalar curvature. Then it is natural to expect that the "limit", as $k \rightarrow+\infty$, induces a Kähler metric on $M$ with constant Hermitian scalar curvature, and induces an Einstein-Hermitian metric on $E$ over $M$. However, to establish Theorem A directly, we will inevitably encounter the fact that the CHSC equation, which depends on $k \in \mathbb{N}$, will tend to become degenerate as $k \rightarrow+\infty$. On the other hand, the "degeneracy speed" of the CHSC equation is well understood. Thus we start with constructing sufficiently good approximations for the solution of the CHSC equation, depending on $k \in \mathbb{N}$, to "improve" the degeneracy of the CHSC equation. We are successful in this attempt [9]. Actually, in [9], we have established the following result:

Corollary A of [9]. Under the assumption [ $\ddagger]$ we assume further that there is no nontrivial infinitesimal deformation of Kähler forms in the Kähler class $\left[\omega_{M}\right]$ on $M$ with constant Hermitian scalar curvature. Then, for each $\gamma \geq 0$ and each large $N \in \mathbb{N}$, there exist constants $k_{(\gamma: N)} \in \mathbb{N}$ and $C_{(\gamma: N)}>0$ such that, for $k \geq k_{(\gamma: N)}$, we have

$$
\begin{aligned}
\|-(-1+m+n) \cdot c_{k} \cdot \frac{N \omega_{[k]}^{(-1+m+n)}}{(-1+m+n) !}+{ }_{N} \mathcal{E}_{[k]} & \wedge \frac{N \omega_{[k]}^{(-2+m+n)}}{(-2+m+n) !} \|_{\mathcal{C} \gamma} \\
& \leq C_{(\gamma: N)} \cdot k^{-N+m}
\end{aligned}
$$


Here ${ }_{N} \mathcal{E}_{[k]}$ is the representative of $-e(K)$ induced by the Kähler form

$$
{ }_{N} \omega_{[k]} \equiv{ }_{o} \omega_{[k]}+i \bar{\partial} \partial \phi_{0}+\sum_{\theta=1}^{N} \frac{i \bar{\partial} \partial \phi_{\theta}}{k^{\theta}}
$$

on $\mathbb{P}(E)$ with each $\phi_{\theta}$ being constructed via the Induction Scheme (introduced in [9]). $\|\bullet\|_{\mathcal{C}^{\gamma}}$ is the $\mathcal{C}^{\gamma}$-norm of $\bullet$ with respect to the Hermitian form (metric) $\breve{\omega}$ on $\mathbb{P}(E)$.

Now the next step is to show that the linearizations of the CHSC equation, depending on $k \in \mathbb{N}$, at these approximate solutions ${ }_{N} \omega_{[k]}$ are "almost uniformly invertible" (effectively invertible). With these results established, we then show that the nonlinear part of the CHSC equation, depending on $k \in \mathbb{N}$, is effectively contractive, when expanded in a small neighborhood around a sufficiently good approximate solution. Hence a family of genuine solutions, depending on $k \in \mathbb{N}$ large enough, can be found through the Contraction Mapping Theorem.

Most calculations involved in this paper are essentially direct but rather lenghty (because we are dealing with rather nonlinear 4-th order elliptic partial differential equations). We introduce certain notation in Section II to make most expressions more effective and easier to be handled with. Certain basic results, used in [9] and to be used in this paper, will be proved in the Appendix. Notation of [9] will be retained in this paper.

\section{Notation and some fundamental results}

To make this paper more self-contained we begin with some concepts and notation introduced in [9].

Note that the Einstein-Hermitian connection $A$ on $E$ defines one smooth distribution $\mathcal{H}$ of horizontal spaces on $\mathbb{P}(E)$ :

$$
T(\mathbb{P}(E))=V \oplus \mathcal{H}
$$

Here $V$ is the sub-bundle of $T(\mathbb{P}(E))$ over $\mathbb{P}(E)$ consisting of tangent vectors which are tangential to the fibers of $\check{\pi}: \mathbb{P}(E) \longrightarrow M$. Let $V^{[*]}$ denote the maximal sub-bundle of $T^{*}(\mathbb{P}(E)$ ) over $\mathbb{P}(E)$ whose action on $\mathcal{H}$ is identically zero. Then the decomposition $T(\mathbb{P}(E))=V \ominus \mathcal{H}$ of $T(\mathbb{P}(E))$ over $\mathbb{P}(E)$ induces the following corresponding decomposition

$$
T^{*}(\mathbb{P}(E))=V^{[*]} \oplus \check{\pi}^{*}\left(T^{*}(M)\right)
$$


of $T^{*}(\mathbb{P}(E)$ ) over $\mathbb{P}(E)$. Thus we have the following decomposition

$$
\wedge^{*} T^{*}(\mathbb{P}(E))=\mathcal{C}_{V} \oplus \mathcal{C}_{m} \oplus \mathcal{C}_{M}
$$

of $\wedge^{*} T^{*}(\mathbb{P}(E))$ over $\mathbb{P}(E)$. Here $\mathcal{C}_{V}=\wedge^{*} V^{[*]}$ and $\mathcal{C}_{M}=\wedge^{*} \check{\pi}^{*} T^{*}(M)$. Besides $\mathcal{C}_{m}$ is the sub-bundle of $\wedge^{*} T^{*}(\mathbb{P}(E))$ over $\mathbb{P}(E)$ consisting of the mixed components of $\wedge^{*} T^{*}(\mathbb{P}(E))$. Thus we have the following diagram

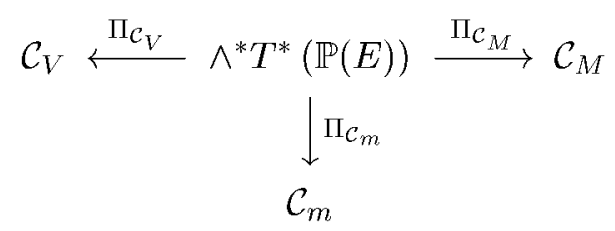

of projection maps over $\mathbb{P}(E)$ such that $i d$. $=\Pi_{\mathcal{C}_{V}} \oplus \Pi_{\mathcal{C}_{m}} \oplus \Pi_{\mathcal{C}_{M}}$ on $\wedge^{*} T^{*}(\mathbb{P}(E)$ ). Let $\Gamma(\mathbb{P}(E): \mathbb{R})$ denote the space of smooth $\mathbb{R}$-valued functions on $\mathbb{P}(E)$. We introduce one Hermitian form (metric) $\check{\omega}$ on $\mathbb{P}(E)$ by setting

$$
\check{\omega}=\Pi_{\mathcal{C}_{V}}\left(\frac{i}{2 \pi} F_{H_{L^{*}}}\right)+\check{\pi}^{*} \omega_{M} .
$$

Note that the derivation operator

$$
d: \Gamma(\mathbb{P}(E): \mathbb{R}) \longrightarrow \Gamma\left(\mathbb{P}(E): T^{*}(\mathbb{P}(E)) \otimes \mathbb{R}\right)
$$

can be expressed as

$$
d=d_{V}+d_{M}
$$

in which

$$
d_{V}: \Gamma(\mathbb{P}(E): \mathbb{R}) \longrightarrow \Gamma\left(\mathbb{P}(E): \mathbb{R} \otimes V^{[*]}\right)
$$

and

$$
d_{M}: \Gamma(\mathbb{P}(E): \mathbb{R}) \longrightarrow \Gamma\left(\mathbb{P}(E): \mathbb{R} \otimes \check{\pi}^{*}\left(T^{*}(M)\right)\right) .
$$

Let $d_{V}^{*}$ and $d_{M}^{*}$ be respectively the adjoint operators of $d_{V}$ and $d_{M}$ with respect to the Hermitian form (metric) $\breve{\omega}$. Then we have

$$
\Delta=d^{*} \circ d=\Delta_{V}+\Delta_{M}
$$

in which $\Delta_{V} \equiv d_{V}^{*} \circ d_{V}$ and $\Delta_{M} \equiv d_{M}^{*} \circ d_{M}$. Similarly we have $\bar{\partial}=$ $\bar{\partial}_{V}+\bar{\partial}_{M}$ and $\partial=\partial_{V}+\partial_{M}$. Let $\Lambda_{V}$ and $\Lambda_{M}$ be respectively the adjoint operators of

$$
\bullet \longmapsto \Pi_{\mathcal{C}_{V}}\left(\frac{i}{2 \pi} F_{H_{L^{*}}}\right) \wedge \bullet
$$

and

$$
\text { - } \longmapsto \check{\pi}^{*} \omega_{M} \wedge \bullet
$$


on $\mathbb{P}(E)$ with respect to the Hermitian form (metric) $\breve{\omega}$. We will use the symbols $(\bar{\partial} \partial)_{V}$ and $(\bar{\partial} \partial)_{M}$ to denote respectively $\Pi_{\mathcal{C}_{V}} \circ(\bar{\partial} \partial)$ and $\Pi_{\mathcal{C}_{M}} \circ(\bar{\partial} \partial)$ :

$$
(\bar{\partial} \partial)_{V}=\Pi_{\mathcal{C}_{V}} \circ(\bar{\partial} \partial) \text { and }(\bar{\partial} \partial)_{M}=\Pi_{\mathcal{C}_{M}} \circ(\bar{\partial} \partial) .
$$

Similarly we use the symbol $(\bar{\partial} \partial)_{m}$ to denote $\Pi_{\mathcal{C}_{m}} \circ(\bar{\partial} \partial)$ :

$$
(\bar{\partial} \partial)_{m}=\Pi_{\mathcal{C}_{m}} \circ(\bar{\partial} \partial) \text {. }
$$

Then we have the following results.

Proposition I.A. Given $f \in \Gamma(\mathbb{P}(E): \mathbb{R})$ we have the following equalities

$$
i \cdot \Lambda_{V} \circ(\bar{\partial} \partial)_{V} f=\frac{\Delta_{V} f}{2}
$$

and

$$
i \cdot \Lambda_{M} \circ(\bar{\partial} \partial)_{M} f=\frac{\Delta_{M} f}{2}
$$

Proposition I.B. $\Delta_{M} \circ \Delta_{V}=\Delta_{V} \circ \Delta_{M}$.

These results will be proved in the Appendix of this article.

Since the restriction of $\left(\frac{i}{2 \pi} F_{H_{L^{*}}}\right)$ on each fiber of $\check{\pi}: \mathbb{P}(E) \longrightarrow M$ is simply the Fubini-Study Kähler form, there is a well-defined vector bundle $W$ over $M$ whose fiber over each point $z \in M$ is the eigenspace of the lowest non-zero eigen-value of the (Fubini-Study) Laplacian on $\breve{\pi}^{-1}(z)$. On the other hand, integration along the fibers maps one smooth function on $\mathbb{P}(E)$ onto one smooth function on $M$. Thus for each smooth $\mathbb{R}$-valued function $f \in \Gamma(\mathbb{P}(E): \mathbb{R})$ on $\mathbb{P}(E)$ we have

$$
f=\hat{\sigma}(f) \oplus \sigma(f) \oplus \tilde{\sigma}(f),
$$

in which $(\hat{\sigma}(f): \sigma(f)) \in \Gamma(M: \mathbb{R}) \oplus \Gamma(M: W)$ and the restriction of $\tilde{\sigma}(f)$ on each fiber $\check{\pi}^{-1}(z)$ of $\check{\pi}: \mathbb{P}(E) \longrightarrow M$ is orthogonal to both the space of constant functions on $\check{\pi}^{-1}(z)$ and the space $W_{z}$. Here $\Gamma(M: W)$ is the space of smooth sections of $W$ over $M$, and $W_{z}$ is the fiber of $W$ over $z \in M$. Note that the smooth distribution $\mathcal{H}$ defines one connection $\nabla^{\mathcal{H}}$ on the fiber bundle $\check{\pi}: \mathbb{P}(E) \longrightarrow M$ preserving the bundle $W$ over $M$.

Now we state an important result about a special kind of quadratic combination of traceless Hermitian quadratic forms. 
Proposition A of [9]. Assume that $\mathbb{C}^{n}$ and $\mathbb{P}\left(\mathbb{C}^{n}\right)$ are respectively endowed with the standard Hermitian metric $H=\sum \delta_{\alpha \beta} \cdot w_{\alpha} \cdot \bar{w}_{\beta}$ on $\mathbb{C}^{n}$ and the Fubini-Study metric on $\mathbb{P}\left(\mathbb{C}^{n}\right)$. Then for any traceless Hermitian quadratic form $q=\sum q_{\alpha \beta} \cdot w_{\alpha} \cdot \bar{w}_{\beta}$ on $\mathbb{C}^{n}$ (with $\sum q_{\gamma \gamma}=0$ ) the function

$$
\begin{array}{r}
\frac{-4 \pi \cdot\left(\sum \delta_{\alpha \beta} \cdot w_{\alpha} \cdot \bar{w}_{\beta}\right) \cdot \sum q_{\alpha \gamma} \cdot q_{\gamma \beta} \cdot w_{\alpha} \cdot \bar{w}_{\beta}}{\left(\sum \delta_{\alpha \beta} \cdot w_{\alpha} \cdot \bar{w}_{\beta}\right)^{2}} \\
+\frac{(4 \pi+2 \pi n) \cdot\left(\sum q_{\alpha \beta} \cdot w_{\alpha} \cdot \bar{w}_{\beta}\right)^{2}}{\left(\sum \delta_{\alpha \beta} \cdot w_{\alpha} \cdot \bar{w}_{\beta}\right)^{2}}
\end{array}
$$

on $\mathbb{P}\left(\mathbb{C}^{n}\right)$ is orthogonal to the eigen-space of the lowest non-zero eigenvalue of the (Fubini-Study) Laplacian on $\mathbb{P}\left(\mathbb{C}^{n}\right)$.

This result can be expressed equivalently in the following way:

Let $\omega_{F-S}=-\frac{i}{2 \pi} \bar{\partial} \partial \log H$ be the Fubini-Study Kähler form on $\mathbb{P}\left(\mathbb{C}^{n}\right)$. We define the function $Q\left(\frac{q}{H}: \frac{q}{H}\right)$ on $\mathbb{P}\left(\mathbb{C}^{n}\right)$ as follows:

$$
Q\left(\frac{q}{H}: \frac{q}{H}\right) \cdot \frac{\omega_{F-S}^{(-1+n)}}{(-1+n) !}=i \bar{\partial} \partial\left(\frac{q}{H}\right) \wedge i \bar{\partial} \partial\left(\frac{q}{H}\right) \wedge \frac{\omega_{F-S}^{(-3+n)}}{(-3+n) !} .
$$

Then the function

$$
-\left(2 \pi \cdot n \cdot \frac{q}{H}\right) \cdot\left(2 \pi \cdot n \cdot \frac{q}{H}\right)+Q\left(\frac{q}{H}: \frac{q}{H}\right)
$$

on $\mathbb{P}\left(\mathbb{C}^{n}\right)$ is orthogonal to the eigen-space of the lowest non-zero eigenvalue of the (Fubini-Study) Laplacian on $\mathbb{P}\left(\mathbb{C}^{n}\right)$.

Actually for any traceless Hermitian quadratic form $q=\sum q_{\alpha \beta} \cdot w_{\alpha}$. $\bar{w}_{\beta}$ on $\mathbb{C}^{n}$ we have

$$
\begin{array}{r}
-\left(2 \pi \cdot n \cdot \frac{q}{H}\right) \cdot\left(2 \pi \cdot n \cdot \frac{q}{H}\right)+Q\left(\frac{q}{H}: \frac{q}{H}\right)+4 \pi^{2} \cdot \sum q_{\alpha \beta} \cdot q_{\beta \alpha} \\
=-\pi \cdot\left(-4 \pi n \cdot i d .+\Delta_{F-S}\right)\left(\frac{q}{H} \cdot \frac{q}{H}\right) .
\end{array}
$$

Here $\Delta_{F-S}$ is the Laplacian operator induced by the Fubini-Study Kähler form $\omega_{F-S}$ on $\mathbb{P}\left(\mathbb{C}^{n}\right)$. Note that the constant function $4 \pi^{2} \cdot \sum q_{\alpha \beta} \cdot q_{\beta \alpha}$ on $\mathbb{P}\left(\mathbb{C}^{n}\right)$ associated with $q=\sum q_{\alpha \beta} \cdot w_{\alpha} \cdot \bar{w}_{\beta}$ only depends on the unitary group orbit of $q$.

Remarks. Some remarks about this Proposition now follow.

- Note that the eigenspace of the lowest non-zero eigen-value $4 \pi n$ of the (Fubini-Study) Laplacian on $\mathbb{P}\left(\mathbb{C}^{n}\right)$ simply consists of the quotients of traceless Hermitian quadratic forms on $\mathbb{C}^{n}$ by the Hermitian metric $H=\sum \delta_{\alpha \beta} \cdot w_{\alpha} \cdot \bar{w}_{\beta}$ on $\mathbb{C}^{n}$. It is well-known in Kähler geometry that 
this eigenspace represents the tangent space at $H=\sum \delta_{\alpha \beta} \cdot w_{\alpha} \cdot \bar{w}_{\beta}$ of the moduli space of Einstein-Kähler metrics on $\mathbb{P}\left(\mathbb{C}^{n}\right)$.

- Though in this Proposition we only consider a special kind of quadratic combination of a single traceless Hermitian quadratic form $q$ on $\mathbb{C}^{n}$ it is obvious that similar result holds for the same kind of quadratic combination of two possibly different traceless Hermitian quadratic forms on $\mathbb{C}^{n}$. We can infer this easily by considering the infinitesimal deformations of $q$. This simple corollary will be referred to as the infinitesimal version of the Proposition above.

- We now indicate the power of the above Proposition (and its infinitesimal version) briefly. When dealing with the CHSC equation, depending on $k \in \mathbb{N}$, on $\mathbb{P}(E)$ we often encounter some special kind of quadratic combination of sections of $W$ over $M$. At first sight it may seem that such kind of nonlinear combination of sections of $W$ contributes nontrivial sections of $W$ over $M$. However, in reality, such kind of (nonlinear) quadratic combination of sections of $W$ over $M$ only contributes the zero section of $W$ over $M$ as indicated by the Proposition above. It is this interesting phenomenon which makes the Induction Scheme developed in [9] possible.

Now we set

$$
\Omega_{M} \equiv \frac{\omega_{M}^{m}}{m !}
$$

and

$$
\Omega \equiv \frac{\check{\omega}^{(-1+m+n)}}{(-1+m+n) !}=\frac{\left(\Pi_{\mathcal{C}_{V}}\left(\frac{i}{2 \pi} F_{H_{L^{*}}}\right)\right)^{(-1+n)}}{(-1+n) !} \wedge \frac{\check{\pi}^{*} \omega_{M}^{m}}{m !} .
$$

Let $\wedge^{\max } T^{*}(\mathbb{P}(E))$ denote the trivial bundle over $\mathbb{P}(E)$ consisting of differential forms on $\mathbb{P}(E)$ of maximal degree. Then we can identify functions on $\mathbb{P}(E)$ with sections of $\wedge^{\max } T^{*}(\mathbb{P}(E)$ ) over $\mathbb{P}(E)$ through the following map

$$
f \longmapsto f \cdot \Omega \text {. }
$$

Similarly we can identify functions on $M$ with sections of $\wedge^{\max } T^{*}(M)$ over $M$ through the map

$$
f \longmapsto f \cdot \Omega_{M}
$$

Here $\wedge^{\max } T^{*}(M)$ is the trivial bundle over $M$ consisting of differential forms on $M$ of maximal degree. Besides functions on $\mathbb{P}(E)$ which are constant along the fibers of $\check{\pi}: \mathbb{P}(E) \longrightarrow M$ will be identified with functions on $M$. 
We now recall the main result of [9] briefly. Let

$$
{ }_{o} \omega_{[k]} \equiv\left(\frac{i}{2 \pi} F_{H_{L^{*}}}\right)+k \cdot \check{\pi}^{*} \omega_{M}
$$

and ${ }_{o} H_{[k]}$ be the Kähler metric on $\mathbb{P}(E)$ induced by the Kähler form ${ }_{o} \omega_{[k]}$. Suppose that, for each $k \in \mathbb{N}$ large enough, $\omega_{[k]}$ is the (possible) Kähler form (lying in the Kähler class $\left[{ }_{o} \omega_{[k]}\right]$ ) on $\mathbb{P}(E)$ which carries CHSC. Let $K$ be the canonical line bundle of $\mathbb{P}(E)$, and $\mathcal{E}_{[k]}$ the representative of $-e(K)=e\left(K^{*}\right)$ induced by $\omega_{[k]}$ on $\mathbb{P}(E)$. Then the CHSC eqaution for $\omega_{[k]}$ is as follows:

$$
\mathcal{E}_{[k]} \wedge \frac{\omega_{[k]}^{(-2+m+n)}}{(-2+m+n) !}=(-1+m+n) \cdot c_{k} \cdot \frac{\omega_{[k]}^{(-1+m+n)}}{(-1+m+n) !} .
$$

Here the constant $c_{k}$, depending on $k \in \mathbb{N}$, is determined topologically by the following identity:

$$
\int_{\mathbb{P}(E)} \mathcal{E}_{[k]} \wedge \frac{\omega_{[k]}^{(-2+m+n)}}{(-2+m+n) !}=\int_{\mathbb{P}(E)}(-1+m+n) \cdot c_{k} \cdot \frac{\omega_{[k]}^{(-1+m+n)}}{(-1+m+n) !}
$$

Let $K_{M}$ denote the canonical line bundle of $M$. It is easy to see that $c_{k}$ admits the following asymptotic expansion:

$$
c_{k}=\frac{(-1+n) \cdot n}{(-1+m+n)}+\frac{1}{k} \cdot \frac{m}{(-1+m+n)} \cdot \frac{-e\left(K_{M}\right)}{\omega_{M}}+O\left(k^{-2}\right) .
$$

Here the constant $\frac{-e\left(K_{M}\right)}{\omega_{M}}=-\frac{e\left(K_{M}\right)}{\omega_{M}}$ is defined through the following equality

$$
\int_{M} \frac{-e\left(K_{M}\right)}{\omega_{M}} \cdot \frac{\omega_{M}^{m}}{m !}=\int_{M} \frac{-e\left(K_{M}\right)}{m} \wedge \frac{\omega_{M}^{(-1+m)}}{(-1+m) !}
$$

Let $F_{H_{E}}$ be the curvature form on $M$ induced by the Einstein-Hermitian metric on $E$. Note that the Einstein-Hermitian condition implies that

$$
\begin{aligned}
& \left(\frac{i}{2 \pi} F_{H_{L^{*}}}\right)^{n} \wedge \omega_{M}^{(-1+m)} \\
& \quad+\left(\frac{i}{2 \pi} F_{H_{L^{*}}}\right)^{(-1+n)} \wedge \omega_{M}^{(-1+m)} \wedge \check{\pi}^{*} \operatorname{trace}\left(\frac{i}{2 \pi} F_{H_{E}}\right) \\
& \quad=0
\end{aligned}
$$


Let $H_{M}$ be the Kähler metric on $M$ induced by the Kähler form $\omega_{M}$. Since $H_{E}$ is Einstein-Hermitian it can be checked directly (using the Special Coordinate System introduced in the Appendix) that

$$
\begin{aligned}
\frac{i}{2 \pi} \bar{\partial} \partial \log \operatorname{det}{ }_{o} H_{[k]}= & n \cdot\left(\frac{i}{2 \pi} F_{H_{L^{*}}}\right)+\check{\pi}^{*} \operatorname{trace}\left(\frac{i}{2 \pi} F_{H_{E}}\right) \\
& +\check{\pi}^{*} \operatorname{trace}\left(\frac{i}{2 \pi} F_{H_{M}}\right)+O_{\mathcal{C}_{M}}\left(k^{-1}\right) \\
& +O_{\mathcal{C}_{m}}\left(k^{-2}\right)+O_{\mathcal{C}_{V}}\left(k^{-2}\right) .
\end{aligned}
$$

Here $F_{H_{M}}$ is the curvature form of the holomorphic tangent bundle of $M$ induced by the Kähler metric $H_{M}$ on $M$. In [9] it is shown that the asymptotic expansion

$$
{ }_{o} \omega_{[k]}+i \bar{\partial} \partial \phi_{0}+\sum_{\theta=1}^{\infty} \frac{i \bar{\partial} \partial \phi_{\theta}}{k^{\theta}}
$$

of the (possible) solutions, depending on $k \in \mathbb{N}$ large enough, of the CHSC equation on $\mathbb{P}(E)$ can be uniquely determined (solved) by choosing the $\mathbb{R}$-valued functions $\phi_{0}$ and $\phi_{1}$ on $\mathbb{P}(E)$ satisfying the following conditions:

$$
\sigma\left(\phi_{0}\right)=0=\tilde{\sigma}\left(\phi_{0}\right) \text { and } \tilde{\sigma}\left(\phi_{1}\right)=0
$$

so that $\phi_{0}=\hat{\sigma}\left(\phi_{0}\right)$ and $\phi_{1}=\hat{\sigma}\left(\phi_{1}\right) \oplus \sigma\left(\phi_{1}\right)$. Here each $\phi_{\theta}$ is a smooth $\mathbb{R}$-valued function on $\mathbb{P}(E)$. Because this asymptotic expansion is inferred through expanding the CHSC equation for $\omega_{[k]}$ in the powers of $\frac{1}{k}$ Corollary $\mathrm{A}$ of [9] then follows directly from the existence result of the asymptotic expansion of the (possible) solutions, depending on $k \in \mathbb{N}$ large enough, of the CHSC equation on $\mathbb{P}(E)$. Because of this we will call, for each $N \in \mathbb{N}$,

$$
{ }_{N} \omega_{[k]} \equiv{ }_{o} \omega_{[k]}+i \bar{\partial} \partial \phi_{0}+\sum_{\theta=1}^{N} \frac{i \bar{\partial} \partial \phi_{\theta}}{k^{\theta}}
$$

an approximate solution for the CHSC equation depending on $k \in \mathbb{N}$. We will use ${ }_{N} H_{[k]}$ to indicate the Kähler metric on $\mathbb{P}(E)$ induced by the Kähler form ${ }_{N} \omega_{[k]}$.

Now we introduce certain linear operators which will appear naturally in the next section. We define the operators $Q_{V}\left(\bullet: \phi_{1}\right)$ and $Q_{m}\left(\bullet: \phi_{1}\right)$ on $\mathbb{P}(E)$ by setting

$$
\begin{aligned}
& Q_{V}\left(\psi: \phi_{1}\right) \cdot \frac{e\left(L^{*}\right)^{(-1+n)}}{(-1+n) !} \wedge \omega_{M}^{m} \\
& \quad=i(\bar{\partial} \partial)_{V} \psi \wedge i(\bar{\partial} \partial)_{V} \phi_{1} \wedge \frac{e\left(L^{*}\right)^{(-3+n)}}{(-3+n) !} \wedge \omega_{M}^{m}
\end{aligned}
$$


and

$$
\begin{aligned}
Q_{m} & \left(\psi: \phi_{1}\right) \cdot \frac{e\left(L^{*}\right)^{(-1+n)} \wedge \omega_{M}^{m}}{(-1+n) ! \cdot m !} \\
& =i(\bar{\partial} \partial)_{m} \psi \wedge i(\bar{\partial} \partial)_{m} \phi_{1} \wedge \frac{e\left(L^{*}\right)^{(-2+n)} \wedge \omega_{M}^{(-1+m)}}{(-2+n) ! \cdot(-1+m) !}
\end{aligned}
$$

$\forall \psi \in \Gamma(\mathbb{P}(E): \mathbb{R})$. By the infinitesimal version of Proposition A of [9] we have

$$
\sigma\left(-\frac{\Delta_{V} \phi_{1}}{2} \cdot \frac{\Delta_{V} \sigma(\psi)}{2}+Q_{V}\left(\sigma(\psi): \phi_{1}\right)\right)=0
$$

$\forall \psi \in \Gamma(\mathbb{P}(E): \mathbb{R})$. Besides we define the operator $Q_{M}(\bullet)$ on $\mathbb{P}(E)$ as follows:

$$
\begin{aligned}
& Q_{M}(\psi) \cdot e\left(L^{*}\right)^{(-1+n)} \wedge \frac{\omega_{M}^{m}}{m !} \\
& \quad=e\left(L^{*}\right)^{(-1+n)} \wedge \frac{\omega_{M}^{(-2+m)}}{(-2+m) !} \wedge i(\bar{\partial} \partial)_{M} \psi \wedge\left(i(\bar{\partial} \partial)_{M} \phi_{0}+\Pi_{\mathcal{C}_{M}} e\left(L^{*}\right)\right) .
\end{aligned}
$$

Then we have the following simple result.

Lemma I.A. Assume that $f \in \Gamma(M: \mathbb{R})$. We define the smooth function $\breve{Q}_{M}(f)$ on $\mathbb{P}(E)$ as follows:

$$
\begin{aligned}
\check{Q}_{M}(f) \cdot \frac{e\left(L^{*}\right)^{(-1+n)} \wedge \omega_{M}^{m}}{(-1+n) ! \cdot m !} & =\frac{e\left(L^{*}\right)^{(-1+n)} \wedge \omega_{M}^{(-2+m)}}{(-1+n) ! \cdot(-2+m) !} \wedge i(\bar{\partial} \partial)_{M} f \wedge \\
& \left(n \cdot \Pi_{\mathcal{C}_{M}} e\left(L^{*}\right)+\check{\pi}^{*} \operatorname{trace}\left(\frac{i}{2 \pi} F_{H_{E}}\right)\right) .
\end{aligned}
$$

Then for $\psi \in \Gamma(\mathbb{P}(E): \mathbb{R})$ we have

$$
\frac{\Delta_{V} Q_{M}(\hat{\sigma}(\psi))}{4 \pi}=\check{Q}_{M}(\hat{\sigma}(\psi)) .
$$

This result can be expressed equivalently as follows:

$$
\begin{array}{r}
\frac{\Delta_{V} Q_{M}(\hat{\sigma}(\psi))}{4 \pi} \cdot e\left(L^{*}\right)^{(-1+n)} \wedge \frac{\omega_{M}^{m}}{m !}=\frac{\omega_{M}^{(-2+m)}}{(-2+m) !} \wedge i(\bar{\partial} \partial)_{M} \hat{\sigma}(\psi) \wedge \\
\left(e\left(L^{*}\right)^{n}+e\left(L^{*}\right)^{(-1+n)} \wedge \check{\pi}^{*} \operatorname{trace}\left(\frac{i}{2 \pi} F_{H_{E}}\right)\right)
\end{array}
$$

$\forall \psi \in \Gamma(\mathbb{P}(E): \mathbb{R})$

Besides for $\psi \in \Gamma(\mathbb{P}(E): \mathbb{R})$ we have

$$
\Delta_{V} Q_{M}(\hat{\sigma}(\psi)) \in \Gamma(M: W) .
$$


Proof of this result can be found in the Appendix of this article. Lemma I.A will only be used in the next section.

Now we discuss the relation between the invertibility of $\Delta_{M}$ on $\Gamma(M: W)$ and the simplicity of $E$ over $M$. Let $d_{\underline{A}}$ denote the exterior derivation operator acting on smooth $\operatorname{Hom}(E: \bar{E})$-valued differential forms on $M$ induced by the Einstein-Hermitian connection $A$ on $E$. Let $d_{\underline{A}}^{*}$ denote the adjoint of $d_{\underline{A}}$. Then by the Kähler identities (for sections of $\operatorname{Hom}(E: E)$ over $M)$ we have

$$
\partial_{\underline{A}}^{*} \circ \partial_{\underline{A}}=\frac{d_{\underline{A}}^{*} \circ d_{\underline{A}}}{2}=\bar{\partial}_{\underline{A}}^{*} \circ \bar{\partial}_{\underline{A}},
$$

because the $\omega_{M}$-component of the curvature form of $\operatorname{Hom}(E: E)$ is zero by the Einstein-Hermitian condition. Here $\partial_{\underline{A}}$ and $\bar{\partial}_{\underline{A}}$ are respectively the $\partial$-component and the $\bar{\partial}$-component of $d_{\underline{A}}$ :

$$
d_{\underline{A}}=\partial_{\underline{A}}+\bar{\partial}_{\underline{A}} \text {. }
$$

Thus $E$ is simple if and only if the kernel of $d_{\underline{A}}^{*}$ od $d_{\underline{A}}$ on $\Gamma(M: \operatorname{Hom}(E: E))$ only consists of transformations of $E$ of the following form

$$
c \cdot i d
$$

where $c \in \mathbb{C}$.

Now we discuss the action of $\Delta_{M}$ on $\Gamma(M: W)$. Let $H$ denote the standard Hermitian metric on $\mathbb{C}^{n}$. Note that the space of quotients of traceless Hermitian quadratic forms on $\mathbb{C}^{n}$ by $H$ can be naturally identified with the space

$$
i \cdot \mathfrak{s u}\left(\mathbb{C}^{n}: H\right)
$$

of traceless linear Hermitian transformations of $\mathbb{C}^{n}$. Actually the standard Hermitian metric $H$ on $\mathbb{C}^{n}$ is preserved by $S U\left(\mathbb{C}^{n}: H\right)$, and we have the following Lie algebra decomposition

$$
\mathfrak{s l}\left(\mathbb{C}^{n}\right)=\mathfrak{s u}\left(\mathbb{C}^{n}: H\right) \oplus i \cdot \mathfrak{s u}\left(\mathbb{C}^{n}: H\right)
$$

of $\mathfrak{s l}\left(\mathbb{C}^{n}\right)$. Since the Lie algebra of $P G L\left(\mathbb{C}^{n}\right)$ is naturally isomorphic to $\mathfrak{s l}\left(\mathbb{C}^{n}\right)$, the above Lie algebra decomposition is thence valid for the Lie algebra of $P G L\left(\mathbb{C}^{n}\right)$. Now we note that for $s \in \Gamma(M: W)$

$$
\Phi \circ \Delta_{M} S=d_{\underline{A}}^{*} \circ d_{\underline{A}} \Phi s .
$$


Here $\Phi: W \longrightarrow i \cdot \mathfrak{s u}\left(E: H_{E}\right)$ is the natural isomorphism between the bundles $W$ and $i \cdot \mathfrak{s u}\left(E: H_{E}\right)$ over $M$ as described above. Thus $E$ is simple if and only if the kernel of $\Delta_{M}$ on $\Gamma(M: W)$ is trivial.

Now we introduce Sobolev spaces. Assume that

$$
\text { - } \in \Gamma\left(\mathbb{P}(E): \otimes^{\theta} T^{*}(\mathbb{P}(E))\right) \text {. }
$$

Here $\theta \in \mathbb{Z}$ and $\theta \geq 0$. We will use the symbol $\|\bullet\|$ to indicate the $L^{2}$ norm of $\bullet$ with respect to the Hermitian form (metric) $\check{\omega}$ on $\mathbb{P}(E)$. Besides we use $\nabla$ to indicate the covariant derivation operator

$$
\begin{aligned}
\nabla: \bigoplus_{\theta} \Gamma\left(\mathbb{P}(E): \otimes^{\theta} T^{*}(\mathbb{P}(E))\right) \\
\quad \longrightarrow \bigoplus_{\theta} \Gamma\left(\mathbb{P}(E):\left(\otimes^{\theta} T^{*}(\mathbb{P}(E))\right) \otimes T^{*}(\mathbb{P}(E))\right)
\end{aligned}
$$

so that for $s \in \bigoplus_{\theta} \Gamma\left(\mathbb{P}(E): \otimes^{\theta} T^{*}(\mathbb{P}(E))\right)$ we have

$$
(\nabla s) x=\nabla_{x} s
$$

$\forall x \in T(\mathbb{P}(E))$. Assume that $\psi \in \Gamma(\mathbb{P}(E): \mathbb{R})$. Given $\gamma \in \mathbb{N}$ we define the $H^{[\gamma]}$-norm of $\psi$ as follows:

$$
\|\psi\|_{H[\gamma]} \equiv\|\psi\|+\|\nabla \psi\|+\cdots+\left\|\nabla^{\gamma} \psi\right\| .
$$

Thus $H^{[\gamma]}$ is the Sobolev space obtained by completing the space $\Gamma(\mathbb{P}(E): \mathbb{R})$ with respect to the $\left.H^{[\gamma]}\right]_{\text {-norm. }}$.

Now we consider the infinitesimal deformation operator $\mathcal{V}_{M}$ for the constant Hermitian scalar curvature equation on $\left(M: \omega_{M}\right)$. Note that

$$
\begin{aligned}
\mathcal{V}_{M} \bullet \equiv & \frac{\omega_{M}^{(-1+m)}}{(-1+m) !} \wedge i(\bar{\partial} \partial)_{M} \frac{\Delta_{M} \bullet}{4 \pi}+m \cdot \frac{e\left(K_{M}\right)}{\omega_{M}} \cdot \frac{\omega_{M}^{(-1+m)}}{(-1+m) !} \wedge i(\bar{\partial} \partial)_{M} \bullet \\
& +\frac{(-1+m) \cdot \omega_{M}^{(-2+m)}}{(-1+m) !} \wedge i(\bar{\partial} \partial)_{M} \bullet \wedge \check{\pi}^{*} \operatorname{trace}\left(\frac{i}{2 \pi} F_{H_{M}}\right) .
\end{aligned}
$$

Standard theory of elliptic linear partial differential equations shows that

$$
\|f\|_{H^{[4]}} \leq C \cdot\left\|\mathcal{V}_{M} f\right\|
$$

$\forall f \in \Gamma(M: \mathbb{R})$ satisfying the normalization condition

$$
\int_{M} f \cdot \Omega_{M}=0
$$

Here $C>0$ is one constant independent of $N$ and $k$. 


\section{Linearizations of the constant Hermitian scalar curvature equation at approximate solutions}

Our goal in this section is to establish (II.12) and Proposition II.A. These results will be used in the next section to establish apriori estimates for $\psi \in \Gamma(\mathbb{P}(E): \mathbb{R})$ satisfying the normalization condition $\int_{\mathbb{P}(E)} \psi \cdot \Omega=0$. Most efforts in this section are devoted to the simplification of the expression of the linearizations of the CHSC equation at approximate solutions. Before proceeding we introduce some guidelines for the simplification process. Two linear partial differential operators

$$
P_{[k]} \equiv\left[\Delta_{V} \circ\left(-4 \pi \cdot n \cdot i d .+\Delta_{V}\right)+\Delta_{V} \circ \frac{\Delta_{M}}{k}+\frac{\Delta_{M}}{k} \circ \Delta_{V}+\frac{\Delta_{M}}{k} \circ \frac{\Delta_{M}}{k}\right]
$$

and

$$
\frac{1}{k \cdot k} \cdot \frac{\mathcal{V}_{M}}{\Omega_{M}}
$$

are of central importance. Note that $P_{[k]}$, when acting on the different components of $\Gamma(\mathbb{P}(E): \mathbb{R})=\Gamma(M: \mathbb{R}) \oplus \Gamma(M: W) \oplus \tilde{\sigma} \circ \Gamma(\mathbb{P}(E): \mathbb{R})$, has different degeneracy speeds because $P_{[k]}$ depends on the parameter $k \in \mathbb{N}$. When $P_{[k]}$ acts on $\tilde{\sigma} \circ \Gamma(\mathbb{P}(E): \mathbb{R})$ we have at least $L^{2}$ estimate, independent of $k \in \mathbb{N}$, as will be indicated in (III.2). But when $P_{[k]}$ acts on $\Gamma(M: W)$ the estimate we have, as will be indicated in (III.1), does have degeneracy speed of magnitude $O\left(\frac{1}{k}\right)$. So when dealing with the linearizations of the CHSC equation at approximate solutions any partial differential operator, with degeneracy speed of magnitude $\leq O\left(\frac{k^{m}}{k}\right)$, must be considered carefully when acting on $\Gamma(M: W)$. Similarly when dealing with the linearizations of the CHSC equation at approximate solutions any partial differential operator, with degeneracy speed of magnitude $\leq O\left(\frac{k^{m}}{k \cdot k}\right)$, must be considered carefully when acting on $\Gamma(M: \mathbb{R})$.It turns out that when computing the linearizations of the CHSC equation at approximate solutions two different leading linear partial differential operators are found:

- When acting on $\Gamma(M: W) \oplus \tilde{\sigma} \circ \Gamma(\mathbb{P}(E): \mathbb{R})$ the leading linear partial differential operator is $k^{m} \cdot \frac{(-2+m+n) !}{8 \pi} \cdot P_{[k]}$.

- When acting on $\Gamma(M: \mathbb{R})$ the leading linear partial differential operator is $\frac{k^{m}}{k \cdot k} \cdot(-2+m+n) ! \cdot \frac{\mathcal{V}_{M}}{\Omega_{M}}$.

It is exactly this phenomenon which makes the derivation of the linearizations of the CHSC equation at approximate solutions rather tedious: When considering the linearizations of the CHSC equation, depending on $k \in \mathbb{N}$, acting on different components of $\Gamma(\mathbb{P}(E): \mathbb{R})$ 
attention to be paid to the various partial differential operators involved is inhomogeneous - it is done according to the magnitudes of degeneracy they carry.

-Some notation

It takes lengthy calculation to express the constant Hermitian scalar curvature equation effectively. Here we want to introduce some notation to simplify the expression of the linearizations of the CHSC equation at approximate solutions. Suppose that $U$ is one coordinate open subset of $\mathbb{P}(E)$. Let $\Gamma(U: \mathbb{R})$ denote the space of smooth functions on $U$. Then we can form the ring $\Gamma(U: \mathbb{R})\left[\frac{1}{k}\right]$ consisting of polynomials in the parameter $\frac{1}{k}$ with coefficients in $\Gamma(U: \mathbb{R})$. Note that the sub-class of elements in $\Gamma(U: \mathbb{R})\left[\frac{1}{k}\right]$ with the zero-th order terms being nowhere zero on $U$ forms one multiplicative monoid. We will denote this multiplicative monoid by ${ }_{U} \mathrm{M}\left[\frac{1}{k}\right]$.

Now we elaborate on the genuine meaning of the symbol

$$
O_{Z}\left(k^{-\theta}\right)
$$

introduced in [9]. Here $Z$ is one vector bundle over $\mathbb{P}(E)$, and $\theta \in \mathbb{Z}$ satisfies $\theta \geq 0$. We start by treating the case $\theta=0 . O_{Z}\left(k^{-0}\right)$ is used to indicate one family of smooth sections of $Z$ over $\mathbb{P}(E)$, depending on the parameter $\frac{1}{k}$, such that over each coordinate open subset $U$ of $\mathbb{P}(E), O_{Z}\left(k^{-0}\right)$ can be expressed as the quotient of one polynomial in $\frac{1}{k}$, with "coefficients" in $\Gamma(U: Z)$, divided by some element in ${ }_{U} \mathrm{M}\left[\frac{1}{k}\right]$. In our context, $Z$ is usually related to some natural bundle over $\mathbb{P}(E)$ whose transition functions are certainly independent of the parameter $\frac{1}{k}$. Thus it is clear that $O_{Z}\left(k^{-0}\right)$ admits formal power series expansion in $\frac{1}{k}$ with "coefficients" being globally defined smooth sections of $Z$ over $\mathbb{P}(E)$. Now, for $\theta>0, O_{Z}\left(k^{-\theta}\right)$ simply means $k^{-\theta} \cdot O_{Z}\left(k^{-0}\right)$. We also adopt the convention, as in [9], that when $Z$ is the trivial bundle $\mathbb{R} \times \mathbb{P}(E)$ over $\mathbb{P}(E)$ the symbol $O\left(k^{-\theta}\right)$ will be used instead.

We demonstrate the ideas by considering the case where $Z$ is the trivial bundle $Z=\mathbb{R} \times \mathbb{P}(E)$ over $\mathbb{P}(E)$ related to the holomorphic tangent bundle of $\mathbb{P}(E)$. Assume that ${ }_{o} H_{[k]}$ and ${ }_{N} H_{[k]}$ are respectively the Kähler metrics on $\mathbb{P}(E)$ induced by the Kähler forms ${ }_{o} \omega_{[k]}$ and ${ }_{N} \omega_{[k]}$. Then $\operatorname{det}{ }_{o} H_{[k]}$ and $\operatorname{det}{ }_{N} H_{[k]}$ are essentially not functions on $\mathbb{P}(E)$. But

$$
\frac{\operatorname{det}{ }_{N} H_{[k]}}{\operatorname{det}{ }_{o} H_{[k]}}
$$


is. Thus $\frac{\operatorname{det}_{N} H_{[k]}}{\operatorname{det}_{o} H_{[k]}}$ is one element of the form $O_{Z}\left(k^{-0}\right)$. However it is usually impossible to express $\frac{\operatorname{det}_{N} H_{[k]}}{\operatorname{det}{ }_{o} H_{[k]}}$ as the quotient of globally defined functions on $\mathbb{P}(E)$. This example is central to our consideration: all the other cases considered in our context are similar to this situation.

Similar consideration applies to partial differential operators on $\mathbb{P}(E)$. Suppose that $U$ is one coordinate open subset of $\mathbb{P}(E)$. We will use the symbol $U_{U} \mathfrak{D}^{\emptyset}$ to indicate the class of linear partial differential operators on $U$, acting on functions, with the zero-th order terms being identically zero. Thus for $D \in U \mathfrak{D}^{\emptyset}$ we have

$$
D(f)=D(f+\text { constant })
$$

for any smooth function $f \in \Gamma(U: \mathbb{R})$ on $U$. The symbol $\stackrel{d}{U}^{\emptyset}$ is then used to indicate the sub-class of linear partial differential operators in ${ }_{U} \mathfrak{D}^{\emptyset}$ with order $\leq d .{ }_{U}^{d} \mathfrak{D}^{\emptyset}\left[\frac{1}{k}\right]$ is thus the ring of polynomials in $\frac{1}{k}$ with "coefficients" in ${ }_{U}^{d} \mathfrak{D}^{\emptyset}$. We will use the symbol $d D^{\emptyset}\left(k^{-0}\right)$ to indicate one family of linear partial differential operators acting on functions on $\mathbb{P}(E)$, depending on the parameter $\frac{1}{k}$, such that, on each coordinate open subset $U$ of $\mathbb{P}(E),{ }^{d} D^{\emptyset}\left(k^{-0}\right)$ can be expressed as the quotient of one element in ${ }_{U}^{d} \mathfrak{D}^{\emptyset}\left[\frac{1}{k}\right]$ divided by some element in ${ }_{U} \mathrm{M}\left[\frac{1}{k}\right]$. Besides it is always assumed that ${ }^{d} D^{\emptyset}\left(k^{-0}\right)$ admits formal power series expansion in $\frac{1}{k}$ with "coefficients" being globally defined linear partial differential operators on $\mathbb{P}(E)$. Actually, in our context, ${ }^{d} D^{\emptyset}\left(k^{-0}\right)$ always appears as some natural partial differential operators acting on functions related to certain natural bundles over $\mathbb{P}(E)$ whose transition functions are certainly independent of the parameter $\frac{1}{k}$. Now, for $\theta>0$, the symbol ${ }^{d} D^{\emptyset}\left(k^{-\theta}\right)$ will be used to indicate one element of the form $k^{-\theta} \cdot{ }^{d} D^{\emptyset}\left(k^{-0}\right)$.

Note that the class of linear derivation operators along the fiber directions of the fiber bundle $\check{\pi}: \mathbb{P}(E) \longrightarrow M$ is closed under the Lie bracket operation. We will use, for each $\theta \geq 0$, the symbol ${ }^{d} D_{V}^{\emptyset}\left(k^{-\theta}\right)$ to indicate one linear partial differential operator of the form ${ }^{d} D^{\emptyset}\left(k^{-\theta}\right)$ involving only derivation operators along the fiber directions of $\check{\pi}: \mathbb{P}(E) \longrightarrow M$.

Here we indicate a simple fact which will be used frequently but without being mentioned explicitly in the rest of this section: Given a function $f \in \Gamma(M: W)$ the $L^{2}$ (or $L^{\infty}$ ) norm of ${ }^{d} D_{V}^{\emptyset}\left(k^{-0}\right) f$ on each fiber of $\check{\pi}: \mathbb{P}(E) \longrightarrow M$ is controlled by the $L^{2}$ (or $L^{\infty}$ ) norm of $f$ on the same fiber in a uniform manner over $M$. Because of this we sometimes 
write (less precisely) ${ }^{2} D^{\emptyset}\left(k^{-0}\right) \circ \sigma$ for ${ }^{2} D^{\emptyset}\left(k^{-0}\right) \circ{ }^{d} D_{V}^{\emptyset}\left(k^{-0}\right) \circ \sigma$.

-Explicit expression of the linearizations of the CHSC equation at approximate solutions

Assume that $\psi$ is a smooth $\mathbb{R}$-valued function on $\mathbb{P}(E)$ satisfying the normalization condition

$$
\int_{\mathbb{P}(E)} \psi \cdot \Omega=0
$$

Let

$$
{ }_{N} \omega_{[k]}<t \cdot \psi>\equiv{ }_{N} \omega_{[k]}+t \cdot i \bar{\partial} \partial \psi
$$

and ${ }_{N} H_{[k]}<t \cdot \psi>$ be the Kähler metric on $\mathbb{P}(E)$ induced by ${ }_{N} \omega_{[k]}<t \cdot \psi>$. Then we have

$$
\begin{array}{r}
\left.\frac{d}{d t}\left(\left({ }_{N} \omega_{[k]}<t \cdot \psi\right\rangle\right)^{(-2+m+n)} \wedge\left(-c_{k} \cdot{ }_{N} \omega_{[k]}<t \cdot \psi>+\frac{i}{2 \pi} \bar{\partial} \partial \log \operatorname{det}{ }_{N} H_{[k]}<t \cdot \psi>\right)\right)\left.\right|_{t=0} \\
={ }_{N} \mathfrak{S}_{[k]}(\psi)
\end{array}
$$

in which

$$
\begin{aligned}
{ }_{N} \mathfrak{S}_{[k]}(\psi) \equiv & (-2+m+n) \cdot{ }_{N} \omega_{[k]}^{(-3+m+n)} \wedge i \bar{\partial} \partial \psi \wedge \frac{i}{2 \pi} \bar{\partial} \partial \log \operatorname{det}{ }_{N} H_{[k]} \\
& +{ }_{N} \omega_{[k]}^{(-2+m+n)} \wedge \frac{i}{2 \pi} \bar{\partial} \partial\left(\frac{\left.\frac{d}{d t}\left(\operatorname{det}{ }_{N} H_{[k]}<t \cdot \psi>\right)\right|_{t=0}}{\operatorname{det}{ }_{N} H_{[k]}}\right) \\
& -c_{k} \cdot(-1+m+n) \cdot{ }_{N} \omega_{[k]}^{(-2+m+n)} \wedge i \bar{\partial} \partial \psi
\end{aligned}
$$

Note that ${ }_{N} \mathfrak{S}_{[k]}$ is the linearization of the CHSC Equation at the approximate solution ${ }_{N} \omega_{[k]}$.

In the following computation the representative $\left(\frac{i}{2 \pi} F_{H_{L^{*}}}\right)$ of $e\left(L^{*}\right)$ on $\mathbb{P}(E)$ will be fixed. Thus $e\left(L^{*}\right)$ will be identified with $\left(\frac{i}{2 \pi} F_{H_{L^{*}}}\right)$ when there is no confusion.

We will now compute those terms on the right-hand side of (II.1) more explicitly. Note that

$$
\log \operatorname{det}{ }_{N} H_{[k]}=\log \operatorname{det}{ }_{o} H_{[k]}+\frac{1}{k} \cdot\left(\frac{\Delta_{V} \phi_{1}}{2}+\frac{\Delta_{M} \phi_{0}}{2}\right)+O\left(k^{-2}\right)
$$


and

$$
\begin{aligned}
& \frac{\left.\frac{d}{d t}\left(\operatorname{det}_{N} H_{[k]}<t \cdot \psi>\right)\right|_{t=0}}{\operatorname{det}{ }_{N} H_{[k]}} \\
& =\left(\frac{\Delta_{V} \psi}{2}+\frac{1}{k} \cdot Q_{V}\left(\psi: \phi_{1}\right)\right) \cdot\left(1+\frac{1}{k} \cdot \frac{\Delta_{V} \phi_{1}}{2}+O\left(k^{-2}\right)\right)^{-1} \\
& \quad+\frac{1}{k} \cdot \frac{\Delta_{M} \psi}{2}+\frac{1}{k \cdot k} \cdot\left(\frac{Q_{m}\left(\psi: \phi_{1}\right)}{2 \pi}+Q_{M}(\psi)\right) \\
& \quad+\frac{1}{k \cdot k} \cdot \frac{-\Delta_{M} \psi}{2} \cdot\left(-m \cdot \frac{e(E)}{\omega_{M}}+\frac{\Delta_{M} \phi_{0}}{2}\right) \\
& \quad+{ }^{2} D_{V}^{\emptyset}\left(k^{-2}\right) \psi+{ }^{2} D^{\emptyset}\left(k^{-3}\right) \psi .
\end{aligned}
$$

Let $\mathfrak{c}_{k} \equiv(-1+m+n) \cdot c_{k}$. Then we have

$$
\begin{aligned}
& -\mathfrak{c}_{k} \cdot{ }_{N} \omega_{[k]}^{(-2+m+n)} \\
& =-n(-1+n) \cdot \frac{(-2+m+n) !}{m ! \cdot(-2+n) !} \cdot e\left(L^{*}\right)^{(-2+n)} \wedge\left(k \cdot \omega_{M}\right)^{m} \\
& -n(-1+n) \cdot \frac{(-2+m+n) !}{(-1+m) ! \cdot(-1+n) !} \\
& \cdot e\left(L^{*}\right)^{(-1+n)} \wedge\left(k \cdot \omega_{M}\right)^{(-1+m)} \\
& -n(-1+n) \cdot \frac{(-2+m+n) !}{(m-1) ! \cdot(-1+n) !} \cdot(-1+n) \\
& \cdot e\left(L^{*}\right)^{(-2+n)} \wedge \frac{i \bar{\partial} \partial \phi_{1}}{k} \wedge\left(k \cdot \omega_{M}\right)^{(-1+m)} \\
& +\frac{1}{k} \cdot m \cdot \frac{e\left(K_{M}\right)}{\omega_{M}} \cdot \frac{(-2+m+n) !}{(-1+m) ! \cdot(-1+n) !} \\
& \cdot e\left(L^{*}\right)^{(-1+n)} \wedge\left(k \cdot \omega_{M}\right)^{(-1+m)} \\
& -n(-1+n) \cdot \frac{(-2+m+n) !}{(-2+m) ! \cdot n !} \\
& \cdot\left(e\left(L^{*}\right)+i \bar{\partial} \partial \phi_{0}\right)^{n} \wedge\left(k \cdot \omega_{M}\right)^{(-2+m)} \\
& -n(-1+n) \cdot \frac{(-2+m+n) !}{m ! \cdot(-2+n) !} \cdot(-2+n) \\
& \cdot e\left(L^{*}\right)^{(-3+n)} \wedge \frac{i \bar{\partial} \partial \phi_{1}}{k} \wedge\left(k \cdot \omega_{M}\right)^{m}
\end{aligned}
$$




$$
\begin{aligned}
& +\frac{1}{k} \cdot m \cdot \frac{e\left(K_{M}\right)}{\omega_{M}} \cdot \frac{(-2+m+n) !}{m ! \cdot(-2+n) !} \\
& \cdot e\left(L^{*}\right)^{(-2+n)} \wedge\left(k \cdot \omega_{M}\right)^{m} \\
& +k^{m} \cdot O_{\wedge(-2+n)} T^{*}(\mathbb{P}(E)) \\
& +k^{m} \cdot O_{\wedge^{(-2+m+n)} T^{*}(\mathbb{P}(E))}\left(k^{-3}\right) .
\end{aligned}
$$

By using the equalities (II.2)-(II.4) we can now express (II.1) more explicitly. We introduce one linear partial differential operator

$$
\mathfrak{L}_{[k]}: \Gamma(\mathbb{P}(E): \mathbb{R}) \longrightarrow \Gamma\left(\mathbb{P}(E): \wedge^{\max } T^{*}(\mathbb{P}(E))\right)
$$

on $\mathbb{P}(E)$ as follows:

$$
\begin{aligned}
& \mathfrak{L}_{[k]} \psi=\frac{(-2+m+n) !}{m ! \cdot(-3+n) !} \cdot e\left(L^{*}\right)^{(-3+n)} \wedge\left(k \cdot \omega_{M}\right)^{m} \wedge i(\bar{\partial} \partial)_{V} \psi \wedge \frac{i}{2 \pi}(\bar{\partial} \partial)_{V} \log \operatorname{det}{ }_{o} H_{[k]} \\
& +\frac{(-2+m+n) !}{(-2+n) ! \cdot(-1+m) !} \cdot e\left(L^{*}\right)^{(-2+n)} \wedge\left(k \cdot \omega_{M}\right)^{(-1+m)} \wedge i(\bar{\partial} \partial)_{M} \psi \wedge \frac{i}{2 \pi}(\bar{\partial} \partial)_{V} \log \operatorname{det}{ }_{o} H_{[k]} \\
& +\frac{(-2+m+n) !}{(-1+n) ! \cdot(-2+m) !} \cdot e\left(L^{*}\right)^{(-1+n)} \wedge\left(k \cdot \omega_{M}\right)^{(-2+m)} \wedge i(\bar{\partial} \partial)_{M} \psi \wedge \frac{i}{2 \pi} \bar{\partial} \partial \log \operatorname{det}{ }_{o} H_{[k]} \\
& +\frac{(-2+m+n) !}{(-2+n) ! \cdot m !} \cdot e\left(L^{*}\right)^{(-2+n)} \wedge\left(k \cdot \omega_{M}\right)^{m} \wedge \frac{i}{2 \pi}(\bar{\partial} \partial)_{V}\left(\frac{\Delta_{V} \psi}{2}\right) \\
& +\frac{(-2+m+n) !}{(-2+n) ! \cdot m !} \cdot e\left(L^{*}\right)^{(-2+n)} \wedge\left(k \cdot \omega_{M}\right)^{m} \wedge \frac{i}{2 \pi}(\bar{\partial} \partial)_{V}\left(\frac{1}{k} \cdot \frac{\Delta_{M} \psi}{2}\right) \\
& +\frac{(-2+m+n) !}{(-1+n) ! \cdot(-1+m) !} \cdot e\left(L^{*}\right)^{(-1+n)} \wedge\left(k \cdot \omega_{M}\right)^{(-1+m)} \wedge \frac{i}{2 \pi}(\bar{\partial} \partial)_{M}\left(\frac{\Delta_{V} \psi}{2}\right) \\
& +\frac{(-2+m+n) !}{(-1+n) ! \cdot(-1+m) !} \cdot e\left(L^{*}\right)^{(-1+n)} \wedge\left(k \cdot \omega_{M}\right)^{(-1+m)} \wedge \frac{i}{2 \pi}(\bar{\partial} \partial)_{M}\left(\frac{1}{k} \cdot \frac{\Delta_{M} \psi}{2}\right) \\
& +\frac{(-2+m+n) !}{(-2+n) ! \cdot m !} \cdot e\left(L^{*}\right)^{(-2+n)} \wedge\left(k \cdot \omega_{M}\right)^{m} \wedge \frac{1}{k} \cdot \frac{i}{2 \pi}(\bar{\partial} \partial)_{V}\left(-\frac{\Delta_{V} \phi_{1}}{2} \cdot \frac{\Delta_{V} \psi}{2}+Q_{V}\left(\psi: \phi_{1}\right)\right) \\
& -n(-1+n) \cdot \frac{(-2+m+n) !}{(-2+n) ! \cdot m !} \cdot e\left(L^{*}\right)^{(-2+n)} \wedge\left(k \cdot \omega_{M}\right)^{m} \wedge i(\bar{\partial} \partial)_{V} \psi \\
& -n(-1+n) \cdot \frac{(-2+m+n) !}{(-1+n) ! \cdot(-1+m) !} \cdot e\left(L^{*}\right)^{(-1+n)} \wedge\left(k \cdot \omega_{M}\right)^{(-1+m)} \wedge i(\bar{\partial} \partial)_{M} \psi \\
& +\frac{1}{k} \cdot m \cdot \frac{e\left(K_{M}\right)}{\omega_{M}} \cdot \frac{(-2+m+n) !}{(-1+n) ! \cdot(-1+m) !} \cdot e\left(L^{*}\right)^{(-1+n)} \wedge\left(k \cdot \omega_{M}\right)^{(-1+m)} \wedge i(\bar{\partial} \partial)_{M} \psi \\
& -n(-1+n) \cdot \frac{(-2+m+n) !}{n ! \cdot(-2+m) !} \cdot e\left(L^{*}\right)^{n} \wedge\left(k \cdot \omega_{M}\right)^{(-2+m)} \wedge i(\bar{\partial} \partial)_{M} \psi \cdot
\end{aligned}
$$

Then we find that

$$
\begin{aligned}
{ }_{N} \mathfrak{S}_{[k]}(\psi)= & \mathfrak{L}_{[k]} \psi+\mathfrak{B}_{[k]} \psi \\
& +\mathfrak{C}_{[k]} \psi+k^{m} \cdot e\left(L^{*}\right)^{(-1+n)} \wedge \omega_{M}^{m} \cdot{ }^{4} D_{V}^{\emptyset}\left(k^{-2}\right) \psi \\
& +k^{m} \cdot e\left(L^{*}\right)^{(-1+n)} \wedge \omega_{M}^{m} \cdot{ }^{4} D^{\emptyset}\left(k^{-3}\right) \psi,
\end{aligned}
$$

in which the linear partial differential operators $\mathfrak{B}_{[k]}$ and $\mathfrak{C}_{[k]}$, bringing $\Gamma(\mathbb{P}(E): \mathbb{R})$ to $\Gamma\left(\mathbb{P}(E): \wedge^{\max } T^{*}(\mathbb{P}(E))\right)$, are respectively defined 
as follows:

$$
\begin{aligned}
& \mathfrak{B}_{[k]} \psi=\frac{(-2+m+n) !}{m ! \cdot(-3+n) !} \cdot(-3+n) \cdot e\left(L^{*}\right)^{(-4+n)} \wedge \frac{i(\bar{\partial} \partial)_{V} \phi_{1}}{k} \wedge\left(k \cdot \omega_{M}\right)^{m} \wedge \\
& i(\bar{\partial} \partial)_{V} \psi \wedge \frac{i}{2 \pi}(\bar{\partial} \partial)_{V} \log \operatorname{det}{ }_{o} H_{[k]} \\
& +\frac{(-2+m+n) !}{m ! \cdot(-3+n) !} \cdot e\left(L^{*}\right)^{(-3+n)} \wedge\left(k \cdot \omega_{M}\right)^{m} \wedge i(\bar{\partial} \partial)_{V} \psi \wedge \frac{1}{k} \cdot \frac{i}{2 \pi}(\bar{\partial} \partial)_{V}\left(\frac{\Delta_{V} \phi_{1}}{2}+\frac{\Delta_{M} \phi_{0}}{2}\right) \\
& +\frac{(-2+m+n) !}{(-1+m) ! \cdot(-2+n) !} \cdot e\left(L^{*}\right)^{(-2+n)} \wedge\left(k \cdot \omega_{M}\right)^{(-1+m)} \wedge i(\bar{\partial} \partial)_{V} \psi \wedge \frac{i}{2 \pi} \bar{\partial} \partial \log \operatorname{det}{ }_{o} H_{[k]} \\
& +\frac{(-2+m+n) !}{(-1+m) ! \cdot(-2+n) !} \cdot(-2+n) \cdot e\left(L^{*}\right)^{(-3+n)} \wedge i(\bar{\partial} \partial)_{M} \phi_{0} \wedge \\
& \left(k \cdot \omega_{M}\right)^{(-1+m)} \wedge i(\bar{\partial} \partial)_{V} \psi \wedge \frac{i}{2 \pi}(\bar{\partial} \partial)_{V} \log \operatorname{det}{ }_{o} H_{[k]} \\
& +\frac{(-2+m+n) !}{m ! \cdot(-2+n) !} \cdot(-2+n) \cdot e\left(L^{*}\right)^{(-3+n)} \wedge \frac{i(\bar{\partial} \partial)_{V} \phi_{1}}{k} \wedge\left(k \cdot \omega_{M}\right)^{m} \wedge \frac{i}{2 \pi}(\bar{\partial} \partial)_{V}\left(\frac{\Delta_{V} \psi}{2}\right) \\
& +\frac{(-2+m+n) !}{(-1+m) ! \cdot(-1+n) !} \cdot e\left(L^{*}\right)^{(-1+n)} \wedge\left(k \cdot \omega_{M}\right)^{(-1+m)} \wedge \frac{i}{2 \pi}(\bar{\partial} \partial)_{V}\left(\frac{\Delta_{V} \psi}{2}\right) \\
& +\frac{(-2+m+n) !}{(-1+m) ! \cdot(-1+n) !} \cdot(-1+n) \cdot e\left(L^{*}\right)^{(-2+n)} \wedge i(\bar{\partial} \partial)_{M} \phi_{0} \wedge\left(k \cdot \omega_{M}\right)^{(-1+m)} \wedge \\
& \frac{i}{2 \pi}(\bar{\partial} \partial)_{V}\left(\frac{\Delta_{V} \psi}{2}\right) \\
& -n(-1+n) \cdot \frac{(-2+m+n) !}{m ! \cdot(-2+n) !} \cdot(-2+n) \cdot e\left(L^{*}\right)^{(-3+n)} \wedge \frac{i(\bar{\partial} \partial)_{V} \phi_{1}}{k} \wedge\left(k \cdot \omega_{M}\right)^{m} \wedge i(\bar{\partial} \partial)_{V} \psi \\
& +\frac{1}{k} \cdot m \cdot \frac{e\left(K_{M}\right)}{\omega_{M}} \cdot \frac{(-2+m+n) !}{m ! \cdot(-2+n) !} \cdot e\left(L^{*}\right)^{(-2+n)} \wedge\left(k \cdot \omega_{M}\right)^{m} \wedge i(\bar{\partial} \partial)_{V} \psi \\
& -n(-1+n) \cdot \frac{(-2+m+n) !}{(-1+m) ! \cdot(-1+n) !} \cdot e\left(L^{*}\right)^{(-1+n)} \wedge\left(k \cdot \omega_{M}\right)^{(-1+m)} \wedge i(\bar{\partial} \partial)_{V} \psi \\
& -n(-1+n) \cdot \frac{(-2+m+n) !}{(-1+m) ! \cdot(-1+n) !} \cdot(-1+n) \cdot e\left(L^{*}\right)^{(-2+n)} \wedge \\
& i(\bar{\partial} \partial)_{M} \phi_{0} \wedge\left(k \cdot \omega_{M}\right)^{(-1+m)} \wedge i(\bar{\partial} \partial)_{V} \psi
\end{aligned}
$$

and

$$
\begin{aligned}
\mathfrak{C}_{[k]} \psi= & \frac{(-2+m+n) !}{m ! \cdot(-2+n) !} \cdot e\left(L^{*}\right)^{(-2+n)} \wedge\left(k \cdot \omega_{M}\right)^{m} \wedge \frac{1}{k \cdot k} \cdot \frac{i}{2 \pi}(\bar{\partial} \partial)_{V}\left(\frac{Q m\left(\psi: \phi_{1}\right)}{2 \pi}+Q_{M}(\psi)\right) \\
& +\frac{(-2+m+n) !}{m ! \cdot(-2+n) !} \cdot e\left(L^{*}\right)^{(-2+n)} \wedge\left(k \cdot \omega_{M}\right)^{m} \wedge \\
& \frac{1}{k \cdot k} \cdot \frac{i}{2 \pi}(\bar{\partial} \partial)_{V}\left[\frac{-\Delta_{M} \psi}{2} \cdot\left(-m \cdot \frac{e(E)}{\omega_{M}}+\frac{\Delta_{M} \phi_{0}}{2}\right)\right] \\
& +\frac{(-2+m+n) !}{m ! \cdot(-2+n) !} \cdot(-2+n) \cdot e\left(L^{*}\right)^{(-3+n)} \wedge \frac{i(\bar{\partial} \partial)_{V} \phi_{1}}{k} \wedge\left(k \cdot \omega_{M}\right)^{m} \wedge \frac{i}{2 \pi}(\bar{\partial} \partial)_{V}\left(\frac{1}{k} \cdot \frac{\Delta_{M} \psi}{2}\right) \\
& +\frac{(-2+m+n) !}{(-1+m) ! \cdot(-1+n) !} \cdot e\left(L^{*}\right)^{(-1+n)} \wedge\left(k \cdot \omega_{M}\right)^{(-1+m)} \wedge \frac{i}{2 \pi}(\bar{\partial} \partial)_{V}\left(\frac{1}{k} \cdot \frac{\Delta_{M} \psi}{2}\right) \\
& +\frac{(-2+m+n) !}{(-1+m) ! \cdot(-1+n) !} \cdot(-1+n) \cdot e\left(L^{*}\right)^{(-2+n)} \wedge\left(k \cdot \omega_{M}\right)^{(-1+m)} \wedge \\
& +\frac{(-2+m+n) !}{(-1+m) ! \cdot(-1+n) !} \cdot(-1+n) \cdot e\left(L^{*}\right)^{(-2+n)} \wedge\left(k \cdot \omega_{M}\right)^{(-1+m)} \wedge \\
& \frac{i(\bar{\partial} \partial)_{m} \phi_{1}}{k} \wedge \frac{i}{2 \pi}(\bar{\partial} \partial)_{m}\left(\frac{\Delta_{V} \psi}{2}\right) \\
& +\frac{(-2+m+n) !}{(-1+m) ! \cdot(-1+n) !} \cdot e\left(L^{*}\right)^{(-1+n)} \wedge\left(k \cdot \omega_{M}\right)^{(-1+m)}\left(\frac{1}{k} \cdot \frac{\Delta_{M} \psi}{2}\right) \\
\text { (II.8) } & \frac{1}{k} \cdot \frac{i}{2 \pi}(\bar{\partial} \partial)_{M}\left(-\frac{\Delta_{V} \phi_{1}}{2} \cdot \frac{\Delta_{V} \psi}{2}+Q_{V}\left(\psi: \phi_{1}\right)\right)
\end{aligned}
$$




$$
\begin{aligned}
& +\frac{(-2+m+n) !}{(-1+m) ! \cdot(-1+n) !} \cdot(-1+n) \cdot e\left(L^{*}\right)^{(-2+n)} \wedge\left(k \cdot \omega_{M}\right)^{(-1+m)} \wedge \frac{i(\bar{\partial} \partial)_{V} \phi_{1}}{k} \wedge \frac{i(\bar{\partial} \partial)_{M}}{2 \pi}\left(\frac{\Delta_{V} \psi}{2}\right) \\
& +\frac{(-2+m+n) !}{(-2+m) ! \cdot n !} \cdot e\left(L^{*}\right)^{n} \wedge\left(k \cdot \omega_{M}\right)^{(-2+m)} \wedge \frac{i}{2 \pi}(\bar{\partial} \partial)_{M}\left(\frac{\Delta_{V} \psi}{2}\right) \\
& +\frac{(-2+m+n) !}{(-2+m) ! \cdot n !} \cdot n \cdot e\left(L^{*}\right)^{(-1+n)} \wedge i(\bar{\partial} \partial)_{M} \phi_{0} \wedge\left(k \cdot \omega_{M}\right)^{(-2+m)} \wedge \frac{i}{2 \pi}(\bar{\partial} \partial)_{M}\left(\frac{\Delta_{V} \psi}{2}\right) .
\end{aligned}
$$

Since the detailed terms of those items on the right-hand side of (II.6) are still rather complicated, we need to simplify (II. 6) further. So we compute the term $\frac{i}{2 \pi} \bar{\partial} \partial \log \operatorname{det}{ }_{o} H_{[k]}$ involved in (II.5) and (II.7). Note that

$$
\begin{aligned}
\frac{i}{2 \pi} \bar{\partial} \partial \log \operatorname{det}{ }_{o} H_{[k]}= & \cdot\left(\frac{i}{2 \pi} F_{H_{L^{*}}}\right) \\
& +\check{\pi}^{*} \operatorname{trace}\left(\frac{i}{2 \pi} F_{H_{E}}\right)+\check{\pi}^{*} \operatorname{trace}\left(\frac{i}{2 \pi} F_{H_{M}}\right) \\
& +O_{\mathcal{C}_{M}}\left(k^{-1}\right)+O_{\mathcal{C}_{m}}\left(k^{-2}\right)+O_{\mathcal{C}_{V}}\left(k^{-2}\right)
\end{aligned}
$$

and $\frac{i}{2 \pi}(\bar{\partial} \partial)_{V} \log \operatorname{det}{ }_{o} H_{[k]}$ induces the Fubini-Study Kähler form on each fiber of $\check{\pi}: \mathbb{P}(E) \longrightarrow M$. (It is easy to establish (II.9) using the Special Coordinate System introduced in the Appendix.) Thus we have the following results:

$$
\mathfrak{B}_{[k]} \psi=\widehat{\mathfrak{B}}_{[k]} \psi+k^{m} \cdot e\left(L^{*}\right)^{(-1+n)} \wedge \omega_{M}^{m} \cdot{ }^{4} D_{V}^{\emptyset}\left(k^{-2}\right) \psi
$$

and

$$
\begin{aligned}
\mathfrak{L}_{[k]} \psi= & \widehat{\mathfrak{L}}_{[k]} \psi+k^{m} \cdot e\left(L^{*}\right)^{(-1+n)} \wedge \omega_{M}^{m} \cdot{ }^{4} D_{V}^{\emptyset}\left(k^{-2}\right) \psi \\
& +k^{m} \cdot e\left(L^{*}\right)^{(-1+n)} \wedge \omega_{M}^{m} \cdot{ }^{4} D^{\emptyset}\left(k^{-3}\right) \psi .
\end{aligned}
$$

Here the linear partial differential operators

$$
\widehat{\mathfrak{B}}_{[k]}: \Gamma(\mathbb{P}(E): \mathbb{R}) \longrightarrow \Gamma\left(\mathbb{P}(E): \wedge^{\max } T^{*}(\mathbb{P}(E))\right)
$$

and

$$
\widehat{\mathfrak{L}}_{[k]}: \Gamma(\mathbb{P}(E): \mathbb{R}) \longrightarrow \Gamma\left(\mathbb{P}(E): \wedge^{\max } T^{*}(\mathbb{P}(E))\right)
$$

on $\mathbb{P}(E)$ are respectively defined by

$$
\begin{aligned}
\widehat{\mathfrak{B}}_{[k]} \psi= & \frac{(-2+m+n) !}{(-1+m) ! \cdot(-1+n) !} \cdot e\left(L^{*}\right)^{(-1+n)} \wedge\left(k \cdot \omega_{M}\right)^{(-1+m)} \\
\wedge \frac{i}{2 \pi}(\bar{\partial} \partial)_{V}\left(\frac{\Delta_{V} \psi}{2}\right) & \\
+ & \frac{(-2+m+n) !}{(-1+m) ! \cdot(-2+n) !} \cdot e\left(L^{*}\right)^{(-2+n)} \wedge\left(k \cdot \omega_{M}\right)^{(-1+m)} \\
& \wedge i(\bar{\partial} \partial)_{V} \psi \wedge \check{\pi}^{*} \operatorname{trace}\left(\frac{i}{2 \pi} F_{H_{E}}\right) \\
+ & \frac{(-2+m+n) !}{m ! \cdot(-2+n) !} \cdot(-2+n) \cdot e\left(L^{*}\right)^{(-3+n)} \wedge \frac{i(\bar{\partial} \partial)_{V} \phi_{1}}{k} \\
& \wedge\left(k \cdot \omega_{M}\right)^{m} \wedge\left[-n \cdot i(\bar{\partial} \partial)_{V} \psi+\frac{i}{2 \pi}(\bar{\partial} \partial)_{V}\left(\frac{\Delta_{V} \psi}{2}\right)\right]
\end{aligned}
$$


and

$$
\begin{aligned}
\widehat{\mathfrak{L}}_{[k]} \psi= & k^{m} \cdot \frac{(-2+m+n) !}{m ! \cdot(-1+n) !} \cdot e\left(L^{*}\right)^{(-1+n)} \wedge \omega_{M}^{m} \\
& \cdot \frac{1}{8 \pi}\left[\Delta_{V} \circ\left(-4 \pi \cdot n \cdot \psi+\Delta_{V} \psi\right)\right. \\
& \left.\quad+\Delta_{V} \frac{\Delta_{M}}{k} \psi+\frac{\Delta_{M}}{k} \Delta_{V} \psi+\frac{\Delta_{M}}{k} \frac{\Delta_{M}}{k} \psi\right] \\
+ & \frac{k^{m}}{k \cdot k} \cdot m \cdot \frac{e\left(K_{M}\right)}{\omega_{M}} \cdot \frac{(-2+m+n) !}{(-1+n) ! \cdot(-1+m) !} \cdot e\left(L^{*}\right)^{(-1+n)} \wedge \\
& \omega_{M}^{(-1+m)} \wedge i(\bar{\partial} \partial)_{M} \psi \\
+ & \frac{k^{m}}{k \cdot k} \cdot \frac{(-2+m+n) !}{(-1+n) ! \cdot(-2+m) !} \cdot e\left(L^{*}\right)^{(-1+n)} \wedge \omega_{M}^{(-2+m)} \wedge \\
& \quad i(\bar{\partial} \partial)_{M} \psi \wedge \check{\pi}^{*} \operatorname{trace}\left(\frac{i}{2 \pi} F_{H_{M}}\right) \\
+ & \frac{k^{m}}{k} \cdot \frac{(-2+m+n) !}{(-1+n) ! \cdot m !} \cdot e\left(L^{*}\right)^{(-1+n)} \wedge \omega_{M}^{m} . \\
& \frac{\Delta_{V}}{4 \pi}\left(-\frac{\Delta_{V} \phi_{1}}{2} \cdot \frac{\Delta_{V} \psi}{2}+Q_{V}\left(\psi: \phi_{1}\right)\right) \\
+ & \frac{k^{m}}{k \cdot k} \cdot \frac{(-2+m+n) !}{(-1+n) ! \cdot(-2+m) !} \cdot \omega_{M}^{(-2+m)} \wedge i(\bar{\partial} \partial)_{M} \psi \wedge \\
& \quad\left(e\left(L^{*}\right)^{n}+e\left(L^{*}\right)^{(-1+n)} \wedge \check{\pi}^{*} \operatorname{trace}\left(\frac{i}{2 \pi} F_{H_{E}}\right)\right) .
\end{aligned}
$$

Hence we have established the following simpler expression of ${ }_{N} \mathfrak{S}_{[k]}$ :

$$
\begin{aligned}
&{ }_{N} \mathfrak{S}_{[k]} \psi=\widehat{\mathfrak{L}}_{[k]} \psi+\widehat{\mathfrak{B}}_{[k]} \psi+\mathfrak{C}_{[k]} \psi \\
& \\
&+k^{m} \cdot e\left(L^{*}\right)^{(-1+n)} \wedge \omega_{M}^{m} \cdot{ }^{4} D_{V}^{\emptyset}\left(k^{-2}\right) \psi \\
&+k^{m} \cdot e\left(L^{*}\right)^{(-1+n)} \wedge \omega_{M}^{m} \cdot{ }^{4} D^{\emptyset}\left(k^{-3}\right) \psi
\end{aligned}
$$

-Concise expression for $\widehat{\mathfrak{L}}_{[k]}+\widehat{\mathfrak{B}}_{[k]}+\mathfrak{C}_{[k]}$

Now we discuss some special properties of the expression (II.12) in order to understand ${ }_{N} \mathfrak{S}_{[k]}$ better. We start by simplifying the expression (II.8). We have, by applying the Einstein-Hermitian condition to 
the 4-th item on the right-hand side of (II.8),

$$
\begin{aligned}
\mathfrak{C}_{[k]} \psi= & \frac{k^{m}}{k \cdot k} \cdot \frac{(-2+m+n) !}{m ! \cdot(-1+n) !} \cdot e\left(L^{*}\right)^{(-1+n)} \wedge \omega_{M}^{m} \cdot \frac{\Delta_{V}}{4 \pi}\left(\frac{Q_{m}\left(\psi: \phi_{1}\right)}{2 \pi}+Q_{M}(\psi)\right) \\
& +\frac{k^{m}}{k \cdot k} \cdot \frac{(-2+m+n) !}{m ! \cdot(-1+n) !} \cdot e\left(L^{*}\right)^{(-1+n)} \wedge \omega_{M}^{m} \cdot \frac{\Delta_{V}}{4 \pi}\left[\frac{-\Delta_{M} \psi}{2} \cdot\left(-m \cdot \frac{e(E)}{\omega_{M}}+\frac{\Delta_{M} \phi_{0}}{2}\right)\right] \\
& +\frac{k^{m}}{k \cdot k} \cdot \frac{(-2+m+n) !}{m ! \cdot(-2+n) !} \cdot(-2+n) \cdot e\left(L^{*}\right)^{(-3+n)} \wedge i(\bar{\partial} \partial)_{V} \phi_{1} \wedge \omega_{M}^{m} \wedge \frac{i}{2 \pi}(\bar{\partial} \partial)_{V}\left(\frac{\Delta_{M} \psi}{2}\right) \\
& -\frac{k^{m}}{k \cdot k} \cdot \frac{\Lambda_{M}\left[\bar{\pi}^{*} \operatorname{trace}\left(\frac{i}{2 \pi} F_{H_{E}}\right)\right]}{n} \cdot \frac{(-2+m+n) !}{m ! \cdot(-1+n) !} \cdot e\left(L^{*}\right)^{(-1+n)} \wedge \omega_{M}^{m} \cdot \frac{\Delta_{V}}{8 \pi}\left(\Delta_{M} \psi\right) \\
& +\frac{k^{m}}{k \cdot k} \cdot \frac{(-2+m+n) !}{m ! \cdot(-1+n) !} \cdot e\left(L^{*}\right)^{(-1+n)} \wedge \omega_{M}^{m} \cdot \frac{\Delta_{M} \phi_{0}}{2} \cdot \frac{\Delta_{V}}{4 \pi}\left(\frac{\Delta_{M} \psi}{2}\right) \\
& +\frac{k^{m} m}{k \cdot k} \cdot \frac{(-2+m+n) !}{(-1+m) ! \cdot(-1+n) !} \cdot(-1+n) \\
& \cdot e\left(L^{*}\right)^{(-2+n)} \wedge i(\bar{\partial} \partial)_{m} \phi_{1} \wedge \omega_{M}^{(-1+m)} \wedge \frac{i}{2 \pi}(\bar{\partial} \partial)_{m}\left(\frac{\Delta_{V} \psi}{2}\right) \\
\text { II. } \underline{8}) \quad & +\frac{k^{m}}{k \cdot k} \cdot \frac{(-2+m+n) !}{m ! \cdot(-1+n) !} \cdot e\left(L^{*}\right)^{(-1+n)} \wedge \omega_{M}^{m} \cdot \frac{\Delta_{M}}{4 \pi}\left(-\frac{\Delta_{V} \phi_{1}}{2} \cdot \frac{\Delta_{V} \psi}{2}+Q_{V}\left(\psi: \phi_{1}\right)\right)+ \\
& \frac{k^{m}}{k \cdot k} \cdot \frac{(-2+m+n) !}{m ! \cdot(-1+n) !} \cdot e\left(L^{*}\right)^{(-1+n)} \wedge \omega_{M}^{m} \cdot \frac{\Delta_{V} \phi_{1}}{2} \cdot \frac{\Delta_{M}}{4 \pi}\left(\frac{\Delta_{V} \psi}{2}\right) \\
& +\frac{k^{m}}{k \cdot k} \cdot \frac{(-2+m+n) !}{(-2+m) ! \cdot n !} \cdot e\left(L^{*}\right)^{n} \wedge \omega_{M}^{(-2+m)} \wedge \frac{i}{2 \pi}(\bar{\partial} \partial)_{M}\left(\frac{\Delta_{V} \psi}{2}\right) \\
& +\frac{k^{m}}{k \cdot k} \cdot \frac{(-2+m+n) !}{(-2+m) ! \cdot n !} \cdot n \cdot e\left(L^{*}\right)^{(-1+n)} \wedge i(\bar{\partial} \partial)_{M} \phi_{0} \wedge \omega_{M}^{(-2+m)} \wedge \frac{i}{2 \pi}(\bar{\partial} \partial)_{M}\left(\frac{\Delta_{V} \psi}{2}\right) .
\end{aligned}
$$

Thus we have

$$
\begin{aligned}
\frac{\mathfrak{C}_{[k]}}{\Omega}(\psi)= & \frac{k^{m}}{k \cdot k} \cdot(-2+m+n) ! \cdot \frac{\Delta_{V}}{4 \pi}\left(\frac{Q_{m}\left(\psi: \phi_{1}\right)}{2 \pi}+Q_{M}(\psi)\right) \\
& +\frac{k^{m}}{k \cdot k} \cdot(-2+m+n) ! \cdot\left(-m \cdot \frac{e(E)}{\omega_{M}}+\frac{\Delta_{M} \phi_{0}}{2}\right) \cdot \frac{\Delta_{V}}{4 \pi}\left(\frac{-\Delta_{M} \psi}{2}\right) \\
& -\frac{k^{m}}{k \cdot k} \cdot \frac{\Lambda_{M}\left[\check{\pi}^{*} \operatorname{trace}\left(\frac{i}{2 \pi} F_{H_{E}}\right)\right]}{n} \cdot(-2+m+n) ! \cdot \frac{\Delta_{V}}{8 \pi} \Delta_{M} \psi \\
& +\frac{k^{m}}{k \cdot k} \cdot(-2+m+n) ! \cdot \frac{\Delta_{M} \phi_{0}}{2} \cdot \frac{\Delta_{V}}{4 \pi}\left(\frac{\Delta_{M} \psi}{2}\right) \\
& +\frac{k^{m}}{k \cdot k} \cdot(-2+m+n) ! \cdot \frac{\Delta_{M}}{4 \pi}\left(-\frac{\Delta_{V} \phi_{1}}{2} \cdot \frac{\Delta_{V} \psi}{2}+Q_{V}\left(\psi: \phi_{1}\right)\right) \\
& +\frac{k^{m}}{k \cdot k} \cdot(-2+m+n) \cdot \frac{\Delta_{V} \phi_{1}}{2} \cdot \frac{\Delta_{M}}{4 \pi}\left(\frac{\Delta_{V} \psi}{2}\right) \\
& +\frac{k^{m}}{k \cdot k} \cdot{ }^{2} D_{V}^{\emptyset}\left(k^{-0}\right) \Delta_{M} \psi+\frac{k^{m}}{k \cdot k} \cdot{ }^{2} D^{\emptyset}\left(k^{-0}\right) \Delta_{V} \psi
\end{aligned}
$$

and, by applying the Einstein-Hermitian condition to the right-hand side of (II.10),

$$
\begin{aligned}
\frac{\widehat{\mathfrak{B}}_{[k]}}{\Omega}(\psi)= & \frac{k^{m}}{k} \cdot \frac{(-2+m+n) !}{8 \pi \cdot n} \cdot \Lambda_{M} \check{\pi}^{*} \operatorname{trace}\left(\frac{i}{2 \pi} F_{H_{E}}\right) \\
& \cdot \Delta_{V} \circ\left(-\Delta_{V} \psi+4 \pi \cdot n \cdot \psi\right) \\
& +\frac{k^{m}}{k} \cdot{ }^{2} D_{V}^{\emptyset}\left(k^{-0}\right)\left(-4 \pi \cdot n \cdot \psi+\Delta_{V} \psi\right) .
\end{aligned}
$$


Since $\nabla^{\mathcal{H}}$ preserves the spaces $\check{\pi}^{*} \Gamma(M: \mathbb{R})$ and $\Gamma(M: W)$ we infer from (II.11) the following results through Lemma I.A and (I.3) respectively:

$$
\begin{aligned}
\hat{\sigma}\left(\frac{\widehat{\mathfrak{I}}_{[k]} \psi}{\Omega}\right)= & \frac{k^{m}}{k \cdot k} \cdot(-2+m+n) ! \cdot \frac{\mathcal{V}_{M} \hat{\sigma}(\psi)}{\Omega_{M}} \\
& +\frac{k^{m}}{k \cdot k} \cdot \hat{\sigma}\left[{ }^{2} D^{\emptyset}\left(k^{-0}\right) \sigma(\psi)\right] \\
& +\frac{k^{m}}{k \cdot k} \cdot \hat{\sigma}\left[{ }^{2} D^{\emptyset}\left(k^{-0}\right) \tilde{\sigma}(\psi)\right]
\end{aligned}
$$

and

$$
\begin{aligned}
\sigma\left(\frac{\widehat{\mathfrak{L}}_{[k]} \psi}{\Omega}\right)= & k^{m} \cdot \frac{(-2+m+n) !}{8 \pi} \cdot \frac{\Delta_{M}}{k}\left(8 \pi \cdot n \cdot \sigma(\psi)+\frac{\Delta_{M}}{k} \sigma(\psi)\right) \\
& +\frac{k^{m}}{k \cdot k} \cdot(-2+m+n) ! \cdot \check{Q}_{M}(\hat{\sigma}(\psi)) \\
& +\frac{k^{m}}{k} \cdot \sigma\left[{ }^{2} D_{V}^{\emptyset}\left(k^{-0}\right) \tilde{\sigma}(\psi)\right]+\frac{k^{m}}{k \cdot k} \cdot \sigma\left[{ }^{2} D^{\emptyset}\left(k^{-0}\right) \sigma(\psi)\right] \\
& +\frac{k^{m}}{k \cdot k} \cdot \sigma\left[{ }^{2} D^{\emptyset}\left(k^{-0}\right) \tilde{\sigma}(\psi)\right] .
\end{aligned}
$$

Let $P_{[k]}$ denote the linear partial differential operator on $\mathbb{P}(E)$ defined as follows:

$$
\begin{array}{r}
P_{[k]} f \equiv\left[\Delta_{V} \circ\left(-4 \pi \cdot n \cdot i d .+\Delta_{V}\right)+\Delta_{V} \circ \frac{\Delta_{M}}{k}\right. \\
\left.+\frac{\Delta_{M}}{k} \circ \Delta_{V}+\frac{\Delta_{M}}{k} \circ \frac{\Delta_{M}}{k}\right] f
\end{array}
$$

$\forall f \in \Gamma(\mathbb{P}(E): \mathbb{R})$. Since $\nabla^{\mathcal{H}}$ preserves the spaces $\check{\pi}^{*} \Gamma(M: \mathbb{R})$ and $\Gamma(M: W)$, from (II.11) we obtain that

$$
\begin{aligned}
\tilde{\sigma}\left(\frac{\widehat{\mathfrak{S}}_{[k]} \psi}{\Omega}\right)= & k^{m} \cdot \frac{(-2+m+n) !}{8 \pi} \cdot P_{[k]} \tilde{\sigma}(\psi) \\
& +\frac{k^{m}}{k} \cdot \frac{(-2+m+n) !}{4 \pi} \\
& \quad \cdot \tilde{\sigma}\left[\Delta_{V} \circ\left(-\frac{\Delta_{V} \phi_{1}}{2} \cdot \frac{\Delta_{V} \sigma(\psi)}{2}+Q_{V}\left(\sigma(\psi): \phi_{1}\right)\right)\right] \\
& +\frac{k^{m}}{k} \cdot \tilde{\sigma}\left[{ }^{4} D_{V}^{\emptyset}\left(k^{-0}\right) \tilde{\sigma}(\psi)\right] \\
& +\frac{k^{m}}{k \cdot k} \cdot \tilde{\sigma}\left[{ }^{2} D^{\emptyset}\left(k^{-0}\right) \sigma(\psi)\right]+\frac{k^{m}}{k \cdot k} \cdot \tilde{\sigma}\left[{ }^{2} D^{\emptyset}\left(k^{-0}\right) \tilde{\sigma}(\psi)\right]
\end{aligned}
$$

through Lemma I.A. Besides we note that

$$
\frac{\widehat{\mathfrak{B}}_{[k]} \psi}{\Omega}=\frac{\widehat{\mathfrak{B}}_{[k]} \tilde{\sigma}(\psi)}{\Omega}=\frac{k^{m}}{k} \cdot{ }^{4} D_{V}^{\emptyset}\left(k^{-0}\right) \tilde{\sigma}(\psi) .
$$


In order to summarize the results established so far we introduce some notation. We use the symbols ${ }^{2} D^{\emptyset}\left(k^{-0}\right) *{ }^{2} D_{V}^{\emptyset}\left(k^{-0}\right)$ and ${ }^{2} D_{V}^{\emptyset}\left(k^{-0}\right) *$ ${ }^{2} D^{\emptyset}\left(k^{-0}\right)$ to indicate respectively the finite sums of the following forms

$$
{ }^{2} D^{\emptyset}\left(k^{-0}\right) \circ{ }^{2} D_{V}^{\emptyset}\left(k^{-0}\right)
$$

and

$$
{ }^{2} D_{V}^{\emptyset}\left(k^{-0}\right) \circ{ }^{2} D^{\emptyset}\left(k^{-0}\right) .
$$

Then, by (II.13), we have

$$
\begin{aligned}
\hat{\sigma}\left(\frac{\mathfrak{c}_{[k]} \psi}{\Omega}\right)= & \frac{k^{m}}{k \cdot k} \cdot \hat{\sigma}\left[{ }^{2} D^{\emptyset}\left(k^{-0}\right) *{ }^{2} D_{V}^{\emptyset}\left(k^{-0}\right) \sigma(\psi)\right] \\
& +\frac{k^{m}}{k \cdot k} \cdot \hat{\sigma}\left[{ }^{2} D^{\emptyset}\left(k^{-0}\right) *{ }^{2} D_{V}^{\emptyset}\left(k^{-0}\right) \tilde{\sigma}(\psi)\right] \\
& +\frac{k^{m}}{k \cdot k} \cdot \hat{\sigma}\left[{ }^{2} D_{V}^{\emptyset}\left(k^{-0}\right) \Delta_{M} \psi\right] \\
& +\frac{k^{m}}{k \cdot k} \cdot \hat{\sigma}\left[{ }^{2} D^{\emptyset}\left(k^{-0}\right) \Delta_{V} \psi\right] .
\end{aligned}
$$

Finally, an application of Lemma I.A to (II.13) yields:

$$
\begin{aligned}
\sigma\left(\frac{\mathfrak{C}_{[k]} \psi}{\Omega}\right)= & \frac{k^{m}}{k \cdot k} \cdot(-2+m+n) ! \cdot \check{Q}_{M}(\hat{\sigma}(\psi)) \\
& +\frac{k^{m}}{k \cdot k} \cdot \sigma\left[{ }^{2} D^{\emptyset}\left(k^{-0}\right) *{ }^{2} D_{V}^{\emptyset}\left(k^{-0}\right) \sigma(\psi)\right] \\
& +\frac{k^{m}}{k \cdot k} \cdot \sigma\left[{ }^{2} D^{\emptyset}\left(k^{-0}\right) \sigma(\psi)\right] \\
& +\frac{k^{m}}{k \cdot k} \cdot \sigma\left[{ }^{2} D^{\emptyset}\left(k^{-0}\right) \tilde{\sigma}(\psi)\right] \\
& +\frac{k^{m}}{k \cdot k} \cdot \sigma\left[{ }^{2} D^{\emptyset}\left(k^{-0}\right) *{ }^{2} D_{V}^{\emptyset}\left(k^{-0}\right) \tilde{\sigma}(\psi)\right] \\
& +\frac{k^{m}}{k \cdot k} \cdot \sigma\left[{ }^{2} D_{V}^{\emptyset}\left(k^{-0}\right) \Delta_{M} \psi\right] \\
& +\frac{k^{m}}{k \cdot k} \cdot \sigma\left[{ }^{2} D^{\emptyset}\left(k^{-0}\right) \Delta_{V} \psi\right]
\end{aligned}
$$

and

$$
\begin{aligned}
\tilde{\sigma}\left(\frac{\mathfrak{c}_{[k]} \psi}{\Omega}\right)= & \frac{k^{m}}{k \cdot k} \cdot \tilde{\sigma}\left[{ }^{2} D_{V}^{\emptyset}\left(k^{-0}\right) *{ }^{2} D^{\emptyset}\left(k^{-0}\right) \sigma(\psi)\right] \\
& +\frac{k^{m}}{k \cdot k} \cdot \tilde{\sigma}\left[{ }^{2} D_{V}^{\emptyset}\left(k^{-0}\right) *{ }^{2} D^{\emptyset}\left(k^{-0}\right) \tilde{\sigma}(\psi)\right] \\
& +\frac{k^{m}}{k \cdot k} \cdot \tilde{\sigma}\left[{ }^{2} D^{\emptyset}\left(k^{-0}\right) *{ }^{2} D_{V}^{\emptyset}\left(k^{-0}\right) \sigma(\psi)\right] \\
& +\frac{k^{m}}{k \cdot k} \cdot \tilde{\sigma}\left[{ }^{2} D^{\emptyset}\left(k^{-0}\right) *{ }^{2} D_{V}^{\emptyset}\left(k^{-0}\right) \tilde{\sigma}(\psi)\right] \\
& +\frac{k^{m}}{k \cdot k} \cdot \tilde{\sigma}\left[{ }^{2} D_{V}^{\emptyset}\left(k^{-0}\right) \Delta_{M} \psi\right] \\
& +\frac{k^{m}}{k \cdot k} \cdot \tilde{\sigma}\left[{ }^{2} D^{\emptyset}\left(k^{-0}\right) \Delta_{V} \psi\right] .
\end{aligned}
$$


We can now summarize these results established so far in the following

Proposition II.A. Assume that $\psi \in \Gamma(\mathbb{P}(E): \mathbb{R})$. Let

$$
\mathbf{P}_{[k]} \equiv \frac{\widehat{\mathfrak{L}}_{[k]}}{\Omega}+\frac{\widehat{\mathfrak{B}}_{[k]}}{\Omega}+\frac{\mathfrak{C}_{[k]}}{\Omega} .
$$

Then we have

$$
\begin{aligned}
\sigma\left(\mathbf{P}_{[k]} \psi\right)= & k^{m} \cdot \frac{(-2+m+n) !}{8 \pi} \cdot \frac{\Delta_{M}}{k}\left(8 \pi \cdot n \cdot \sigma(\psi)+\frac{\Delta_{M}}{k} \sigma(\psi)\right) \\
& +\frac{k^{m}}{k \cdot k} \cdot 2 \cdot(-2+m+n) ! \cdot \check{Q}_{M}(\hat{\sigma}(\psi)) \\
& +\frac{k^{m}}{k} \cdot \sigma\left[{ }^{2} D_{V}^{\emptyset}\left(k^{-0}\right) \tilde{\sigma}(\psi)\right] \\
& +\frac{k^{m}}{k \cdot k} \cdot \sigma\left[{ }^{2} D^{\emptyset}\left(k^{-0}\right) \sigma(\psi)\right] \\
& +\frac{k^{m}}{k \cdot k} \cdot \sigma\left[{ }^{2} D^{\emptyset}\left(k^{-0}\right) \tilde{\sigma}(\psi)\right] \\
& +\frac{k^{m}}{k} \cdot \sigma\left[{ }^{4} D_{V}^{\emptyset}\left(k^{-0}\right) \tilde{\sigma}(\psi)\right] \\
& +\frac{k^{m}}{k \cdot k} \cdot \sigma\left[{ }^{2} D^{\emptyset}\left(k^{-0}\right) *{ }^{2} D_{V}^{\emptyset}\left(k^{-0}\right) \tilde{\sigma}(\psi)\right] \\
& +\frac{k^{m}}{k \cdot k} \cdot \sigma\left[{ }^{2} D_{V}^{\emptyset}\left(k^{-0}\right) \Delta_{M} \psi\right] \\
& +\frac{k^{m}}{k \cdot k} \cdot \sigma\left[{ }^{2} D^{\emptyset}\left(k^{-0}\right) \Delta_{V} \psi\right]
\end{aligned}
$$

and

$$
\begin{aligned}
\tilde{\sigma}\left(\mathbf{P}_{[k]} \psi\right)= & k^{m} \cdot \frac{(-2+m+n) !}{8 \pi} \cdot P_{[k]} \tilde{\sigma}(\psi) \\
& +\frac{k^{m}}{k} \cdot \frac{(-2+m+n) !}{4 \pi} \\
& \cdot \tilde{\sigma}\left[\Delta_{V} \circ\left(-\frac{\Delta_{V} \phi_{1}}{2} \cdot \frac{\Delta_{V} \sigma(\psi)}{2}+Q_{V}\left(\sigma(\psi): \phi_{1}\right)\right)\right] \\
& +\frac{k^{m}}{k} \cdot \tilde{\sigma}\left[{ }^{4} D_{V}^{\emptyset}\left(k^{-0}\right) \tilde{\sigma}(\psi)\right] \\
& +\frac{k^{m}}{k \cdot k} \cdot \tilde{\sigma}\left[{ }^{2} D^{\emptyset}\left(k^{-0}\right) \sigma(\psi)\right]+\frac{k^{m}}{k \cdot k} \cdot \tilde{\sigma}\left[{ }^{2} D^{\emptyset}\left(k^{-0}\right) \tilde{\sigma}(\psi)\right] \\
& +\frac{k^{m}}{k \cdot k} \cdot \tilde{\sigma}\left[{ }^{2} D_{V}^{\emptyset}\left(k^{-0}\right) *{ }^{2} D^{\emptyset}\left(k^{-0}\right) \sigma(\psi)\right] \\
& +\frac{k^{m}}{k \cdot k} \cdot \tilde{\sigma}\left[{ }^{2} D_{V}^{\emptyset}\left(k^{-0}\right) *{ }^{2} D^{\emptyset}\left(k^{-0}\right) \tilde{\sigma}(\psi)\right] \\
& +\frac{k^{m}}{k \cdot k} \cdot \tilde{\sigma}\left[{ }^{2} D^{\emptyset}\left(k^{-0}\right) *{ }^{2} D_{V}^{\emptyset}\left(k^{-0}\right) \sigma(\psi)\right] \\
& +\frac{k^{m}}{k \cdot k} \cdot \tilde{\sigma}\left[{ }^{2} D^{\emptyset}\left(k^{-0}\right) *{ }^{2} D_{V}^{\emptyset}\left(k^{-0}\right) \tilde{\sigma}(\psi)\right] \\
& +\frac{k^{m}}{k \cdot k} \cdot \tilde{\sigma}\left[{ }^{2} D_{V}^{\emptyset}\left(k^{-0}\right) \Delta_{M} \psi\right] \\
& +\frac{k^{m}}{k \cdot k} \cdot \tilde{\sigma}\left[{ }^{2} D^{\emptyset}\left(k^{-0}\right) \Delta_{V} \psi\right] .
\end{aligned}
$$


Moreover,

$$
\begin{aligned}
\hat{\sigma}\left(\mathbf{P}_{[k]} \psi\right)= & \frac{k^{m}}{k \cdot k} \cdot(-2+m+n) ! \cdot \frac{\mathcal{V}_{M} \hat{\sigma}(\psi)}{\Omega_{M}} \\
& +\frac{k^{m}}{k \cdot k} \cdot \hat{\sigma}\left[{ }^{2} D^{\emptyset}\left(k^{-0}\right) \sigma(\psi)\right] \\
& +\frac{k^{m}}{k \cdot k} \cdot \hat{\sigma}\left[{ }^{2} D^{\emptyset}\left(k^{-0}\right) \tilde{\sigma}(\psi)\right] \\
& +\frac{k^{m}}{k} \cdot \hat{\sigma}\left[{ }^{4} D_{V}^{\emptyset}\left(k^{-0}\right) \tilde{\sigma}(\psi)\right] \\
& +\frac{k^{m}}{k \cdot k} \cdot \hat{\sigma}\left[{ }^{2} D^{\emptyset}\left(k^{-0}\right) *{ }^{2} D_{V}^{\emptyset}\left(k^{-0}\right) \sigma(\psi)\right] \\
& +\frac{k^{m}}{k \cdot k} \cdot \hat{\sigma}\left[{ }^{2} D^{\emptyset}\left(k^{-0}\right) *{ }^{2} D_{V}^{\emptyset}\left(k^{-0}\right) \tilde{\sigma}(\psi)\right] \\
& +\frac{k^{m}}{k \cdot k} \cdot \hat{\sigma}\left[{ }^{2} D_{V}^{\emptyset}\left(k^{-0}\right) \Delta_{M} \psi\right] \\
& +\frac{k^{m}}{k \cdot k} \cdot \hat{\sigma}\left[{ }^{2} D^{\emptyset}\left(k^{-0}\right) \Delta_{V} \psi\right] .
\end{aligned}
$$

Here $P_{[k]}$ is the linear partial differential operator on $\mathbb{P}(E)$ defined as follows:

$P_{[k]} f \equiv\left[\Delta_{V} \circ\left(-4 \pi \cdot n \cdot i d .+\Delta_{V}\right)+\Delta_{V} \circ \frac{\Delta_{M}}{k}+\frac{\Delta_{M}}{k} \circ \Delta_{V}+\frac{\Delta_{M}}{k} \circ \frac{\Delta_{M}}{k}\right] f$

$\forall f \in \Gamma(\mathbb{P}(E): \mathbb{R})$.

\section{Estimates}

Our goal in this section is to establish apriori estimates (Proposition III.C and Corollary III.C) for $\psi$ through fundamental results of the theory of elliptic linear partial differential equations. Our derivation is based on the effective expression of $\frac{{ }_{N} \mathfrak{S}_{[k]}}{\Omega}$ established in the last section: Proposition II.A and (II.12).

Convention. In this section $C$ will always be used to indicate a positive constant independent of $k \in \mathbb{N}$.

Let ${ }^{V} \nabla$ and ${ }^{M} \nabla$ be respectively the fiber-directional component and the horizontal component of $\nabla$ so that

$$
\nabla={ }^{V} \nabla \oplus{ }^{M} \nabla
$$

We define ${ }_{[k]}^{M} \nabla$ and ${ }_{[k]} \nabla$ as follows:

$$
\stackrel{M}{[k]} \nabla \equiv \frac{{ }^{M} \nabla}{\sqrt{k}}
$$


and

$$
{ }_{[k]} \nabla \equiv{ }^{V} \nabla \oplus \frac{{ }^{M} \nabla}{\sqrt{k}}={ }^{V} \nabla \oplus{ }_{[k]}^{M} \nabla .
$$

Proposition III.A. Let $P_{[k]}$ denote the linear partial differential operator on $\mathbb{P}(E)$ defined as follows:

$$
\begin{aligned}
& P_{[k]} \psi \\
& \equiv\left[\Delta_{V} \circ\left(-4 \pi \cdot n \cdot i d .+\Delta_{V}\right)+\Delta_{V} \circ \frac{\Delta_{M}}{k}+\frac{\Delta_{M}}{k} \circ \Delta_{V}+\frac{\Delta_{M}}{k} \circ \frac{\Delta_{M}}{k}\right] \psi
\end{aligned}
$$

$\forall \psi \in \Gamma(\mathbb{P}(E): \mathbb{R})$. Then

$$
\begin{aligned}
\frac{\|\sigma(\psi)\|}{k}+ & \frac{\left\|{ }^{M} \nabla \sigma(\psi)\right\|}{k}+\frac{{ }^{M} \nabla \circ{ }^{M} \nabla \sigma(\psi) \|}{k} \\
+ & \|\|_{[k]}^{M} \nabla \circ{ }_{[k]}^{M} \nabla \circ{ }_{[k]}^{M} \nabla \sigma(\psi) \| \\
+ & \left\|{ }_{[k]}^{M} \nabla \circ{ }_{[k]}^{M} \nabla \circ{ }_{[k]}^{M} \nabla \circ{ }_{[k]}^{M} \nabla \sigma(\psi)\right\| \\
& \leq C \cdot\left\|\sigma\left(P_{[k]} \psi\right)\right\|
\end{aligned}
$$

and

$$
\begin{aligned}
\|\tilde{\sigma}(\psi)\| & +\left\|_{[k]} \nabla \tilde{\sigma}(\psi)\right\|+\left\|_{[k]} \nabla \circ{ }_{[k]} \nabla \tilde{\sigma}(\psi)\right\| \\
& +\left\|_{[k]} \nabla \circ{ }_{[k]} \nabla \circ{ }_{[k]} \nabla \tilde{\sigma}(\psi)\right\| \\
& +\left\|_{[k]} \nabla \circ{ }_{[k]} \nabla \circ{ }_{[k]} \nabla \circ{ }_{[k]} \nabla \tilde{\sigma}(\psi)\right\| \\
& \leq C \cdot\left\|\tilde{\sigma}\left(P_{[k]} \psi\right)\right\| .
\end{aligned}
$$

Besides for $\psi \in \Gamma(\mathbb{P}(E): \mathbb{R})$ satisfying the normalization condition

$$
\int_{\mathbb{P}(E)} \psi \cdot \Omega=0 \Longleftrightarrow \int_{\mathbb{P}(E)} \hat{\sigma}(\psi) \cdot \Omega=0
$$

we have the following estimate

$$
\begin{aligned}
\|\hat{\sigma}(\psi)\| & +\left\|{ }^{M} \nabla \hat{\sigma}(\psi)\right\|+\left\|{ }^{M} \nabla \circ{ }^{M} \nabla \hat{\sigma}(\psi)\right\| \\
& +\left\|{ }^{M} \nabla \circ{ }^{M} \nabla \circ{ }^{M} \nabla \hat{\sigma}(\psi)\right\| \\
& +\left\|{ }^{M} \nabla \circ{ }^{M} \nabla \circ{ }^{M} \nabla \circ{ }^{M} \nabla \hat{\sigma}(\psi)\right\| \\
& \leq C \cdot\left\|\frac{\mathcal{V}_{M}}{\Omega_{M}} \hat{\sigma}(\psi)\right\| .
\end{aligned}
$$

Here

$$
\begin{aligned}
\mathcal{V}_{M} \bullet \equiv & \frac{\omega_{M}^{(-1+m)}}{(-1+m) !} \wedge i(\bar{\partial} \partial)_{M} \frac{\Delta_{M} \bullet}{4 \pi}+m \cdot \frac{e\left(K_{M}\right)}{\omega_{M}} \cdot \frac{\omega_{M}^{(-1+m)}}{(-1+m) !} \wedge i(\bar{\partial} \partial)_{M} \bullet \\
& +\frac{(-1+m) \cdot \omega_{M}^{(-2+m)}}{(-1+m) !} \wedge i(\bar{\partial} \partial)_{M} \bullet \wedge \check{\pi}^{*} \operatorname{trace}\left(\frac{i}{2 \pi} F_{H_{M}}\right)
\end{aligned}
$$


is the infinitesimal deformation operator for the constant Hermitian scalar curvature equation on $\left(M: \omega_{M}\right)$.

Proof. (III.3) follows from standard theory of elliptic linear partial differential equations.

Note that $\Delta_{V}$ and $\Delta_{M}$ preserve the decomposition $i d .=\hat{\sigma} \oplus \sigma \oplus \tilde{\sigma}$ :

$$
\Delta_{V} \circ \hat{\sigma} \oplus \Delta_{V} \circ \sigma \oplus \Delta_{V} \circ \tilde{\sigma}=\hat{\sigma} \circ \Delta_{V} \oplus \sigma \circ \Delta_{V} \oplus \tilde{\sigma} \circ \Delta_{V}
$$

and

$$
\Delta_{M} \circ \hat{\sigma} \oplus \Delta_{M} \circ \sigma \oplus \Delta_{M} \circ \tilde{\sigma}=\hat{\sigma} \circ \Delta_{M} \oplus \sigma \circ \Delta_{M} \oplus \tilde{\sigma} \circ \Delta_{M} .
$$

We will use these results implicitly in the following derivation of (III.1) and (III.2).

To show that (III.1) is true we note that

$$
\sigma\left(P_{[k]} \psi\right)=\left(8 \pi \cdot n \cdot \sigma(\psi)+\frac{\Delta_{M}}{k}\right) \circ \frac{\Delta_{M}}{k} \sigma(\psi) .
$$

Standard theory of elliptic linear partial differential operators shows that

$$
\frac{\|s\|}{k}+\frac{\Perp^{M} \nabla s \|}{k}+\|\left[\begin{array}{l}
M \\
{[k]}
\end{array} \nabla \circ{ }_{[k]}^{M} \nabla s\|\leq C \cdot\| \frac{\Delta_{M}}{k} s \|\right.
$$

$\forall s \in \Gamma(M: W)$. On the other hand, by the Stokes' Theorem we have

$$
\begin{aligned}
<\left(8 \pi n \cdot i d .+\frac{\Delta_{M}}{k}\right) s: s> & =8 \pi n \cdot\langle s: s> \\
& +<{ }_{[k]}^{M} \nabla s:{ }_{[k]}^{M} \nabla s>
\end{aligned}
$$

and thence, by the Schwarz inequality, we have

$$
\|s\|+\left\|\begin{array}{l}
M \\
{[k]}
\end{array} \nabla s\right\| \leq C \cdot\left\|\left(8 \pi \cdot n \cdot i d .+\frac{\Delta_{M}}{k}\right) s\right\|
$$

$\forall s \in \Gamma(M: W)$. Now using the Stokes' Theorem again we obtain

$$
\begin{aligned}
<{ }_{[k]}^{M} \nabla \circ{ }_{[k]}^{M} \nabla s:{ }_{[k]}^{M} \nabla \circ{ }_{[k]}^{M} \nabla s>= & <{ }_{[k]}^{M} \nabla s:{ }_{[k]}^{M} \nabla^{*} \circ{ }_{[k]}^{M} \nabla \circ{ }_{[k]}^{M} \nabla s> \\
= & <{ }_{[k]}^{M} \nabla s:{ }_{[k]}^{M} \nabla \circ{ }_{[k]}^{M} \nabla^{*} \circ{ }_{[k]}^{M} \nabla s> \\
& \left.+<{ }_{[k]}^{M} \nabla s:{ }_{[k]}^{M} \nabla^{*}:{ }_{[k]}^{M} \nabla\right] \circ{ }_{[k]}^{M} \nabla s> \\
= & <{ }_{[k]}^{M} \nabla^{*} \circ{ }_{[k]}^{M} \nabla s:{ }_{[k]}^{M} \nabla^{*} \circ{ }_{[k]}^{M} \nabla s> \\
& +<{ }_{[k]}^{M} \nabla s:\left[{ }_{[k]}^{M} \nabla^{*}:{ }_{[k]}^{M} \nabla\right] \circ{ }_{[k]}^{M} \nabla s>
\end{aligned}
$$


$\forall s \in \Gamma(M: W)$. Here ${ }_{[k]}^{M} \nabla^{*}$ is the adjoint of ${ }_{[k]}^{M} \nabla$ with respect to the Hermitian form (metric) $\check{\omega}$ on $\mathbb{P}(E)$. Thus, by the Schwarz inequality,

$$
\begin{aligned}
<{ }_{[k]}^{M} \nabla \circ{ }_{[k]}^{M} \nabla s:{ }_{[k]}^{M} \nabla \circ{ }_{[k]}^{M} \nabla s> & \leq C \cdot<\frac{\Delta_{M}}{k} s: \frac{\Delta_{M}}{k} s>+C \cdot\left\|{ }_{[k]}^{M} \nabla s\right\|^{2} \\
& \leq C \cdot\left\|\left(8 \pi \cdot n \cdot i d \cdot+\frac{\Delta_{M}}{k}\right) s\right\|^{2} \\
& +C \cdot\|s\|^{2}+C \cdot\left\|\frac{M}{[k]} \nabla s\right\|^{2} \\
& \leq C \cdot\left\|\left(8 \pi \cdot n \cdot i d \cdot+\frac{\Delta_{M}}{k}\right) s\right\|^{2}
\end{aligned}
$$

and thence

$$
\|s\|+\left\|{ }_{[k]}^{M} \nabla s\right\|+\left\|{ }_{[k]}^{M} \nabla \circ{ }_{[k]}^{M} \nabla s\right\| \leq C \cdot\left\|\left(8 \pi \cdot n \cdot i d .+\frac{\Delta_{M}}{k}\right) s\right\|
$$

$\forall s \in \Gamma(M: W)$. Using (III.5) and (III.10) we then obtain (III.1) via (III.4).

Now we establish (III.2) through the same process as above. We will use the following inequalities:

$$
\|\tilde{\sigma}(f)\|^{2} \leq C \cdot\left\langle{ }^{V} \nabla \tilde{\sigma}(f):{ }^{V} \nabla \tilde{\sigma}(f)>\right.
$$

and

$$
\|\tilde{\sigma}(f)\|^{2}+\left\|{ }^{V} \nabla \tilde{\sigma}(f)\right\|^{2} \leq C \cdot<\left(-4 \pi n \cdot i d .+\Delta_{V}\right) \tilde{\sigma}(f): \tilde{\sigma}(f)>.
$$

By the Stokes' Theorem we have

$$
\begin{aligned}
<\left(\Delta_{V}+\frac{\Delta_{M}}{k}\right) \tilde{\sigma}(f): \tilde{\sigma}(f)>= & <{ }^{V} \nabla \tilde{\sigma}(f):{ }^{V} \nabla \tilde{\sigma}(f)> \\
& +<{ }_{[k]}^{M} \nabla \tilde{\sigma}(f):{ }_{[k]}^{M} \nabla \tilde{\sigma}(f)>
\end{aligned}
$$

and

$$
\begin{aligned}
<[(-4 \pi n \cdot i d . & \left.\left.+\Delta_{V}\right)+\frac{\Delta_{M}}{k}\right] \tilde{\sigma}(f): \tilde{\sigma}(f)> \\
= & <\left(-4 \pi n \cdot i d .+\Delta_{V}\right) \tilde{\sigma}(f): \tilde{\sigma}(f)> \\
& +{ }_{[k]}^{M} \nabla \tilde{\sigma}(f):{ }_{[k]}^{M} \nabla \tilde{\sigma}(f)>
\end{aligned}
$$

$\forall f \in \Gamma(\mathbb{P}(E): \mathbb{R})$. Thus, using of the Schwarz inequality yields

$$
\begin{aligned}
\|\tilde{\sigma}(f)\|+\left\|{ }^{V} \nabla \circ \tilde{\sigma}(f)\right\| & +\left\|{ }_{[k]}^{M} \nabla \tilde{\sigma}(f)\right\| \\
& \leq C \cdot\left\|\left(\Delta_{V}+\frac{\Delta_{M}}{k}\right) \tilde{\sigma}(f)\right\|
\end{aligned}
$$


and

$$
\begin{aligned}
\|\tilde{\sigma}(f)\|+\left\|^{V} \nabla \tilde{\sigma}(f)\right\|+\|\underset{[k]}{M} \nabla \tilde{\sigma}(f)\| \\
\leq C \cdot\left\|\left[\left(-4 \pi n \cdot i d .+\Delta_{V}\right)+\frac{\Delta_{M}}{k}\right] \tilde{\sigma}(f)\right\|
\end{aligned}
$$

$\forall f \in \Gamma(\mathbb{P}(E): \mathbb{R})$. Now by the Stokes' Theorem again we obtain

$$
\begin{aligned}
& <_{[k]} \nabla \circ_{[k]} \nabla \tilde{\sigma}(f):{ }_{[k]} \nabla \circ_{[k]} \nabla \tilde{\sigma}(f)> \\
& =<_{[k]} \nabla \tilde{\sigma}(f):_{[k]} \nabla^{*}{ }_{[k]} \nabla \circ{ }_{[k]} \nabla \tilde{\sigma}(f)> \\
& =<{ }_{[k]} \nabla \tilde{\sigma}(f):_{[k]} \nabla \circ_{[k]} \nabla^{*} \circ_{[k]} \nabla \tilde{\sigma}(f)> \\
& +<{ }_{[k]} \nabla \tilde{\sigma}(f):\left[_{[k]} \nabla^{*}:{ }_{[k]} \nabla\right] \circ{ }_{[k]} \nabla \tilde{\sigma}(f)> \\
& =<{ }_{[k]} \nabla^{*} o_{[k]} \nabla \tilde{\sigma}(f):{ }_{[k]} \nabla^{*} o_{[k]} \nabla \tilde{\sigma}(f)> \\
& +<{ }_{[k]} \nabla \tilde{\sigma}(f):\left[{ }_{[k]} \nabla^{*}:{ }_{[k]} \nabla\right] \circ{ }_{[k]} \nabla \tilde{\sigma}(f)>
\end{aligned}
$$

$\forall f \in \Gamma(\mathbb{P}(E): \mathbb{R})$. Here ${ }_{[k]} \nabla^{*}$ is the adjoint of ${ }_{[k]} \nabla$ with respect to the Hermitian form (metric) $\breve{\omega}$ on $\mathbb{P}(E)$. Thus by the Schwarz inequality we have

$$
\begin{gathered}
<_{[k]} \nabla \circ{ }_{[k]} \nabla \tilde{\sigma}(f):_{[k]} \nabla{ }_{[k]} \nabla \tilde{\sigma}(f)> \\
\leq C \cdot\left\|\left(\Delta_{V}+\frac{\Delta_{M}}{k}\right) \tilde{\sigma}(f)\right\|^{2} \\
+C \cdot\left\|_{[k]} \nabla \tilde{\sigma}(f)\right\|^{2} .
\end{gathered}
$$

Hence, using (III.13) and (III.14), we get

$$
\begin{aligned}
\|\tilde{\sigma}(f)\|+\left\|_{[k]} \nabla \tilde{\sigma}(f)\right\|+\left\|_{[k]} \nabla{ }_{[k]} \nabla \tilde{\sigma}(f)\right\| \\
\leq C \cdot\left\|\left(\Delta_{V}+\frac{\Delta_{M}}{k}\right) \tilde{\sigma}(f)\right\|
\end{aligned}
$$

and

$$
\|\tilde{\sigma}(f)\|+\left\|_{[k]} \nabla \tilde{\sigma}(f)\right\|+\left\|_{[k]} \nabla \circ[k] \nabla \tilde{\sigma}(f)\right\|
$$

$$
\leq C \cdot\left\|\left[\left(-4 \pi \cdot n \cdot i d .+\Delta_{V}\right)+\frac{\Delta_{M}}{k}\right] \tilde{\sigma}(f)\right\|
$$

$\forall f \in \Gamma(\mathbb{P}(E): \mathbb{R})$. Since

$$
\begin{aligned}
\tilde{\sigma}\left(P_{[k]} \psi\right) & \\
= & {\left[\Delta_{V} \circ\left(-4 \pi \cdot n \cdot i d .+\Delta_{V}\right)+\Delta_{V} \frac{\Delta_{M}}{k}+\frac{\Delta_{M}}{k} \Delta_{V}+\frac{\Delta_{M}}{k} \frac{\Delta_{M}}{k}\right] \tilde{\sigma}(\psi) } \\
= & {\left[\left(-4 \pi \cdot n \cdot i d .+\Delta_{V}\right)+\frac{\Delta_{M}}{k}\right] \circ\left[\Delta_{V}+\frac{\Delta_{M}}{k}\right] \tilde{\sigma}(\psi) } \\
& +4 \pi \cdot n \cdot \frac{\Delta_{M}}{k} \tilde{\sigma}(\psi),
\end{aligned}
$$


we deduce from (III.17) and (III.18) that

$$
\begin{aligned}
\|\tilde{\sigma}(\psi)\| & +\left\|_{[k]} \nabla \tilde{\sigma}(\psi)\right\|+\left\|_{[k]} \nabla \circ{ }_{[k]} \nabla \tilde{\sigma}(\psi)\right\| \\
& +\left\|_{[k]} \nabla \circ{ }_{[k]} \nabla \circ{ }_{[k]} \nabla \tilde{\sigma}(\psi)\right\| \\
& +\left\|_{[k]} \nabla \circ{ }_{[k]} \nabla \circ{ }_{[k]} \nabla \circ{ }_{[k]} \nabla \tilde{\sigma}(\psi)\right\| \\
& \leq C \cdot\left\|\tilde{\sigma}\left(P_{[k]} \psi\right)\right\|+C \cdot\left\|\frac{\Delta_{M}}{k} \tilde{\sigma}(\psi)\right\|
\end{aligned}
$$

$\forall \psi \in \Gamma(\mathbb{P}(E): \mathbb{R})$. Thus (III.2) is true once we have shown that

$$
\left\|\frac{\Delta_{M}}{k} \tilde{\sigma}(\psi)\right\| \leq C \cdot\left\|\tilde{\sigma}\left(P_{[k]} \psi\right)\right\|
$$

But this follows from the following result:

$$
\begin{aligned}
&<\tilde{\sigma}\left(P_{[k]} \psi\right): \Delta_{V} \tilde{\sigma}(\psi)> \\
&=<\left(-4 \pi \cdot n \cdot i d .+\Delta_{V}\right) \circ \Delta_{V} \tilde{\sigma}(\psi): \Delta_{V} \tilde{\sigma}(\psi)> \\
&+<{ }^{M} \nabla+\circ \Delta_{V} \tilde{\sigma}(\psi):{ }_{[k]}^{M} \nabla \circ \Delta_{V} \tilde{\sigma}(\psi)> \\
&+<{ }^{M} \nabla+\circ \Delta_{V} \tilde{\sigma}(\psi):{ }_{[k]}^{M} \nabla \circ \Delta_{V} \tilde{\sigma}(\psi)> \\
&+<{ }^{V} \nabla \circ \frac{\Delta_{M}}{k} \tilde{\sigma}(\psi):{ }^{V} \nabla \circ \frac{\Delta_{M}}{k} \tilde{\sigma}(\psi)> \\
& \geq<\left(-4 \pi \cdot n \cdot i d .+\Delta_{V}\right) \circ \Delta_{V} \tilde{\sigma}(\psi): \Delta_{V} \tilde{\sigma}(\psi)> \\
&+<{ }^{V} \nabla \circ \frac{\Delta_{M}}{k} \tilde{\sigma}(\psi):{ }^{V} \nabla \circ \frac{\Delta_{M}}{k} \tilde{\sigma}(\psi)>
\end{aligned}
$$

and the Schwarz inequality. q.e.d.

By standard theory of elliptic linear partial differential operators and Proposition III.A we have the following obvious result.

Corollary III.A. Let $P_{[k]}$ denote the linear partial differential operator on $\mathbb{P}(E)$ defined as follows:

$$
\begin{aligned}
& P_{[k]} \psi \\
& \equiv\left[\Delta_{V} \circ\left(-4 \pi \cdot n \cdot i d .+\Delta_{V}\right)+\Delta_{V} \circ \frac{\Delta_{M}}{k}+\frac{\Delta_{M}}{k} \circ \Delta_{V}+\frac{\Delta_{M}}{k} \circ \frac{\Delta_{M}}{k}\right] \psi
\end{aligned}
$$


$\forall \psi \in \Gamma(\mathbb{P}(E): \mathbb{R})$. Then for $\gamma \in \mathbb{N}$ we have the following estimates:

$$
\begin{aligned}
\frac{1}{k}\left\|\sigma\left[\left(\Delta_{V}+\Delta_{M}\right)^{\gamma} \psi\right]\right\| & +\frac{1}{k}\left\|^{M} \nabla \sigma\left[\left(\Delta_{V}+\Delta_{M}\right)^{\gamma} \psi\right]\right\| \\
& +\frac{1}{k}\left\|^{M} \nabla \circ{ }^{M} \nabla \sigma\left[\left(\Delta_{V}+\Delta_{M}\right)^{\gamma} \psi\right]\right\| \\
& +\|_{[k]}^{M} \nabla \circ{ }_{[k]}^{M} \nabla \circ{ }_{[k]}^{M} \nabla \sigma\left[\left(\Delta_{V}+\Delta_{M}\right)^{\gamma} \psi\right] \\
& +\left\|{ }_{[k]}^{M} \nabla \circ{ }_{[k]}^{M} \nabla \circ{ }_{[k]}^{M} \nabla \circ{ }_{[k]}^{M} \nabla \sigma\left[\left(\Delta_{V}+\Delta_{M}\right)^{\gamma} \psi\right]\right\| \\
& \leq C^{[\gamma]} \cdot\left\|\sigma\left[\left(\Delta_{V}+\Delta_{M}\right)^{\gamma} \circ P_{[k]} \psi\right]\right\|
\end{aligned}
$$

and

$$
\begin{aligned}
& \left\|\tilde{\sigma}\left[\left(\Delta_{V}+\Delta_{M}\right)^{\gamma} \psi\right]\right\|+\left\|[k] \nabla \tilde{\sigma}\left[\left(\Delta_{V}+\Delta_{M}\right)^{\gamma} \psi\right]\right\| \\
& +\left\|_{[k]} \nabla \circ_{[k]} \nabla \tilde{\sigma}\left[\left(\Delta_{V}+\Delta_{M}\right)^{\gamma} \psi\right]\right\| \\
& +\left\|_{[k]} \nabla \circ_{[k]} \nabla \circ_{[k]} \nabla \tilde{\sigma}\left[\left(\Delta_{V}+\Delta_{M}\right)^{\gamma} \psi\right]\right\| \\
& +\left\|{ }_{[k]} \nabla \circ_{[k]} \nabla \circ_{[k]} \nabla o_{[k]} \nabla \tilde{\sigma}\left[\left(\Delta_{V}+\Delta_{M}\right)^{\gamma} \psi\right]\right\| \\
& \leq C^{[\gamma]} \cdot\left\|\tilde{\sigma}\left[\left(\Delta_{V}+\Delta_{M}\right)^{\gamma} \circ P_{[k]} \psi\right]\right\| .
\end{aligned}
$$

Besides for $\psi \in \Gamma(\mathbb{P}(E): \mathbb{R})$ satisfying the normalization condition

$$
\int_{\mathbb{P}(E)} \psi \cdot \Omega=0 \Longleftrightarrow \int_{\mathbb{P}(E)} \hat{\sigma}(\psi) \cdot \Omega=0
$$

we have the following estimate:

$$
\begin{aligned}
\left\|\hat{\sigma}\left[\Delta_{M}^{\gamma} \psi\right]\right\| & +\left\|{ }^{M} \nabla \hat{\sigma}\left[\Delta_{M}^{\gamma} \psi\right]\right\|+\left\|{ }^{M} \nabla \circ{ }^{M} \nabla \hat{\sigma}\left[\Delta_{M}^{\gamma} \psi\right]\right\| \\
& +\left\|{ }^{M} \nabla \circ{ }^{M} \nabla \circ{ }^{M} \nabla \hat{\sigma}\left[\Delta_{M}^{\gamma} \psi\right]\right\| \\
& +\left\|{ }^{M} \nabla \circ{ }^{M} \nabla \circ{ }^{M} \nabla \circ{ }^{M} \nabla \hat{\sigma}\left[\Delta_{M}^{\gamma} \psi\right]\right\| \\
& \leq C^{[\gamma]} .\left\|\Delta_{M}^{\gamma} \circ \frac{V_{M}}{\Omega_{M}} \hat{\sigma}(\psi)\right\| .
\end{aligned}
$$

Here

$$
\begin{aligned}
\mathcal{V}_{M} \bullet \equiv & \frac{\omega_{M}^{(-1+m)}}{(-1+m) !} \wedge i(\bar{\partial} \partial)_{M} \frac{\Delta_{M} \bullet}{4 \pi}+m \cdot \frac{e\left(K_{M}\right)}{\omega_{M}} \cdot \frac{\omega_{M}^{(-1+m)}}{(-1+m) !} \wedge i(\bar{\partial} \partial)_{M} \bullet \\
& +\frac{(-1+m) \cdot \omega_{M}^{(-2+m)}}{(-1+m) !} \wedge i(\bar{\partial} \partial)_{M} \bullet \wedge \check{\pi}^{*} \operatorname{trace}\left(\frac{i}{2 \pi} F_{H_{M}}\right)
\end{aligned}
$$

is the infinitesimal deformation operator for the constant Hermitian scalar curvature equation on $\left(M: \omega_{M}\right)$. 
Now we derive estimates for the elliptic linear partial differential operator

$$
\mathbf{P}_{[k]}=\frac{\widehat{\mathfrak{L}}_{[k]}}{\Omega}+\frac{\widehat{\mathfrak{B}}_{[k]}}{\Omega}+\frac{\mathfrak{C}_{[k]}}{\Omega}
$$

based on Proposition II.A and Proposition III.A. We have the following result.

Proposition III.B. Assume that $\psi \in \Gamma(\mathbb{P}(E): \mathbb{R})$ satisfies the normalization condition

$$
\int_{\mathbb{P}(E)} \psi \cdot \Omega=0 \Longleftrightarrow \int_{\mathbb{P}(E)} \hat{\sigma}(\psi) \cdot \Omega=0
$$

Let \|\|$\psi\|\|$ be defined as follows:

$$
\begin{aligned}
\|\| \psi\|\| \equiv & \frac{k^{m}}{k \cdot k} \cdot\|\hat{\sigma}(\psi)\| \\
& +\frac{k^{m}}{k \cdot k} \cdot\left\|{ }^{M} \nabla \hat{\sigma}(\psi)\right\|+\frac{k^{m}}{k \cdot k} \cdot\left\|{ }^{M} \nabla \circ{ }^{M} \nabla \hat{\sigma}(\psi)\right\| \\
& +\frac{k^{m}}{k \cdot k} \cdot\left\|{ }^{M} \nabla \circ{ }^{M} \nabla \circ{ }^{M} \nabla \hat{\sigma}(\psi)\right\| \\
& +\frac{k^{m}}{k \cdot k} \cdot\left\|{ }^{M} \nabla \circ{ }^{M} \nabla \circ{ }^{M} \nabla \circ{ }^{M} \nabla \hat{\sigma}(\psi)\right\| \\
& +\frac{k^{m}}{k} \cdot\|\sigma(\psi)\|+\frac{k^{m}}{k} \cdot\left\|{ }^{M} \nabla \sigma(\psi)\right\| \\
& +\frac{k^{m}}{k} \cdot\left\|{ }^{M} \nabla \circ{ }^{M} \nabla \sigma(\psi)\right\| \\
& +k^{m} \cdot\left\|{ }_{[k]}^{M} \nabla \circ{ }_{[k]}^{M} \nabla \circ{ }_{[k]}^{M} \nabla \sigma(\psi)\right\| \\
& +k^{m} \cdot\left\|_{[k]}^{M} \nabla \circ{ }_{[k]}^{M} \nabla \circ{ }_{[k]}^{M} \nabla \circ{ }_{[k]}^{M} \nabla \sigma(\psi)\right\| \\
& +k^{m} \cdot\|\tilde{\sigma}(\psi)\|+\left\|{ }_{[k]} \nabla \tilde{\sigma}(\psi)\right\| \\
& +k^{m} \cdot\left\|_{[k]} \nabla \circ{ }_{[k]} \nabla \tilde{\sigma}(\psi)\right\| \\
& +k^{m} \cdot\left\|_{[k]} \nabla \circ{ }_{[k]} \nabla \circ{ }_{[k]} \nabla \tilde{\sigma}(\psi)\right\| \\
& +k^{m} \cdot \|_{[k]} \nabla \circ{ }_{[k]} \nabla \circ{ }_{[k]} \nabla \circ[k]
\end{aligned}
$$

Then there exists $k_{0} \in \mathbb{N}$ such that for $k \geq k_{0}$ we have

$$
\|\| \psi\|\| \leq C \cdot\left\|\mathbf{P}_{[k]} \psi\right\| .
$$

Here the constant $C>0$ is independent of $k \in \mathbb{N}$.

Proof. Note that

$$
\begin{aligned}
{ }^{V} \nabla \circ{ }^{V} \nabla \circ{ }^{V} \nabla \circ{ }^{V} \nabla \hat{\sigma}(\psi) & ={ }^{V} \nabla \circ{ }^{V} \nabla \circ{ }^{V} \nabla \hat{\sigma}(\psi) \\
& ={ }^{V} \nabla \circ{ }^{V} \nabla \hat{\sigma}(\psi)={ }^{V} \nabla \hat{\sigma}(\psi)=0
\end{aligned}
$$


and

$$
\begin{aligned}
\left\|{ }^{V} \nabla \circ{ }^{V} \nabla \circ{ }^{V} \nabla \circ{ }^{V} \nabla \sigma(\psi)\right\| & +\left\|^{V} \nabla \circ{ }^{V} \nabla \circ{ }^{V} \nabla \sigma(\psi)\right\| \\
& +\left\|^{V} \nabla \circ{ }^{V} \nabla \sigma(\psi)\right\|+\left\|{ }^{V} \nabla \sigma(\psi)\right\| \\
& \leq C \cdot\|\sigma(\psi)\| .
\end{aligned}
$$

These results will be used implicitly in the following derivation of (III.27). Similar results are valid for the covariant derivatives of $\hat{\sigma}(\psi)$ and $\sigma(\psi)$. Besides we will use the symbol $C_{\theta}$ to indicate a positive constant independent of $k \in \mathbb{N}$. Here $\theta \in \mathbb{N}$.

By (II.23) and (III.3) we have

$$
\|\| \hat{\sigma}(\psi)\|\| \leq C_{1} \cdot \frac{\|\mid \sigma(\psi)+\tilde{\sigma}(\psi)\| \|}{k}+C_{1} \cdot\left\|\hat{\sigma}\left(\mathbf{P}_{[k]} \psi\right)\right\| .
$$

By (II.21) and (III.1) we have

$$
\begin{aligned}
\|\| \sigma(\psi)\|\| \leq & C_{2} \cdot \frac{k^{m}}{k \cdot k} \cdot\left\|\check{Q}_{M}(\hat{\sigma}(\psi))\right\| \\
& +C_{3} \cdot \frac{\|\| \psi\|\|}{k}+C_{3} \cdot\left\|\sigma\left(\mathbf{P}_{[k]} \psi\right)\right\| \\
\leq & C_{4} \cdot\|\| \hat{\sigma}(\psi)\|\|+C_{3} \cdot \frac{\|\psi \psi\| \|}{k} \\
& +C_{3} \cdot\left\|\sigma\left(\mathbf{P}_{[k]} \psi\right)\right\| .
\end{aligned}
$$

Considering the sum

$$
2 \cdot C_{4} \cdot(\text { III.28) }+(\text { III.29) }
$$

leads to

$$
\begin{aligned}
\|\| \hat{\sigma}(\psi)\|\|+\|\| \sigma(\psi)\|\| \leq & C_{5} \cdot\left\|\hat{\sigma}\left(\mathbf{P}_{[k]} \psi\right)\right\| \\
& +C_{5} \cdot\left\|\sigma\left(\mathbf{P}_{[k]} \psi\right)\right\|+C_{5} \cdot \frac{\|\psi \psi\| \|}{k} .
\end{aligned}
$$

Now by (II.22) and (III.2) we have

$$
\begin{aligned}
\|\| \tilde{\sigma}(\psi)\|\| \leq & C_{6} \cdot \frac{k^{m}}{k} \cdot\left\|\tilde{\sigma}\left[\Delta_{V} \circ\left(-\frac{\Delta_{V} \phi_{1}}{2} \cdot \frac{\Delta_{V} \sigma(\psi)}{2}+Q_{V}\left(\sigma(\psi): \phi_{1}\right)\right)\right]\right\| \\
& +C_{7} \cdot \frac{\|\| \psi\|\|}{k}+C_{7} \cdot\left\|\tilde{\sigma}\left(\mathbf{P}_{[k]} \psi\right)\right\| \\
\text { (III.31) } & C_{8} \cdot\|\| \sigma(\psi)\|\|+C_{7} \cdot \frac{\|\psi \psi\| \|}{k}+C_{7} \cdot\left\|\tilde{\sigma}\left(\mathbf{P}_{[k]} \psi\right)\right\| .
\end{aligned}
$$

Considering the sum

$$
2 \cdot C_{8} \cdot(\text { III.30) }+(\text { III.31) }
$$


leads to

$$
\|\| \psi\|\| \leq C_{9} \cdot \frac{\|\| \psi\|\|}{k}+C_{9} \cdot\left\|\mathbf{P}_{[k]} \psi\right\| .
$$

Hence for $k \in \mathbb{N}$ satisfying $k \geq 2 \cdot C_{9}$ we obtain

$$
\|\| \psi\|\| \leq 2 \cdot C_{9} \cdot\left\|\mathbf{P}_{[k]} \psi\right\| .
$$

This completes the proof. q.e.d.

The following result can be proved by Corollary III.A and the method used in the proof of Proposition III.B.

Corollary III.B. Assume that $\psi \in \Gamma(\mathbb{P}(E): \mathbb{R})$ satisfies the normalization condition

$$
\int_{\mathbb{P}(E)} \psi \cdot \Omega=0 \Longleftrightarrow \int_{\mathbb{P}(E)} \hat{\sigma}(\psi) \cdot \Omega=0 .
$$

Then for each $\gamma \in \mathbb{N}$ there exists $k_{\gamma} \in \mathbb{N}$ such that for $k \geq k_{\gamma}$ we have

$$
\|\|\left(\Delta_{V}+\Delta_{M}\right)^{\gamma} \psi\|\| \leq C^{[\gamma]} \cdot\left\|\left(\Delta_{V}+\Delta_{M}\right)^{\gamma} \circ \mathbf{P}_{[k]} \psi\right\| .
$$

Here the constant $C^{[\gamma]}>0$ is independent of $k \in \mathbb{N}$.

Proposition III.C. Assume that $\psi \in \Gamma(\mathbb{P}(E): \mathbb{R})$ satisfies the normalization condition

$$
\int_{\mathbb{P}(E)} \psi \cdot \Omega=0 \Longleftrightarrow \int_{\mathbb{P}(E)} \hat{\sigma}(\psi) \cdot \Omega=0 .
$$

Then there exists a constant $C>0$, independent of both $N \in \mathbb{N}$ and $k \in \mathbb{N}$, such that the following statement is true: Given $N \geq 2$ there exists $k_{N} \in \mathbb{N}$ such that for $k \geq k_{N}$ we have

$$
\|\| \psi\|\| \leq C \cdot\left\|\frac{{ }_{N} \mathfrak{S}_{[k]}}{\Omega} \psi\right\| .
$$

Proof. By (II.12) we have

$$
\frac{{ }_{N} \mathfrak{S}_{[k]}}{\Omega} \psi=\mathbf{P}_{[k]} \psi+\frac{k^{m}}{k \cdot k} \cdot{ }^{4} D_{V}^{\emptyset}\left(k^{-0}\right) \psi+\frac{k^{m}}{k \cdot k \cdot k} \cdot{ }^{4} D^{\emptyset}\left(k^{-0}\right) \psi .
$$

Thus from (III.27) it follows that for $k$ large enough,

$$
\begin{aligned}
\|\| \psi\|\| \leq & C \cdot\left\|\frac{{ }_{N} \mathfrak{S}_{[k]}}{\Omega} \psi\right\|+C \cdot \frac{k^{m}}{k \cdot k} \cdot\left\|{ }^{4} D_{V}^{\emptyset}\left(k^{-0}\right) \psi\right\| \\
& +C \cdot \frac{k^{m}}{k \cdot k \cdot k} \cdot\left\|{ }^{4} D^{\emptyset}\left(k^{-0}\right) \psi\right\| \\
\leq & C \cdot\left\|\frac{\mathfrak{S}_{[k]}}{\Omega} \psi\right\|+{ }_{N} C \cdot \frac{\|\| \psi\|\|}{k} .
\end{aligned}
$$


Here the constant ${ }_{N} C>0$ depends on $N$ but is independent of $k$. Thence (III.35) is true when $k$ is sufficiently large. q.e.d.

The following result can be proved by Corollary III.B and the method used in the proof of Proposition III.C.

Corollary III.C. Assume that $\psi \in \Gamma(\mathbb{P}(E): \mathbb{R})$ satisfies the normalization condition

$$
\int_{\mathbb{P}(E)} \psi \cdot \Omega=0 \Longleftrightarrow \int_{\mathbb{P}(E)} \hat{\sigma}(\psi) \cdot \Omega=0 .
$$

Then for each $\gamma \in \mathbb{N}$ there exists a corresponding constant $C^{[\gamma]}>0$, independent of both $N \in \mathbb{N}$ and $k \in \mathbb{N}$, such that the following statement is true: Given $N \geq 2$ there exists $k_{(\gamma: N)} \in \mathbb{N}$ such that for $k \geq k_{(\gamma: N)}$ we have

$$
\|\|\left(\Delta_{V}+\Delta_{M}\right)^{\gamma} \psi\|\| \leq C^{[\gamma]} \cdot\left\|\left(\Delta_{V}+\Delta_{M}\right)^{\gamma} \circ \frac{{ }_{N} \mathfrak{S}_{[k]}}{\Omega} \psi\right\| .
$$

\section{Proof of Theorem B via the Contraction Mapping Theorem}

Assume that $U$ is a coordinate open subset of $\mathbb{P}(E)$. One nonlinear partial differential operator $\mathfrak{G}$ of polynomial form with smooth coefficients on $U$ will be called "genuinely nonlinear" if and only if for any - $\in \Gamma(U: \mathbb{R})$ each single term of $\mathfrak{G} \bullet$ can be expressed as the product of one smooth function on $U$ and the product of at least two partial derivatives of $\bullet$. Thus any nonlinear partial differential operator of polynomial form on $U$, without the zero-th order part, can be expressed as the direct sum of one linear partial differential operator on $U$ and one genuinely nonlinear partial differential operator on $U$.

Assume that $\psi$ is a smooth function on $\mathbb{P}(E)$ satisfying the normalization condition

$$
\int_{\mathbb{P}(E)} \psi \cdot \Omega=0 \Longleftrightarrow \int_{\mathbb{P}(E)} \hat{\sigma}(\psi) \cdot \Omega=0 .
$$

Let

$$
{ }_{N} \omega_{[k]}<\psi>\equiv{ }_{N} \omega_{[k]}+i \bar{\partial} \partial \psi
$$


Here ${ }_{N} \omega_{[k]} \equiv{ }_{o} \omega_{[k]}+i \bar{\partial} \partial \phi_{0}+\sum_{\theta=1}^{N} \frac{i \bar{\partial} \partial \phi_{\theta}}{k^{\theta}}$ is determined by the Induction Scheme introduced in [9]. We set $\left.{ }_{N} H_{[k]}<\psi\right\rangle$ to be the Kähler metric on $\mathbb{P}(E)$ induced by ${ }_{N} \omega_{[k]}\langle\psi\rangle$. (Here $k$ is supposed to be sufficiently large.) Let

$$
\begin{aligned}
\mathcal{S}_{[k]}\left({ }_{N} \omega_{[k]}<\psi>\right) \equiv & \frac{\left({ }_{N} \omega_{[k]}<\psi>\right)^{(-2+m+n)}}{(-2+m+n) !} \\
& \wedge\left(-c_{k} \cdot{ }_{N} \omega_{[k]}<\psi>+\frac{i}{2 \pi} \bar{\partial} \partial \log \operatorname{det}{ }_{N} H_{[k]}<\psi>\right) .
\end{aligned}
$$

Then the constant Hermitian scalar curvature equation for ${ }_{N} \omega_{[k]}<\psi>$ is

$$
\mathcal{S}_{[k]}\left({ }_{N} \omega_{[k]}<\psi>\right)=0 .
$$

Note that

$$
\mathcal{S}_{[k]}\left({ }_{N} \omega_{[k]}<\psi>\right)=\mathcal{S}_{[k]}\left({ }_{N} \omega_{[k]}\right)+\frac{{ }_{N} \mathfrak{S}_{[k]}(\psi)}{(-2+m+n) !}+{ }_{N} \mathfrak{G}_{[k]}(\psi),
$$

in which $\frac{{ }_{N} \mathfrak{S}_{[k]}(\psi)}{(-2+m+n) !}$ and ${ }_{N} \mathfrak{G}_{[k]}(\psi)$ are respectively the linear part and the "essentially genuinely nonlinear" part. Elementary Calculus shows that, on each coordinate open subset $U$ of $\mathbb{P}(E)$, the "essentially genuinely nonlinear" partial differential operator $\frac{{ }^{\mathfrak{G}}[k]}{\Omega}$ can be expressed in the following form

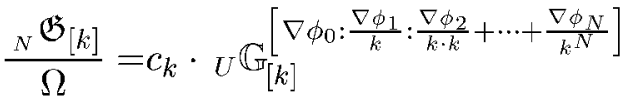

$$
\begin{aligned}
& +\frac{U \check{\widetilde{G}}_{[k]}^{\left[\nabla \phi_{0}: \frac{\nabla \phi_{1}}{k}: \frac{\nabla \phi_{2}}{k \cdot k}+\cdots+\frac{\nabla \phi_{N}}{k^{N}}\right]}}{\operatorname{det}_{N} H_{[k]}\langle\psi\rangle}
\end{aligned}
$$

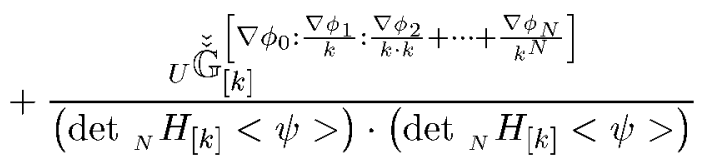

with the following property being satisfied:

$$
\text { - }{ }_{U} \mathbb{G}_{[k]}^{\left[\nabla \phi_{0}: \frac{\nabla \phi_{1}}{k}: \frac{\nabla \phi_{2}}{k \cdot k}+\cdots+\frac{\nabla \phi_{N}}{k^{N}}\right]} \text { and all the numerators on the right- }
$$

hand side of (IV.2) are 4-th order genuinely nonlinear partial differential operators of polynomial form on $U$ with coefficients depending on $k$ and on the partial derivatives of elements of the following set

$$
\left\{\phi_{0}: \frac{\phi_{1}}{k}: \frac{\phi_{2}}{k \cdot k}+\cdots+\frac{\phi_{N}}{k^{N}}\right\}
$$


in the polynomial way.

Now we consider ${ }_{U} \mathbb{G}_{[k]}^{\left[\nabla \phi_{0}: \frac{\nabla \phi_{1}}{k}: \frac{\nabla \phi_{2}}{k \cdot k}+\cdots+\frac{\nabla \phi_{N}}{k^{N}}\right]}$ as one functional in the following set of variables

$$
(\nabla \psi: \nabla \circ \nabla \psi: \nabla \circ \nabla \circ \nabla \psi: \nabla \circ \nabla \circ \nabla \circ \nabla \psi) .
$$

Let $B$ be any given compact subset of $U$. Then by the Sobolev Embedding Theorem and elementary Calculus (Chain Rule and Leibniz Rule) it is easy to see that there exists $\hat{\gamma}_{0} \in \mathbb{N}$ such that, for $\gamma \geq \hat{\gamma}_{0}$,

$$
{ }_{U} \mathbb{G}_{[k]}^{\left[\nabla \phi_{0}: \frac{\nabla \phi_{1}}{k}: \frac{\nabla \phi_{2}}{k \cdot k}+\cdots+\frac{\nabla \phi_{N}}{k^{N}}\right]}(\nabla \psi: \nabla \circ \nabla \psi: \nabla \circ \nabla \circ \nabla \psi: \nabla \circ \nabla \circ \nabla \circ \nabla \psi) \in H^{[2 \gamma]}
$$

for any $\psi \in H^{[2 \gamma+4]}$ with support on $B \subset U$. Similarly by the Sobolev Embedding Theorem and elementary Calculus (Chain Rule and Leibniz Rule) it is easy to see that there exists $\check{\gamma}_{o} \in \mathbb{N}$ such that, for $\gamma \geq \check{\gamma}_{o}$, we have

$$
\frac{{ }_{U}\left[\nabla \phi_{[k]}: \frac{\nabla \phi_{1}}{k}: \frac{\nabla \phi_{2}}{k \cdot k}+\cdots+\frac{\nabla \phi_{N}}{k^{N}}\right]}{(\nabla \psi: \nabla \circ \nabla \psi: \nabla \circ \nabla \circ \nabla \psi: \nabla \circ \nabla \circ \nabla \circ \nabla \psi)} \in H^{[2 \gamma]}
$$

and

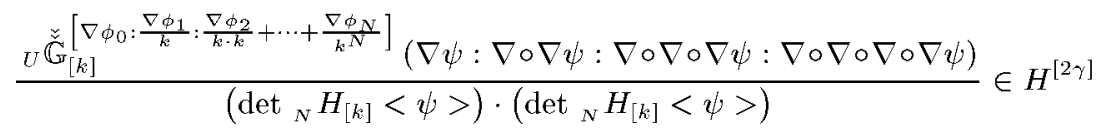

for any $\psi \in H^{[2 \gamma+4]}$ with small $H^{[2 \gamma+4]}$-norm and with support on $B \subset$ $U$. (Here we certainly require that $\check{\gamma}_{o}$ is large enough so that det ${ }_{N} H_{[k]}<$ $\psi>$, in the denominators above, is at least continuous and bounded away from 0 on $U$ for any $\psi \in H^{\left[2 \check{\gamma}_{o}+4\right]}$ with small $H^{\left[2 \check{\gamma}_{o}+4\right]}$-norm and with support on $B \subset U$.) It is important to note that $\hat{\gamma}_{o}$ and $\check{\gamma}_{o}$ are essentially independent of the choice of $(k: N)$ because we will only consider the case where both $k$ and $N$ are large.

Let ${ }_{N} \mathfrak{R}_{[k]}$ denote the inverse of $\frac{\mathfrak{S}_{[k]}}{\Omega}$. By the definition of ${ }_{N} \mathfrak{S}_{[k]}$, (II.1), it is obvious that

$$
\int_{\mathbb{P}(E)} \frac{{ }_{N} \mathfrak{S}_{[k]}(\psi)}{\Omega} \cdot \Omega=0,
$$

$\forall \psi \in \Gamma(\mathbb{P}(E): \mathbb{R})$ satisfying the normalization condition $\int_{\mathbb{P}(E)} \psi \cdot \Omega=0$

Thus for any $\gamma \in \mathbb{N}$ we have

$$
\int_{\mathbb{P}(E)} N^{\Re_{[k]}}(\psi) \cdot \Omega=0
$$


and, by Corollary III.C,

$$
\left\|{ }_{N} \mathfrak{R}_{[k]}(\varphi)\right\|_{H^{[2 \gamma+4]}} \leq C^{[\gamma]} \cdot \frac{k \cdot k}{k^{m}} \cdot\|\varphi\|_{H^{[2 \gamma]}}
$$

$\forall \varphi \in H^{[2 \gamma]}$ satisfying the normalization condition $\int_{\mathbb{P}(E)} \varphi \cdot \Omega=0$. Besides we note that the "essentially genuinely nonlinear" part $\frac{{ }_{N} \mathfrak{G}_{[k]}(\psi)}{\Omega}$ satisfies

$$
\int_{\mathbb{P}(E)} \frac{{ }_{N} \mathfrak{G}_{[k]}(\psi)}{\Omega} \cdot \Omega=0
$$

because

$$
\int_{\mathbb{P}(E)} \mathcal{S}_{[k]}\left({ }_{N} \omega_{[k]}\right)=0=\int_{\mathbb{P}(E)} \mathcal{S}_{[k]}\left({ }_{N} \omega_{[k]}<\psi>\right)
$$

by cohomological consideration.

Now for $(\gamma: q: k) \in \mathbb{N} \times \mathbb{N} \times \mathbb{N}$ we set

$$
{ }_{q} \mathbf{S}_{[k]}^{[\gamma]} \equiv\left\{\psi \in H^{[2 \gamma+4]}: \int_{\mathbb{P}(E)} \psi \cdot \Omega=0 \text { and }\|\psi\|_{H^{[2 \gamma+4]}} \leq k^{-q}\right\} .
$$

Let $\gamma_{o} \equiv \max \left(\hat{\gamma}_{o}: \check{\gamma}_{o}\right)$. Since all the "essentially genuinely nonlinear" partial differential operators $\frac{{ }_{N}^{\mathfrak{G}_{[k]}}}{\Omega}$, depending on $N$ and on $k$, have the same "essentially genuinely nonlinear" form it is easy to see that, for each $\gamma \geq \gamma_{o}$, there exists $q_{\gamma}>0$ such that the following statement is true: Given $q \geq q_{\gamma}$ and $N \geq 2$ there exists one corresponding constant $k_{(\gamma: q: N)} \in \mathbb{N}$ such that the composition map

$$
(-2+m+n) ! \cdot{ }_{N} \mathfrak{R}_{[k]} \circ \frac{{ }_{N} \mathfrak{G}_{[k]}}{\Omega}:{ }_{q} \mathbf{S}_{[k]}^{[\gamma]} \longrightarrow{ }_{q} \mathbf{S}_{[k]}^{[\gamma]}
$$

is contractive with contraction constant $\leq \frac{1}{2}$ for any $k \geq k_{(\gamma: q: N)}$.

We are now ready to solve the constant Hermitian scalar curvature equation $\mathcal{S}_{[k]}\left({ }_{N} \omega_{[k]}<\psi>\right)=0$ :

$$
\frac{\mathcal{S}_{[k]}\left({ }_{N} \omega_{[k]}\right)}{\Omega}+\frac{{ }_{N} \mathfrak{S}_{[k]}(\psi)}{(-2+m+n) ! \cdot \Omega}+\frac{{ }_{N} \mathfrak{G}_{[k]}(\psi)}{\Omega}=0 .
$$

Applying $(-2+m+n) ! \cdot{ }_{N} \Re_{[k]}$ to both sides of (IV.8) we obtain

(IV.9) $\psi+(-2+m+n) ! \cdot\left({ }_{N} \mathfrak{R}_{[k]} \circ \frac{\mathcal{S}_{[k]}\left({ }_{N} \omega_{[k]}\right)}{\Omega}+{ }_{N} \Re_{[k]} \circ \frac{{ }_{N} \mathfrak{B}_{[k]}(\psi)}{\Omega}\right)=0$. 
Now we want to apply the Contraction Mapping Theorem to (IV.9) for $\psi \in{ }_{q_{\gamma_{0}}} \mathbf{S}_{[k]}^{\left[\gamma_{0}\right]}$. Thus we must require that

$$
\left\|(-2+m+n) ! \cdot{ }_{N} \mathfrak{R}_{[k]} \circ \frac{\mathcal{S}_{[k]}\left({ }_{N} \omega_{[k]}\right)}{\Omega}\right\|_{H^{\left[2 \gamma_{o}+4\right]}}
$$

is sufficiently small when compared with $k^{-q_{\gamma_{o}}}$. However, by Corollary A of [9] and by (IV.4) (Corollary III.C), we can achieve this easily by choosing $N \in \mathbb{N}$ sufficiently large. Now we fix one such $N$. Then it is obvious that (IV.9) can be solved uniquely whenever $k$ is sufficiently large: there exists $k_{\left(\gamma_{o}: q_{\gamma_{0}}: N\right)} \in \mathbb{N}$ such that (IV.9) can be solved uniquely in ${ }_{q_{\gamma_{o}}} \mathbf{S}_{[k]}^{\left[\gamma_{o}\right]}$ for each $k \geq k_{\left(\gamma_{o}: q_{\gamma_{o}}: N\right)}$ by the Contraction Mapping Theorem.

Note that this method can be used to prove the required existence result for any choice $(\gamma: q)$ satisfying $\gamma \geq \gamma_{o}$ and $q \geq q_{\gamma}$. Thus Theorem $\mathrm{B}$ is proved. q.e.d.

\section{Appendix}

In this Appendix we prove certain results which have been used in [9] and the former context of this paper.

Proof of Proposition A of [9]. To show that

$$
\frac{-4 \pi \cdot H \cdot \sum q_{\alpha \gamma} \cdot q_{\gamma \beta} \cdot w_{\alpha} \cdot \bar{w}_{\beta}+(4 \pi+2 \pi n) \cdot q \cdot q}{H \cdot H}
$$

on $\mathbb{P}\left(\mathbb{C}^{n}\right)$ is orthogonal to the eigen-space of the lowest non-zero eigenvalue $4 \pi n$ of the (Fubini-Study) Laplacian on $\mathbb{P}\left(\mathbb{C}^{n}\right)$ we only need to prove that

$$
\text { (A.A) } \frac{-4 \pi \cdot H \cdot \sum q_{\alpha \gamma} \cdot q_{\gamma \beta} \cdot w_{\alpha} \cdot \bar{w}_{\beta}+(4 \pi+2 \pi n) \cdot q \cdot q}{H \cdot H}=\frac{\left(-4 \pi n \cdot i d .+\Delta_{F-S}\right)}{2}\left(\frac{q}{H} \cdot \frac{q}{H}\right) .
$$

We can check this easily at the point $[(0: \cdots: 0: 1)] \in \mathbb{P}\left(\mathbb{C}^{n}\right):$ Direct computation shows that at $[(0: \cdots: 0: 1)] \in \mathbb{P}\left(\mathbb{C}^{n}\right)$ we have

$$
\frac{\partial \partial}{\partial \bar{w}_{\gamma} \partial w_{\gamma}}\left(\frac{q}{H} \cdot \frac{q}{H}\right)=-2 \cdot q_{n n} \cdot q_{n n} \cdot \delta_{\gamma \gamma}+2 \cdot q_{n \gamma} \cdot q_{\gamma n}+2 \cdot q_{n n} \cdot q_{\gamma \gamma}
$$

$\forall \gamma \neq n$. Since at $[(0: \cdots: 0: 1)] \in \mathbb{P}\left(\mathbb{C}^{n}\right)$

$$
\Delta_{F-S}=-4 \pi \cdot \sum_{\gamma \neq n} \frac{\partial \partial}{\partial \bar{w}_{\gamma} \partial w_{\gamma}}
$$


we have

$$
\begin{aligned}
\left(-4 \pi n \cdot i d .+\Delta_{F-S}\right)\left(\frac{q}{H} \cdot \frac{q}{H}\right)= & -4 \pi \cdot\left[-n \cdot q_{n n} \cdot q_{n n}+2 \cdot \sum_{\gamma \neq n} q_{n \gamma} \cdot q_{\gamma n}\right] \\
= & -8 \pi \cdot\left(\sum q_{n \gamma} \cdot q_{\gamma n}\right) \\
& +(4 \pi n+8 \pi) \cdot q_{n n} \cdot q_{n n}
\end{aligned}
$$

at $[(0: \cdots: 0: 1)] \in \mathbb{P}\left(\mathbb{C}^{n}\right)$ because $q$ is traceless: $\sum q_{\gamma \gamma}=0$. Thus (A.A) is valid at $[(0: \cdots: 0: 1)] \in \mathbb{P}\left(\mathbb{C}^{n}\right)$.

But this result actually suffices to show that (A.A) is valid everywhere on $\mathbb{P}\left(\mathbb{C}^{n}\right)$ : Any point on $\mathbb{P}\left(\mathbb{C}^{n}\right)$ can be transformed to

$$
[(0: \cdots: 0: 1)] \in \mathbb{P}\left(\mathbb{C}^{n}\right)
$$

via some unitary transformation of $\mathbb{C}^{n}$. Since $\Delta_{F-S}$ commutes with unitary transformations of $\mathbb{C}^{n}$, it can be checked directly that (A.A) is valid everywhere on $\mathbb{P}\left(\mathbb{C}^{n}\right)$ by considering the action of unitary transformations of $\mathbb{C}^{n}$ on $q$. q.e.d.

Explicit expression for elements of $\mathcal{H}$. Assume that $U$ is one coordinate open subset of $M$ so that $U$ can be holomorphically identified with some open neighborhood of 0 in $\mathbb{C}^{m}$. Given one holomorphic framing

$$
\left\{s_{\gamma} \in \Gamma_{h o l}(U: E): \gamma \in \mathbb{N} \text { and } \gamma \leq n\right\}
$$

of $E$ over $U$ the bundle $\left.E\right|_{U}$ can be identified with $\mathbb{C}^{n} \times U$ holomorphically. Thus the projection map $\pi$ can be expressed explicitly as follows:

$$
\pi: \mathbb{C}^{n} \times U \ni(w: z) \longmapsto z \in U .
$$

Besides the Einstein-Hermitian connection $A$ on $E$ can now be interpreted as one $\mathfrak{g l}\left(\mathbb{C}^{n}\right)$-valued one-form on $U$ :

$$
\nabla s=s A \text {. }
$$

Let

$$
h_{\alpha \beta} \equiv H_{E}\left(s_{\alpha}: s_{\beta}\right) .
$$

We want to express elements of the distribution $\mathcal{H}$ of horizontal spaces on $\mathbb{P}(E)$ induced by the Einstein-Hermitian connection $A$ on $E$ explicitly. Suppose that $z \in U$ and $x \in T_{z}(U)$. Then for $w \neq 0$ the 
$\mathbb{C}^{n}$-directional component of the horizontal lifting of $x \in T_{z}(U)$ at $(w: z) \in \mathbb{C}^{n} \times U$ is

$$
\sum\left(\sum-w_{\gamma} \cdot A_{\theta \gamma}(\mathrm{x})\right) \cdot e_{\theta}
$$

Here $e_{\theta}$ is the $\theta$-th coordinate vector of $\mathbb{C}^{n}$. Suppose that $\left\langle\sum w_{\theta} \cdot e_{\theta}\right\rangle$ is the one-dimensional subspace of $\mathbb{C}^{n}$ spanned by the element $\sum w_{\theta} \cdot e_{\theta} \neq 0$ in $\mathbb{C}^{n}$. Then $\frac{\mathbb{C}^{n}}{\left\langle\sum w_{\theta} \cdot e_{\theta}\right\rangle}$ is the quotient space which makes the following sequence

$$
0 \longrightarrow<\sum w_{\theta} \cdot e_{\theta}>\longrightarrow \mathbb{C}^{n} \longrightarrow \frac{\mathbb{C}^{n}}{<\sum w_{\theta} \cdot e_{\theta}>} \longrightarrow 0
$$

exact. Thus for the holomorphic projection map

$$
\check{\pi}: \mathbb{P}(E) \longrightarrow M
$$

the $\mathbb{C}$-linear map from $\left\langle\sum w_{\theta} \cdot e_{\theta}\right\rangle$ to $\frac{\mathbb{C}^{n}}{\left\langle\sum w_{\theta} \cdot e_{\theta}\right\rangle}$ defined by

$$
\sum w_{\theta} \cdot e_{\theta} \longmapsto\left[\sum\left(\sum-w_{\gamma} \cdot A_{\theta \gamma}(\mathrm{x})\right) \cdot e_{\theta}\right] \in \frac{\mathbb{C}^{n}}{\left\langle\sum w_{\theta} \cdot e_{\theta}\right\rangle}
$$

is the $\mathbb{P}\left(\mathbb{C}^{n}\right)$-directional component of the horizontal lifting of $x \in T_{z}(U)$ at the point $\left(\left[\sum w_{\theta} \cdot e_{\theta}\right]: z\right) \in \mathbb{P}\left(\mathbb{C}^{n}\right) \times U$.

Special coordinate system. Now we assume that

$$
h_{\alpha \beta}=\delta_{\alpha \beta}
$$

and

$$
A=0
$$

at the point $(0: \cdots: 0) \in U$. Besides we assume that

$$
\omega_{M}\left(\frac{\partial}{\partial z_{\mu}}:-i \cdot \frac{\partial}{\partial \bar{z}_{\nu}}\right)=\delta_{\mu \nu}
$$

and

$$
d\left(\omega_{M}\left(\frac{\partial}{\partial z_{\mu}}:-i \cdot \frac{\partial}{\partial \bar{z}_{\nu}}\right)\right)=0
$$

at the point $0 \in U$. The following map

$$
\mathbb{C}^{(-1+n)} \times U \ni(\check{w}: z) \longmapsto\left(\left[\left(\check{w}: w_{n} \equiv 1\right)\right]: z\right) \in \mathbb{P}\left(\mathbb{C}^{n}\right) \times U
$$

defines one holomorphic coordinate system on the corresponding open subset of $\mathbb{P}\left(\mathbb{C}^{n}\right) \times U$. Elements of $\mathcal{H}$ can then be expressed explicitly as 
follows: Given $z \in U$ and $x \in T_{z}(U)$ the $\mathbb{C}^{(-1+n)}$-directional component of the horizontal lifting of $x \in T_{z}(U)$ at

$$
([(0: \cdots: 0: 1)]: z) \in \mathbb{P}\left(\mathbb{C}^{n}\right) \times U
$$

is

(A.C) $\sum_{\theta \neq n}-\left[\Re\left(A_{\theta n}(\mathrm{x})\right) \cdot \frac{\partial}{\partial u_{\theta}}+\Im\left(A_{\theta n}(\mathrm{x})\right) \cdot \frac{\partial}{\partial v_{\theta}}\right] \in T_{0}\left(\mathbb{C}^{(-1+n)}\right)$.

Here $\Re(\bullet)$ and $\Im(\bullet)$ are respectively the real part and the imaginary part of $\bullet$. Besides $u_{\theta}$ and $v_{\theta}$ are the $\mathbb{R}$-valued coordinate functions on $\mathbb{C}^{(-1+n)}$ :

$$
\check{w}_{\theta}=u_{\theta}+i \cdot v_{\theta} .
$$

Assume that $x_{\mu}$ and $y_{\mu}$ are the $\mathbb{R}$-valued coordinate functions on $U$ :

$$
z_{\mu}=x_{\mu}+y_{\mu}
$$

Then for each $f \in \Gamma(\mathbb{P}(E): \mathbb{R})$ we have the following result: Given $z \in U$ we have

$$
\partial_{V} f=\sum_{\gamma \neq n} \frac{\partial f}{\partial w_{\gamma}} \cdot d w_{\gamma}+\sum\left(\sum_{\theta \neq n} \frac{\partial f}{\partial w_{\theta}} \cdot A_{\theta n}\left(\frac{\partial}{\partial z_{\mu}}\right)\right) \cdot d z_{\mu}
$$

and

$$
\partial_{M} f=\sum \frac{\partial f}{\partial z_{\mu}} \cdot d z_{\mu}+\sum\left(\sum_{\theta \neq n}-\frac{\partial f}{\partial w_{\theta}} \cdot A_{\theta n}\left(\frac{\partial}{\partial z_{\mu}}\right)\right) \cdot d z_{\mu}
$$

at the point $([(0: \cdots: 0: 1)]: z) \in \mathbb{P}\left(\mathbb{C}^{n}\right) \times U$. (Note that, in these formulae, we have adopted the convention that $w_{\gamma}=\check{w}_{\gamma}, \forall \gamma \neq n$, and $w_{n} \equiv 1$.) Besides we note that

$$
\partial_{V} f=\sum_{\gamma \neq n} \frac{\partial f}{\partial w_{\gamma}} \cdot d w_{\gamma}
$$

and

$$
\partial_{M} f=\sum \frac{\partial f}{\partial z_{\mu}} \cdot d z_{\mu}
$$

at any point $([(\breve{w}: 1)]: 0) \in \mathbb{P}\left(\mathbb{C}^{n}\right) \times U$. Now for the representative

$$
\frac{i}{2 \pi} \cdot \bar{\partial} \partial \log H_{L^{*}}
$$


of the Euler class $e\left(L^{*}\right)$ of $L^{*}$ on $\mathbb{P}(E)$ induced by the Einstein-Hermitian metric $H_{E}$ on $E$ we have the following result:

$$
\begin{aligned}
& \frac{i}{2 \pi} \cdot \bar{\partial} \partial \log H_{L^{*}} \\
& =\frac{-i}{2 \pi} \cdot \sum_{\alpha \neq n \neq \beta}\left[\frac{-\left(\sum h_{\alpha \sigma} \cdot \bar{w}_{\sigma}\right) \cdot \sum h_{\tau \beta} \cdot w_{\tau}}{\left(\sum h_{\alpha \beta} \cdot w_{\alpha} \cdot \bar{w}_{\beta}\right)^{2}}+\frac{h_{\alpha \beta}}{\sum h_{\alpha \beta} \cdot w_{\alpha} \cdot \bar{w}_{\beta}}\right] \cdot d \bar{w}_{\beta} \wedge d w_{\alpha} \\
& +\frac{-i}{2 \pi} \cdot \sum_{\beta \neq n}\left[\frac{-\left(\sum \frac{\partial h_{\sigma \tau}}{\partial z_{\mu}} \cdot w_{\sigma} \cdot \bar{w}_{\tau}\right) \cdot \sum h_{\alpha \beta} \cdot w_{\alpha}}{\left(\sum h_{\alpha \beta} \cdot w_{\alpha} \cdot \bar{w}_{\beta}\right)^{2}}+\frac{\sum \frac{\partial h_{\alpha \beta}}{\partial z_{\mu}} \cdot w_{\alpha}}{\sum h_{\alpha \beta} \cdot w_{\alpha} \cdot \bar{w}_{\beta}}\right] \cdot d \bar{w}_{\beta} \wedge d z_{\mu}
\end{aligned}
$$

$$
\begin{aligned}
& +\frac{-i}{2 \pi} \cdot \sum_{\alpha \neq n}\left[\frac{-\left(\sum \frac{\partial h_{\sigma \tau}}{\partial z_{\nu}} \cdot w_{\sigma} \cdot \bar{w}_{\tau}\right) \cdot \sum h_{\alpha \beta} \cdot \bar{w}_{\beta}}{\left(\sum h_{\alpha \beta} \cdot w_{\alpha} \cdot \bar{w}_{\beta}\right)^{2}}+\frac{\sum \frac{\partial h_{\alpha \beta}}{\partial \bar{z}_{\nu}} \cdot \bar{w}_{\beta}}{\sum h_{\alpha \beta} \cdot w_{\alpha} \cdot \bar{w}_{\beta}}\right] \cdot d \bar{z}_{\nu} \wedge d w_{\alpha} \\
& +\frac{-i}{2 \pi} \cdot \sum\left[\frac{-\left(\sum \frac{\partial h_{\alpha \beta}}{\partial z_{\mu}} \cdot w_{\alpha} \cdot \bar{w}_{\beta}\right) \cdot\left(\sum \frac{\partial h_{\sigma \tau}}{\partial \bar{z}_{\nu}} \cdot w_{\sigma} \cdot \bar{w}_{\tau}\right)}{\left(\sum h_{\alpha \beta} \cdot w_{\alpha} \cdot \bar{w}_{\beta}\right)^{2}}\right] \cdot d \bar{z}_{\nu} \wedge d z_{\mu} \\
& +\frac{-i}{2 \pi} \cdot \sum\left[\frac{\left(\sum \frac{\partial h_{\alpha \beta}}{\partial \bar{z}_{\nu} \partial z_{\mu}} \cdot w_{\alpha} \cdot \bar{w}_{\beta}\right)}{\sum h_{\alpha \beta} \cdot w_{\alpha} \cdot \bar{w}_{\beta}}\right] \cdot d \bar{z}_{\nu} \wedge d z_{\mu} .
\end{aligned}
$$

We will now use the Special Coordinate System to prove the unproved results stated in Section I.

Proposition I.A. Given $f \in \Gamma(\mathbb{P}(E): \mathbb{R})$ we have the following equalities

$$
i \cdot \Lambda_{V} \circ(\bar{\partial} \partial)_{V} f=\frac{\Delta_{V} f}{2}
$$

and

$$
i \cdot \Lambda_{M} \circ(\bar{\partial} \partial)_{M} f=\frac{\Delta_{M} f}{2}
$$

Proof. We have

$$
\Delta_{V}=d_{V}^{*} \circ d_{V}=d^{*} \circ d_{V}=d^{*} \circ \partial_{V}+d^{*} \circ \bar{\partial}_{V}
$$

and

$$
\Delta_{M}=d_{M}^{*} \circ d_{M}=d^{*} \circ d_{M}=d^{*} \circ \partial_{M}+d^{*} \circ \bar{\partial}_{M} .
$$

Once we have proved that

$$
d^{*} \circ \partial_{V}=i \cdot \Lambda_{V} \circ(\bar{\partial} \partial)_{V}
$$


and

$$
d^{*} \circ \partial_{M}=i \cdot \Lambda_{M} \circ(\bar{\partial} \partial)_{M}
$$

our assertion follows immediately because

$$
d^{*} \circ \bar{\partial}_{V}=\overline{d^{*} \circ \partial_{V}}=\overline{i \cdot \Lambda_{V} \circ(\bar{\partial} \partial)_{V}}=i \cdot \Lambda_{V} \circ(\bar{\partial} \partial)_{V}
$$

and

$$
d^{*} \circ \bar{\partial}_{M}=\overline{d^{*} \circ \partial_{M}}=\overline{i \cdot \Lambda_{M} \circ(\bar{\partial} \partial)_{M}}=i \cdot \Lambda_{M} \circ(\bar{\partial} \partial)_{M} .
$$

Now we prove that (A.1) and (A.2) are valid at the point

$$
([(0: \cdots:: 1)]: 0) \in \mathbb{P}\left(\mathbb{C}^{n}\right) \times U
$$

using the Special Coordinate System. Note that

$$
\begin{aligned}
d^{*}=-* d *= & \sum_{\gamma \neq n}-\left(d w _ { \gamma } \left\llcorner\nabla_{\frac{\partial}{\partial w_{\gamma}}}+d \bar{w}_{\gamma}\left\llcorner\nabla_{\frac{\partial}{\partial \bar{w}_{\gamma}}}\right)\right.\right. \\
& +\sum\left(d z _ { \mu } \left\llcorner\nabla_{\frac{\partial}{\partial z_{\mu}}}+d \bar{z}_{\mu}\left\llcorner\nabla_{\frac{\partial}{\partial \bar{z}_{\mu}}}\right)\right.\right.
\end{aligned}
$$

at the point $([(0: \cdots: 0: 1)]: 0) \in \mathbb{P}\left(\mathbb{C}^{n}\right) \times U$. Here $\bullet\llcorner\bullet$ is the $\mathbb{C}$ linear inner product acting on the complexified cotangent bundle of $\mathbb{P}(E)$ induced by the Riemannian metric on $\mathbb{P}(E)$. Thus at the point $([(0: \cdots: 0: 1)]: 0) \in \mathbb{P}\left(\mathbb{C}^{n}\right) \times U$ we have

$$
\begin{aligned}
Y d^{*} \circ \partial_{V} f & -\sum_{\alpha \neq n \text { and } \beta \neq n} \frac{\partial f}{\partial w_{\alpha}} \cdot\left[d w _ { \beta } \left\llcorner\left(\nabla_{\frac{\partial}{\partial w_{\beta}}} d w_{\alpha}\right)+d \bar{w}_{\beta}\left\llcorner\left(\nabla_{\frac{\partial}{\partial w_{\beta}}} d w_{\alpha}\right)\right]\right.\right. \\
& -\sum_{\gamma \neq n} \frac{\partial f}{\partial w_{\gamma}} \cdot \sum\left[d z _ { \mu } \left\llcorner\nabla_{\frac{\partial}{\partial z_{\mu}}} d w_{\gamma}+d \bar{z}_{\mu}\left\llcorner\nabla_{\frac{\partial}{\partial \bar{z}_{\mu}}} d w_{\gamma}\right]\right.\right. \\
& -\sum_{\gamma \neq n} \frac{\partial \partial f}{\partial \bar{w}_{\gamma} \partial w_{\gamma}} \cdot 2 \pi+ \\
& -\sum_{\theta \neq n} \frac{\partial f}{\partial w_{\theta}} \cdot\left[\sum \frac{\partial}{\partial \bar{z}_{\mu}} A_{\theta n}\left(\frac{\partial}{\partial z_{\mu}}\right)\right]
\end{aligned}
$$


and

$$
\begin{aligned}
d^{*} \circ \partial_{M} f= & -\sum \frac{\partial f}{\partial z_{\mu}} \cdot \sum_{\gamma \neq n}\left[d w _ { \gamma } \left\llcorner\left(\nabla_{\frac{\partial}{\partial w_{\gamma}}} d z_{\mu}\right)+d \bar{w}_{\gamma}\left\llcorner\left(\nabla_{\frac{\partial}{\partial \bar{w}_{\gamma}}} d z_{\mu}\right)\right]\right.\right. \\
& -\sum \frac{\partial f}{\partial z_{\mu}} \cdot \sum\left[d z _ { \nu } \left\llcorner\left(\nabla_{\frac{\partial}{\partial z_{\nu}}} d z_{\mu}\right)+d \bar{z}_{\nu}\left\llcorner\left(\nabla_{\frac{\partial}{\partial \bar{z}_{\nu}}} d z_{\mu}\right)\right]\right.\right. \\
& -\sum \frac{\partial \partial f}{\partial \bar{z}_{\mu} \partial z_{\mu}} \\
& -\sum_{\theta \neq n} \frac{\partial f}{\partial w_{\theta}} \cdot\left[\sum-\frac{\partial}{\partial \bar{z}_{\mu}} A_{\theta n}\left(\frac{\partial}{\partial z_{\mu}}\right)\right] .
\end{aligned}
$$

Using (A.C) and (A.D) in the defining formula of the Levi-Civita connection induced by the Hermitian form (metric)

$$
\check{\omega}=\Pi_{\mathcal{C}_{V}}\left(\frac{i}{2 \pi} F_{H_{L^{*}}}\right)+\check{\pi}^{*} \omega_{M}
$$

on $\mathbb{P}(E)$ it can be checked directly that

$$
d^{*} \circ \partial_{V} f=-2 \pi \cdot \sum_{\gamma \neq n} \frac{\partial \partial f}{\partial \bar{w}_{\gamma} \partial w_{\gamma}}+\sum_{\theta \neq n} \frac{\partial f}{\partial w_{\theta}} \cdot\left[\sum-\frac{\partial}{\partial \bar{z}_{\mu}} A_{\theta n}\left(\frac{\partial}{\partial z_{\mu}}\right)\right]
$$

and

$$
d^{*} \circ \partial_{M} f=-\sum \frac{\partial \partial f}{\partial \bar{z}_{\mu} \partial z_{\mu}}+\sum_{\theta \neq n} \frac{\partial f}{\partial w_{\theta}} \cdot\left[\sum \frac{\partial}{\partial \bar{z}_{\mu}} A_{\theta n}\left(\frac{\partial}{\partial z_{\mu}}\right)\right]
$$

at $([(0: \cdots: 0: 1)]: 0) \in \mathbb{P}\left(\mathbb{C}^{n}\right) \times U$. Since $A$ is Einstein-Hermitian we have for $\theta \neq n$

$$
\sum \frac{\partial}{\partial \bar{z}_{\mu}} A_{\theta n}\left(\frac{\partial}{\partial z_{\mu}}\right)=0
$$

at $0 \in U$. Thus $d^{*} \circ \partial_{V} f=i \cdot \Lambda_{V} \circ(\bar{\partial} \partial)_{V} f$ and $d^{*} \circ \partial_{M} f=i \cdot \Lambda_{M} \circ(\bar{\partial} \partial)_{M} f$ at the point $([(0: \cdots: 0: 1)]: 0) \in \mathbb{P}\left(\mathbb{C}^{n}\right) \times U$ and thence everywhere on $\mathbb{P}(E)$. q.e.d.

\section{Proposition I.B.}

$$
\Delta_{M} \circ \Delta_{V}=\Delta_{V} \circ \Delta_{M}
$$

Proof. Assume that $f \in \Gamma(\mathbb{P}(E): \mathbb{R})$. By Proposition I.A we have

$$
\frac{\Delta_{V} f}{2} \cdot \frac{e\left(L^{*}\right)^{(-1+n)} \wedge \omega_{M}^{m}}{(-1+n) ! \cdot m !}=i \bar{\partial} \partial f \wedge \frac{e\left(L^{*}\right)^{(-2+n)} \wedge \omega_{M I}^{m}}{(-2+n) ! \cdot m !}
$$


and

$$
\frac{\Delta_{M} f}{2} \cdot \frac{e\left(L^{*}\right)^{(-1+n)} \wedge \omega_{M}^{m}}{(-1+n) ! \cdot m !}=i \bar{\partial} \partial f \wedge \frac{e\left(L^{*}\right)^{(-1+n)} \wedge \omega_{M}^{(-1+m)}}{(-1+n) ! \cdot(-1+m) !} .
$$

Now we use the Special Coordinate System to show that

$$
\Delta_{M} \circ \Delta_{V}=\Delta_{V} \circ \Delta_{M}
$$

at the point $([(0: \cdots: 0: 1)]: 0) \in \mathbb{P}\left(\mathbb{C}^{n}\right) \times U$. Using (A.D) and (A.3) it is easy to see that

$$
\begin{aligned}
\frac{\mathcal{L}_{\frac{\partial}{\partial \bar{z} \mu_{\mu}}} \circ \mathcal{L}_{\frac{\partial}{\partial z_{\mu}}} \Delta_{V} f}{2} \cdot \frac{e\left(L^{*}\right)^{(-1+n)} \wedge \omega_{M}^{m}}{(-1+n) ! \cdot m !} \\
+\frac{\Delta_{V} f}{2} \cdot \frac{e\left(L^{*}\right)^{(-2+n)} \wedge\left(\mathcal{L}_{\frac{\partial}{\partial \bar{z}_{\mu}}} \circ \mathcal{L}_{\frac{\partial}{\partial z_{\mu}}} e\left(L^{*}\right)\right) \wedge \omega_{M}^{m}}{(-2+n) ! \cdot m !} \\
=\frac{\left(\mathcal{L}_{\frac{\partial}{\partial \bar{z}_{\mu}}} \circ \mathcal{L}_{\frac{\partial}{\partial z_{\mu}}} i \bar{\partial} \partial f\right) \wedge e\left(L^{*}\right)^{(-2+n)} \wedge \omega_{M}^{m}}{(-2+n) ! \cdot m !} \\
+\frac{i \bar{\partial} \partial f \wedge e\left(L^{*}\right)^{(-3+n)} \wedge\left(\mathcal{L}_{\frac{\partial}{\partial \bar{z}_{\mu}}} \circ \mathcal{L} \frac{\partial}{\partial z_{\mu}} e\left(L^{*}\right)\right) \wedge \omega_{M}^{m}}{(-3+n) ! \cdot m !}
\end{aligned}
$$

by applying $\mathcal{L}_{\frac{\partial}{\partial \bar{z}_{\mu}}} \circ \mathcal{L}_{\frac{\partial}{\partial z_{\mu}}}$ to both sides of (A.3). Now by (A.D) we have

$$
\begin{aligned}
& \Pi_{\mathcal{C}_{V}} \circ\left(\sum \mathcal{L}_{\frac{\partial}{\partial \bar{z}_{\mu}}} \circ \mathcal{L}_{\frac{\partial}{\partial z_{\mu}}} e\left(L^{*}\right)\right)= \frac{i}{2 \pi} \cdot \sum_{\alpha \neq n \text { and } \beta \neq n} d \bar{w}_{\beta} \wedge d w_{\alpha} \\
& \cdot\left[\sum\left(-\frac{\partial \partial h_{\alpha \beta}}{\partial \bar{z}_{\mu} \partial z_{\mu}}+h_{\alpha \beta} \cdot \frac{\partial \partial h_{n n}}{\partial \bar{z}_{\mu} \partial z_{\mu}}\right)\right] \\
&=0,
\end{aligned}
$$

because $H_{E}$ is Einstein-Hermitian. Thus we infer from (A.5)

$$
\begin{aligned}
-\frac{\Delta_{M}}{2}( & \left.\frac{\Delta_{V} f}{2}\right) \cdot \frac{e\left(L^{*}\right)^{(-1+n)} \wedge \omega_{M}^{m}}{(-1+n) ! \cdot m !} \\
& =\frac{\sum \mathcal{L}_{\frac{\partial}{\partial \bar{z}_{\mu}}} \circ \mathcal{L}_{\frac{\partial}{\partial z_{\mu}}} \Delta_{V} f}{2} \cdot \frac{e\left(L^{*}\right)^{(-1+n)} \wedge \omega_{M}^{m}}{(-1+n) ! \cdot m !} \\
& =\left(\sum \mathcal{L}_{\frac{\partial}{\partial z_{\mu}}} \circ \mathcal{L}_{\frac{\partial}{\partial z_{\mu}}} i \bar{\partial} \partial f\right) \wedge \frac{e\left(L^{*}\right)^{(-2+n)} \wedge \omega_{M I}^{m}}{(-2+n) ! \cdot m !}
\end{aligned}
$$


at the point $([(0: \cdots: 0: 1)]: 0) \in \mathbb{P}\left(\mathbb{C}^{n}\right) \times U$. Similarly it is easy to see that

$$
\begin{aligned}
& \left(2 \pi \cdot \sum_{\gamma \neq n} \frac{\mathcal{L}_{\frac{\partial}{\partial \bar{w}_{\gamma}}} \circ \mathcal{L}_{\frac{\partial}{\partial w_{\gamma}} \Delta_{M} f}}{2}\right) \cdot \frac{e\left(L^{*}\right)^{(-1+n)} \wedge \omega_{M}^{m}}{(-1+n) ! \cdot m !} \\
& =\frac{e\left(L^{*}\right)^{(-1+n)} \wedge \omega_{M}^{(-1+m)}}{(-1+n) ! \cdot(-1+m) !} \wedge\left(2 \pi \cdot \sum_{\gamma \neq n} \mathcal{L}_{\frac{\partial}{\partial \bar{w}_{\gamma}}} \circ \mathcal{L}_{\frac{\partial}{\partial w_{\gamma}}} i \bar{\partial} \partial f\right)
\end{aligned}
$$

by applying

$$
2 \pi \cdot \sum_{\gamma \neq n} \mathcal{L}_{\frac{\partial}{\partial \bar{w}_{\gamma}}} \circ \mathcal{L}_{\frac{\partial}{\partial w_{\gamma}}}
$$

to both sides of (A.4) and using the following result:

$\Pi_{\mathcal{C}_{V}} \circ\left(2 \pi \cdot \sum_{\gamma \neq n} \mathcal{L}_{\frac{\partial}{\partial \bar{w}_{\gamma}}} \circ \mathcal{L}_{\frac{\partial}{\partial w_{\gamma}}} e\left(L^{*}\right)\right)=i \cdot \sum_{\alpha \neq n \text { and } \beta \neq n} d \bar{w}_{\beta} \wedge d w_{\alpha}$

$$
\begin{aligned}
& \cdot\left[\sum_{\gamma \neq n}\left(h_{\alpha \gamma} \cdot h_{\gamma \beta}+h_{\alpha \beta} \cdot h_{\gamma \gamma}\right)\right] \\
= & i \cdot n \cdot \sum_{\gamma \neq n} d \bar{w}_{\gamma} \wedge d w_{\gamma} \\
= & -2 \pi \cdot n \cdot \Pi_{\mathcal{C}_{V}} \circ e\left(L^{*}\right)
\end{aligned}
$$

at the point $([(0: \cdots: 0: 1)]: 0) \in \mathbb{P}\left(\mathbb{C}^{n}\right) \times U$. Now we infer from (A.7) and (A.6) that

$$
\begin{aligned}
-\frac{\Delta_{V}}{2} & \left(\frac{\Delta_{M} f}{2}\right) \cdot \frac{e\left(L^{*}\right)^{(-1+n)}}{(-1+n) ! \cdot m !} \\
& =\frac{e\left(L^{*}\right)^{(-1+n)}}{(-1+n) ! \cdot(-1+m) !} \wedge\left(2 \pi \cdot \sum_{\gamma \neq n}^{(-1+m)} \mathcal{L}_{\frac{\partial}{\partial \bar{w} \gamma}} \circ \mathcal{L}_{\frac{\partial}{\partial w_{\gamma}}} i \bar{\partial} \partial f\right) \\
& =\frac{e\left(L^{*}\right)^{(-2+n)} \wedge \omega_{M}^{m}}{(-2+n) ! \cdot m !} \wedge\left(\sum \mathcal{L}_{\frac{\partial}{\partial \bar{z}_{\mu}}} \circ \mathcal{L}_{\frac{\partial}{\partial z_{\mu}}} i \bar{\partial} \partial f\right) \\
& =-\frac{\Delta_{M}}{2}\left(\frac{\Delta_{V} f}{2}\right) \cdot \frac{e\left(L^{*}\right)^{(-1+n)} \wedge \omega_{M}^{m}}{(-1+n) ! \cdot m !}
\end{aligned}
$$

at the point $([(0: \cdots: 0: 1)]: 0) \in \mathbb{P}\left(\mathbb{C}^{n}\right) \times U$. Thus $\Delta_{M} \circ \Delta_{V}=$ $\Delta_{V} \circ \Delta_{M}$ at the point $([(0: \cdots: 0: 1)]: 0) \in \mathbb{P}\left(\mathbb{C}^{n}\right) \times U$ and thence everywhere on $\mathbb{P}(E)$. q.e.d. 
Proof of Lemma I.A. By using the Special Coordinate System we only need to show that

$$
\frac{\Delta_{V} Q_{M}(\hat{\sigma}(\psi))}{4 \pi}=\check{Q}_{M}(\hat{\sigma}(\psi))
$$

at $([(0: \cdots: 0: 1)]: 0) \in \mathbb{P}\left(\mathbb{C}^{n}\right) \times U$. Actually it is easy to see that

$$
\begin{aligned}
&-\frac{\Delta_{V} Q_{M}(\hat{\sigma}(\psi))}{2} \cdot e\left(L^{*}\right)^{(-1+n)} \wedge \frac{\omega_{M}^{m}}{m !} \\
&=e\left(L^{*}\right)^{(-1+n)} \wedge \frac{\omega_{M}^{(-2+m)}}{(-2+m) !} \wedge i(\bar{\partial} \partial)_{M} \hat{\sigma}(\psi) \wedge 2 \pi \\
& \cdot \sum_{\gamma \neq n} \mathcal{L}_{\frac{\partial}{\partial \bar{w}_{\gamma}}} \circ \mathcal{L}_{\frac{\partial}{\partial w_{\gamma}}} \Pi_{\mathcal{C}_{M}} e\left(L^{*}\right)
\end{aligned}
$$

by applying

$$
2 \pi \cdot \sum_{\gamma \neq n} \mathcal{L}_{\frac{\partial}{\partial \bar{w}_{\gamma}}} \circ \mathcal{L}_{\frac{\partial}{\partial w_{\gamma}}}
$$

to both sides of

$$
\begin{aligned}
Q_{M}(\hat{\sigma}(\psi)) \cdot e\left(L^{*}\right)^{(-1+n)} \wedge \frac{\omega_{M}^{m}}{m !} \\
=e\left(L^{*}\right)^{(-1+n)} \wedge \frac{\omega_{M}^{(-2+m)}}{(-2+m) !} \wedge i(\bar{\partial} \partial)_{M} \hat{\sigma}(\psi) \\
\quad \wedge\left(i(\bar{\partial} \partial)_{M} \phi_{0}+\Pi_{\mathcal{C}_{M}} e\left(L^{*}\right)\right)
\end{aligned}
$$

and using (A.E). However by (A.D) it is easy to see that

$$
\begin{aligned}
2 \pi \cdot \sum_{\gamma \neq n} \mathcal{L}_{\frac{\partial}{\partial \bar{w}_{\gamma}}} \circ \mathcal{L}_{\frac{\partial}{\partial w_{\gamma}}} \Pi_{\mathcal{C}_{M}} e\left(L^{*}\right)=i \cdot \sum d \bar{z}_{\nu} \wedge d z_{\mu} \\
\cdot\left[-\sum_{\gamma \neq n} \frac{\partial \partial h_{\gamma \gamma}}{\partial \bar{z}_{\nu} \partial z_{\mu}}+\sum_{\gamma \neq n} h_{\gamma \gamma} \cdot \frac{\partial \partial h_{n n}}{\partial \bar{z}_{\nu} \partial z_{\mu}}\right] \\
=-2 \pi \cdot\left(\operatorname{trace}\left(\frac{i}{2 \pi} F_{H_{E}}\right)+n \cdot \Pi_{\mathcal{C}_{M}} e\left(L^{*}\right)\right)
\end{aligned}
$$

at the point $([(0: \cdots: 0: 1)]: 0) \in \mathbb{P}\left(\mathbb{C}^{n}\right) \times U$. Thus

$$
\frac{\Delta_{V} Q_{M}(\hat{\sigma}(\psi))}{4 \pi}=\check{Q}_{M}(\hat{\sigma}(\psi))
$$

follows immediately.

To see that $\triangle_{V} Q_{M}(\hat{\sigma}(\psi)) \in \Gamma(M: W)$ we simply consider the action of unitary transformations of $\mathbb{C}^{n}$ on the expression of

$$
\left(\operatorname{trace}\left(\frac{i}{2 \pi} F_{H_{E}}\right)+n \cdot \Pi_{\mathcal{C}_{M}} e\left(L^{*}\right)\right)
$$

in Special Coordinate Systems. q.e.d. 


\section{References}

[1] D. Burns \& P. de Bartolomeis, Stability of vector bundles and extremal metrics, Invent. Math. 92 (1988) 403-407.

[2] S. Bando \& T. Mabuchi, Uniqueness of Einstein-Kähler metrics modulo connected group actions, Algebraic Geometry, Sendai, 1985, Adv. Stud. Pure Math. 10 Kinokuniya, Tokyo and North-Holland, Amsterdam, 1987.

[3] E. Calabi, Extremal Kähler metrics, Seminar on Differential Geometry, Ann. of Math. Stud., Princeton University Press, New Jersey, 102 (1982).

[4] S. K. Donaldson, Anti-self-dual Yang-Mills connections on complex algebraic surfaces and stable vector bundles, Proc. London Math. Soc. 50 (1985) 1-26.

[5] Infinite determinants, stable bundles and curvature, Duke Math. J. 54 (1987) 231-247.

[6] $\underset{1997 .}{ }$, Remarks on Gauge theory, complex geometry and 4-manifold topology, 1997.

[7] D. Gilbarg \& N. S. Trudinger, Elliptic partial differential equations of second order, (Second Edition) Springer, Berlin, 1983.

[8] P. Griffiths \& J. Harris, Principles of algebraic geometry, John Wiley, N.Y., 1978.

[9] Y-J Hong, Ruled manifolds with constant Hermitian scalar curvature, Math. Res. Letters 5 (1998) 657-673.

[10] S. Kobayashi, Differential geometry of complex vector bundles, Iwanami Shoten and Princeton Univ. Press, 1987.

[11] C. LeBrun \& S. R. Simanca, Extremal Kähler metrics and complex deformation theory, Geom. and Funct. Anal. 4 (1994) 298-336.

[12] C. LeBrun, Polarized 4-manifolds, extremal Kähler metrics, and Seiberg-Witten theory, Math. Res. Letters 2 (1995) 653-662.

[13] M. Lübke, Stability of Einstein-Hermitian vector bundles, Manuscripta Math. 42 (1983) $245-257$.

[14] C. H. Taubes, The Seiberg-Witten invariants and symplectic forms, Math. Res. Letters 1 (194) 809-822.

[15] - The Seiberg-Witten invariants and the Gromov invariants, Math. Res. Letters 2 (1995) 221-238.

[16] G. Tian, Kähler-Einstein metrics with positive scalar curvature, Invent. Math. 137 (1997) 1-37. 
[17] K. Uhlenbeck \& S-T Yau, On the existence of Hermitian Yang-Mills connections in stable bundles, Comm. on Pure and Appl. Math., Supplement 39 (1986) S257S293.

[18] S-T Yau, On the Ricci curvature of a compact Kähler manifold and the complex Monge-Ampere equation. I, Comm. on Pure and Appl. Math. 31 (1978) 339-411.

Transworld Institute of Technology, Taiwan 